\title{
DNA-based molecular force sensors in cytoskeletal networks and cells
}

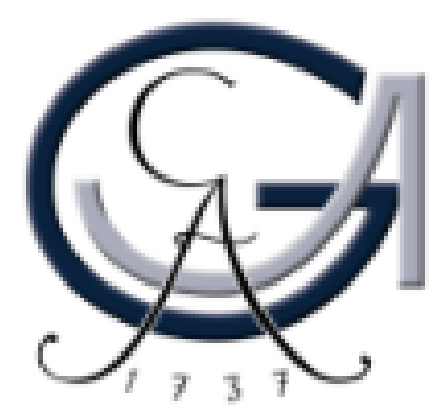

by Meenakshi Prabhune 


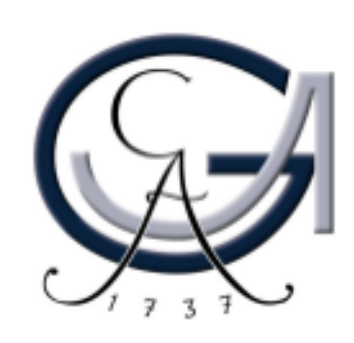

\title{
DNA-based molecular force sensors in cytoskeletal networks and cells
}

\author{
Dissertation \\ for the award of the degree \\ "Doctor rerum naturalium" \\ of the Georg-August-Universitt Göttingen
}

within the doctoral program

Physics of Biological and Complex Systems

of the Göttingen Graduate School for Neurosciences, Biophysics, and

Molecular Biosciences (GGNB)

of the Georg-August University School of Science (GAUSS)

submitted by

Meenakshi Prabhune

from Pune, India

Göttingen, June $5^{\text {th }}, 2015$ 


\section{Thesis committee}

Prof. Dr. Christoph F. Schmidt (supervisor)

Drittes Physikalisches Institut, Georg-August Universität Göttingen

Dr. Florian Rehfeldt (supervisor)

Drittes Physikalisches Institut, Georg-August Universität Göttingen

Prof. Dr. Sarah Köster

Institut für Röntgenphysik, Georg-August Universität Göttingen

Prof. Dr. Fred Wouters

Abteilung Neuro- und Sinnesphysiologie, Universitätsmedizin

Georg-August Universität Göttingen

\section{Members of the Examination Board}

Prof. Dr. Christoph F. Schmidt ( $1^{\text {st }}$ reviewer and supervisor)

Drittes Physikalisches Institut, Georg-August Universität Göttingen

Dr. Florian Rehfeldt (supervisor)

Drittes Physikalisches Institut, Georg-August Universität Göttingen

Prof. Dr. Sarah Köster (2 ${ }^{\text {nd }}$ reviewer)

Institut für Röntgenphysik, Georg-August Universität Göttingen

Prof. Dr. Fred Wouters

Abteilung Neuro- und Sinnesphysiologie, Universitätsmedizin

Georg-August Universität Göttingen

\section{Further members of the Examination Board}

Dr. Iwan Schaap

Drittes Physikalisches Institut, Georg-August Universität Göttingen

Dr. Alexander Egner

Laser-Laboratorium, Georg-August Universität Göttingen

Date of the oral examination: July $10^{\text {th }}, 2015$ 


\section{Affidavit}

I herewith declare that I have produced this dissertation without the prohibited assistance of third parties and without making use of aids other than those specified; notions taken over directly or indirectly from other sources have been identified as such. This dissertation has not previously been presented in identical or similar form to any other German or foreign examination board.

The thesis work was conducted from November 2011 to June 2015 under the supervision of Prof. Dr. Christoph F. Schmidt and Dr. Florian Rehfeldt at the Third Institute of Physics Biophysics. 


\section{Contents}

1 Introduction 9

1.1 The cell and its cytoskeleton . . . . . . . . . . . . . . . . . . . . . . . . . 10

1.2 Actin structure $\ldots \ldots \ldots \ldots \ldots$. . . . . . . . . . . . . . . . . . . . . . . . . 11

1.3 Microtubules . . . . . . . . . . . . . . . . . . . . . . . . . . . . . . . . . 12

1.4 Role of the cytoskeleton in cellular processes . . . . . . . . . . . . . . . . . . 14

1.5 Outline of thesis . . . . . . . . . . . . . . . . . . . . . . . . . . . . . 16

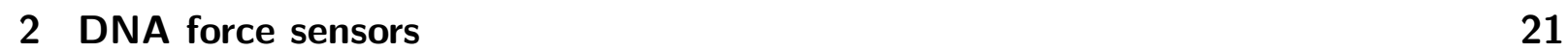

2.1 Advances in the field of force sensing . . . . . . . . . . . . . . . . . . . . . . . 22

2.2 DNA structure $\ldots \ldots \ldots \ldots$. . . . . . . . . . . . . . . . . . . . . . . . . . . . . . . . . . . . 23

2.3 Advantages of a DNA-based force sensor . . . . . . . . . . . . . . . . . . . . . . 24

2.4 Fluorescence resonance energy transfer (FRET) . . . . . . . . . . . . . . . . . . 25

2.5 Design of the DNA force sensor . . . . . . . . . . . . . . . . . . . . . . . . . . . . . . . . . . . . . . . . . . .

2.6 Immobilized force sensor experiments . . . . . . . . . . . . . . . . . . . . . . . . . . . . . . . . . . . . . . . . . . .

2.7 Force sensing in cytoskeletal networks . . . . . . . . . . . . . . . . . . . 36

3 Rheology 43

3.1 Rheology of elastic and viscous materials . . . . . . . . . . . . . . . . . . . . . 44

3.2 Linear viscoelasticity . . . . . . . . . . . . . . . . . . . . . . . . . . . . . . . . . . 46

3.3 Rheometer . . . . . . . . . . . . . . . . . . . . . . . . . . . . . . . . . . . . . . . . . 48

3.4 Mechanics of biopolymer networks . . . . . . . . . . . . . . . . . . . . . . . . 51

3.5 Crosslinking microtubules with DNA . . . . . . . . . . . . . . . . . . . . . . . . . 52

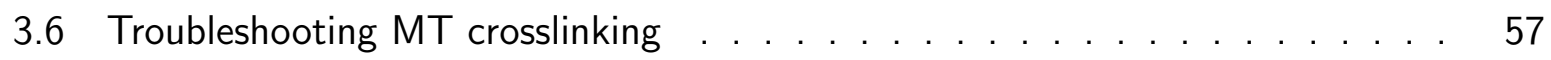

3.7 Sulfo-SMCC prevents annealing of MTs . . . . . . . . . . . . . . . . . . . . . 58

3.8 Crosslinking of MTs with biotin and neutravidin . . . . . . . . . . . . . . . 61

3.9 Actin crosslinking with Halotag . . . . . . . . . . . . . . . . . . . . . . . . 62

3.10 Possible artifacts in rheometry . . . . . . . . . . . . . . . . . . . 66

\begin{tabular}{|lll}
\hline & DNA force sensors in cells & 75
\end{tabular}

\begin{tabular}{lll}
\hline 5 & Summary and Outlook & 79
\end{tabular} 
6 Materials and methods 83

6.1 Preparation of DNA $\ldots \ldots \ldots \ldots$

$6.2 \quad$ Immobilization protocols $\ldots \ldots \ldots \ldots$. . . . . . . . . . . . . . . . 87

6.3 Preparation of microtubules $\ldots \ldots \ldots$. . . . . . . . . . . . . . . . . . . . 89

6.4 Preparation of actin $\ldots \ldots \ldots \ldots$. . . . . . . . . . . . . . . . . . . 91

6.5 Preparation of Halotag-fusion proteins $\ldots \ldots \ldots . \ldots . \ldots 93$

$6.6 \quad$ Cell culture . . . . . . . . . . . . . . . . . . . . . . . . . . . . . . . . . . . 94

\begin{tabular}{ll}
\hline Glossary & 100
\end{tabular}

\begin{tabular}{ll}
\hline Acknowledgements & 103
\end{tabular}

\begin{tabular}{ll}
\hline Curriculum Vitae & 105
\end{tabular} 


\section{List of Figures}

1.1 Schematic of a cell showing cytoskeletal filaments. . . . . . . . . . . . . . . . 10

$1.2 \quad$ Actin polymerization and crosslinking. . . . . . . . . . . . . . . . . . . . . . . 11

1.3 Microtubule polymerization and dynamic instability. . . . . . . . . . . . . . 13

1.4 Dynamic cellular processes. $\ldots \ldots \ldots \ldots$. . . . . . . . . . . . . . . . . . . 15

2.1 Structure of double-stranded DNA. . . . . . . . . . . . . . . . . . . . . . . . . . . . 23

2.2 Mechanisms of separation of complementary DNA strands. . . . . . . . . . . . . 24

2.3 Mechanism of FRET . . . . . . . . . . . . . . . . . . . . . . . . . . . . . . . . . . 25

2.4 Schematic of irreversible DNA sensor. . . . . . . . . . . . . . . . . . . . . . . . 27

2.5 Control experiments on irreversible sensor. . . . . . . . . . . . . . . . . . . . . . 28

2.6 Schematic of reversible DNA sensor. $\ldots \ldots \ldots$. . . . . . . . . . . . . . . . . . . . . . . . . . . $\quad 29$

2.7 Control experiments on a reversible DNA sensor. . . . . . . . . . . . . . . . . . . . . . . 29

2.8 Single molecule controls of DNA sensor. . . . . . . . . . . . . . . . . . . . . . 31

2.9 Immobilization of thiolated DNA on coated coverslips. . . . . . . . . . . . . . . 32

2.10 Magnetic beads to pull on immobilized DNA sensors. . . . . . . . . . . . . . . . 33

2.11 AFM experiments on immobilized DNA sensors. . . . . . . . . . . . . . . . . . . . . . . . . . . . . . . . . . . 35

2.12 Shear flow to unfold DNA hairpins. . . . . . . . . . . . . . . . . . . . . . . . . 35

2.13 Bulk shear of microtubules crosslinked with DNA sensor. . . . . . . . . . . . . 37

2.14 Microscopic shear of networks. . . . . . . . . . . . . . . . . . . . . . . . 38

2.15 Experiments with minimized bleaching of fluorophores. . . . . . . . . . . . . . 38

3.1 Rheology of elastic and viscous materials. . . . . . . . . . . . . . . . . . . . . . 45

3.2 Models for viscoelastic materials. . . . . . . . . . . . . . . . . . . . . . . 47

3.3 Rheometer and oscillatory test. . . . . . . . . . . . . . . . . . . . . . . . . . 48

3.4 Linear rheology tests. $\ldots \ldots \ldots$. . . . . . . . . . . . . . . . . . . . . . . . . . . 50

3.5 Schematic of the crosslinking protocol. . . . . . . . . . . . . . . . . . . . . . . . 53

3.6 Linear viscoelastic measurements of MT networks. . . . . . . . . . . . . . . . . 54

3.7 Differential measurements of MT networks. . . . . . . . . . . . . . . . . . . 56

3.8 Confocal images of networks. . . . . . . . . . . . . . . . . . . . . . . . . . 57

3.9 NHS ester dye labeling primary amines of MTs. . . . . . . . . . . . . . . . . . . 58

3.10 Length distribution of treated and untreated MTs after long incubation. . . . . 59

3.11 Length distributions of treated and untreated MTs after $2 \mathrm{hrs}$ and $24 \mathrm{hrs}$ incubation times. . . . . . . . . . . . . . . . . . . . 60 
3.12 Biotin-neutravidin crosslinked networks. . . . . . . . . . . . . . . . . . . . 62

3.13 Images of entangled and crosslinked actin. . . . . . . . . . . . . . . . 63

3.14 Schematic of crosslinking actin using Halotag binding strategy. . . . . . . . . . 64

3.15 Aggregation of actin with Lifeact-Halotag protein. . . . . . . . . . . . . . . . 64

3.16 Confocal and STED imaging of actin. . . . . . . . . . . . . . . . . . . 64

3.17 Gels showing Lifeact-Halotag purification and binding to actin. . . . . . . . . . 65

3.18 Critical gelation of microtubules. . . . . . . . . . . . . . . . . . 67

3.19 Volume based artifacts in the rheometer. . . . . . . . . . . . . . . . . . . 68

3.20 Stainless steel bottom plate of rheometer. . . . . . . . . . . . . . . . . . 68

3.21 Comparison of different materials of bottom plates of the rheometer. . . . . . 69

3.22 Increase in $G^{\prime}$ over time. . . . . . . . . . . . . . . . . . . . . . . . 70

4.1 Introducing DNA force sensor in cells. . . . . . . . . . . . . . . . . . . . . . 77

6.1 DNA strands on gels. $\ldots \ldots \ldots \ldots$

6.2 Image analysis routine. . . . . . . . . . . . . . . . . . . . . . . . . . . . . . . 91 


\section{Chapter 1}

\section{Introduction}

A cell is a highly organized, dynamic system assembled from various molecular components. The cytoskeleton, a polymer meshwork, is an essential part of the eukaryotic cell that maintains its structural integrity. The following chapter introduces the structure and functions of cytoskeletal filaments. The importance of the cytoskeleton in active processes such as cell division, migration and adhesion is also explained. The chapter ends with an outline of the thesis, briefly describing the contents of each chapter. 


\subsection{The cell and its cytoskeleton}

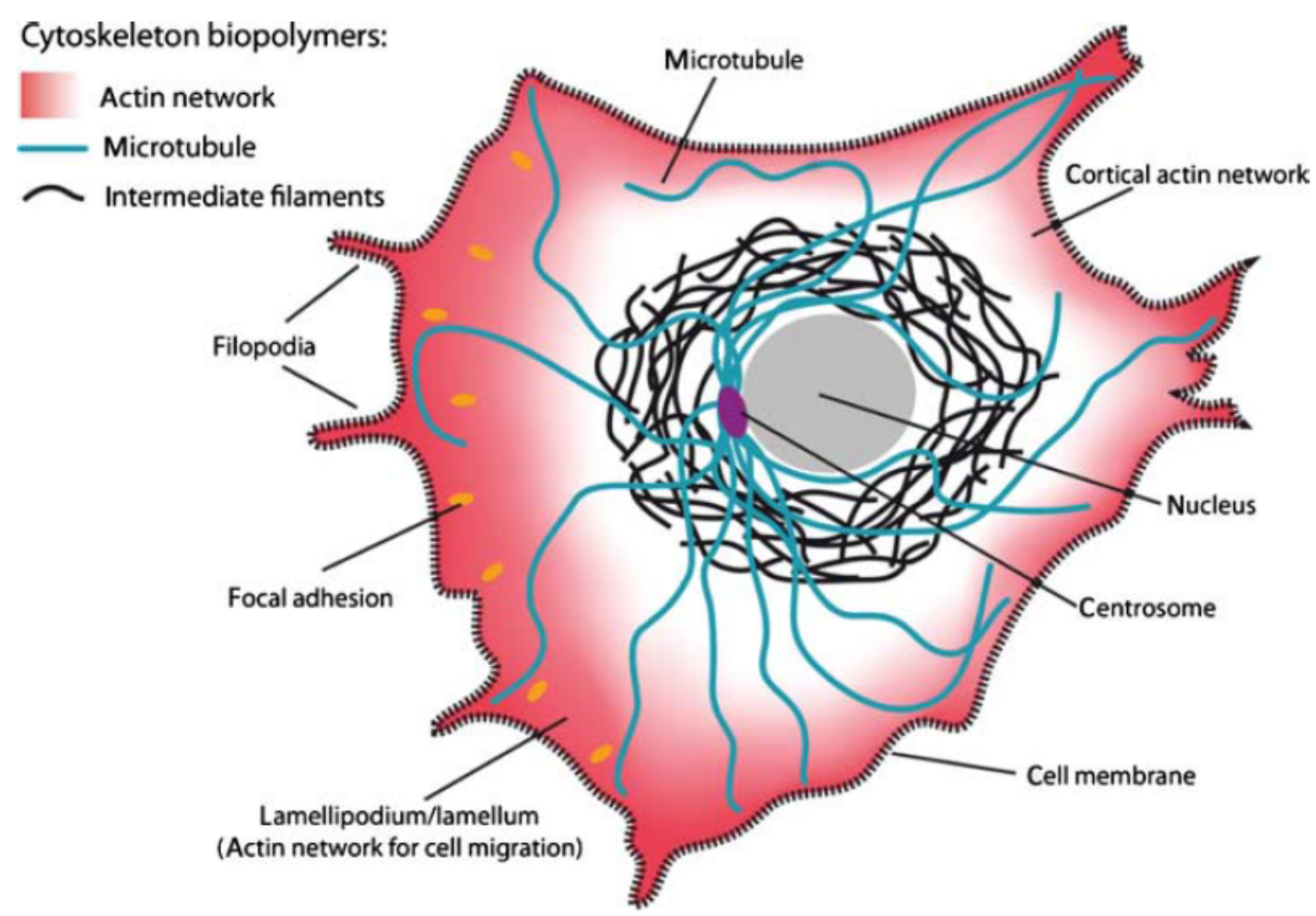

Figure 1.1: Schematic of a cell showing cytoskeletal filaments. Cells contain three types of cytoskeletal filaments - microtubules, intermediate filaments and actin. Microtubules are stiff biopolymers that span the entire cell. Intermediate filaments are commonly localized close to the cell nucleus. Actin filaments form dense networks in the cortex, close to the cell membrane. Filopodia and lamellopodia are protrusions of actin networks, important for cell adhesion and migration. Figure adapted from Ref. 1

Cells can be described as highly complex and active living matter. Eukaryotic cells comprise a scaffold of polymers, called the cytoskeleton, which is important for maintaining the structural integrity of cells. The key feature of the cytoskeleton is that it shows highly dynamic reorganization; yet maintaining order. The generation of a hierarchical complexity makes the cytoskeleton an emergent system. Emergent properties are described as macrostate properties, which arise from interactions of the underlying molecular components.

There are three classes of intracellular biopolymers - actin, microtubules (MTs) and intermediate filaments (IFs) ${ }^{2}$ (see Fig. 1.1). These cytoskeletal polymers vary in their persistence lengths over orders of magnitudes; largely differing in their mechanics and functionality. MTs are rigid polymers that are responsible for intracellular transport and cell division. Actin is a semiflexible polymer, which is crosslinked into networks and bundles. Actin plays a key role in maintaining cellular structure and in cell migration. The third type of biopolymers are the 
intermediate filaments that also contribute to the mechanical properties of living cells. All the polymer types show stochastic fluctuations in length, the origin of which lies in polymerization and depolymerization of filaments. ${ }^{3-5}$ The global impact of variability in local arrangements of cytoskeletal components is exploited in cellular functions.

The properties of cytoskeletal filaments, actin and MTs in particular, are the subject of ongoing studies. Although the functioning of intermediate filaments is comparatively less studied, their role in the complex interplay of components in cells is nontrivial. [6 However, in the following sections we shall only focus on actin and MTs to comprehend the prominent influence of these filaments on cytoskeletal dynamics.

\subsection{Actin structure}

(A)

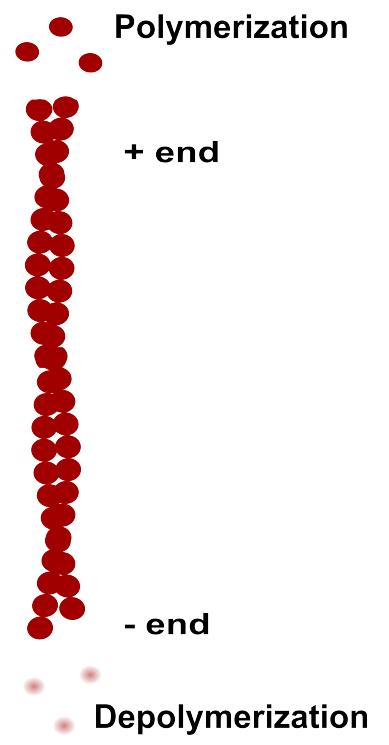

(B)

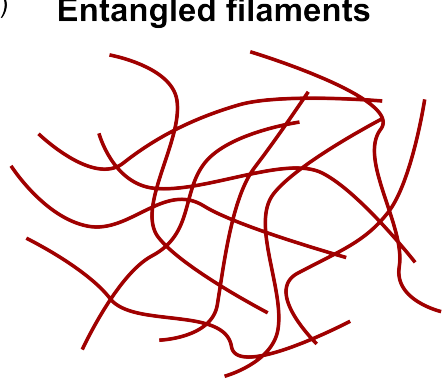

Bundled network

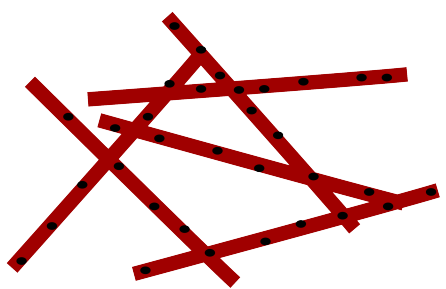

Crosslinked actin network

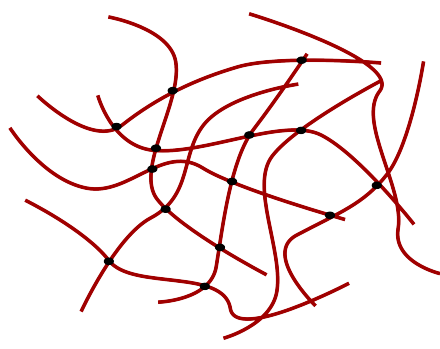

Composite network

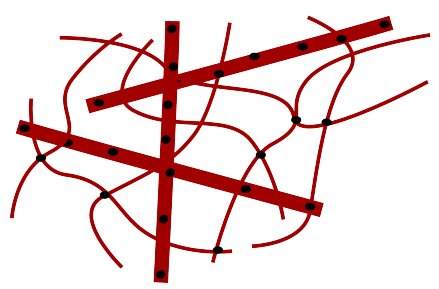

Figure 1.2: Actin polymerization and crosslinking. (A) Helical actin filaments are polymerized from G-actin monomers in presence of ATP. Hydrolysis of ATP to ADP results in asymmetrical polymerization and depolymerization kinetics at the ends of the filament. This results in a polar structure with a plus and a minus end, where monomers assemble at the plus end and travel through the filament before dissociating at minus end. This process is termed as 'treadmilling'. (B) The network architecture of a crosslinked actin network is regulated by the type and concentration of crosslinkers. Low crosslinker concentrations result in an isotropic, homogeneous network. Increasing the crosslinker concentration results in bundles of actin when the crosslinkers are small. Composite clusters of actin bundles are observed in the case of long and flexible crosslinkers.

Actin is a highly conserved protein found in abundance in eukaryotic cells. The monomeric form of actin (G-actin) has a molecular weight of $42 \mathrm{kDa}$. G-actin, on binding of adenosine 
triphosphate (ATP), polymerizes into actin filaments (F-actin) under physiological salt conditions, as shown in Fig. 1.2 A. The polymerized actin filaments are helical, with a diameter of approximately $10 \mathrm{~nm}$ and a mean length of 6-7 $\mu \mathrm{m} .7$ The arrangement of subunits in a particular orientation effectively imparts polarity to the actin filaments. ${ }^{5}$ The presence of a plus (barbed) end and minus (pointed) end results in a thermodynamically non-equilibrium state of the polymer. Asymmetrical polymerization and depolymerization kinetics at the filament ends arise from hydrolysis of ATP, which is bound to G-actin, to ADP. The ADP actin monomers have a reduced binding affinity for the neighboring monomers. Therefore, the critical concentration, which is the concentration of G-actin at which the polymerization and depolymerization rates are equal, is significantly higher at the minus-end than at the plus-end of the filament. The resultant phenomenon of 'treadmilling' refers to the traveling of newly added monomers along the filament from plus to minus end, where they dissociate. ${ }^{8}$

Mechanically, actin is a semiflexible polymer at physiological length scales, with a persistence length of approximately $10 \mu \mathrm{m}$. The self organization of actin filaments into isotropic networks, clusters or bundles is regulated by a range of crosslinking proteins called actin binding proteins (ABPs), as shown in Fig. 1.2B. The association with different ABPs largely influence the structure and integrity of actin networks. ${ }^{9}$ Functionally, actin maintains the cell shape and plays an important role in cellular process such as adhesion and migration.

\subsection{Microtubules}

Microtubules (MTs) are another type of cytoskeletal filaments, which are highly stiff (persistence length in $\mathrm{mm}$ ) tubular polymers. Microtubules play an important role in cell division and vesicle transport in cells. ${ }^{2}$ Structurally, they are hollow cylinders with a diameter of $25 \mathrm{~nm}$, assembled from molecular subunits called tubulin. The heterodimers of $\alpha$ and $\beta$ tubulin polymerize in presence of nucleotide GTP to form a protofilament.11 The head-to-tail arrangement of tubulin subunits renders polarity to the protofilaments; they consist of a plus and a minus end. Microtubules typically consist of 13 or 14 protofilaments arranged in parallel with the same polarity, ${ }^{12}$ as shown in Fig. 1.3 .

The varying polymerization and depolymerization kinetics at ends of MTs make them nonequilibrium polymers. During polymerization process, hydrolysis of the $\beta$ tubulin bound GTP to GDP reduces the binding constants, thereby destabilizing the MT. The subsequent depolymerization and shrinkage of the MT is called catastrophe. A phenomenon called 'rescue', in which GTP bearing dimers form a cap on the filament can prevent the catastrophe process. ${ }^{13}$ The rapid switching between phases of growth and shrinkage is called dynamic instability of microtubules.

These special properties of microtubules are relevant for their biological function. Polarity is essential for vesicle transport, as molecular motors traverse MTs in a specific direction. The dynamic instability of MTs is especially important in spindle formation during cell division. 
(A)

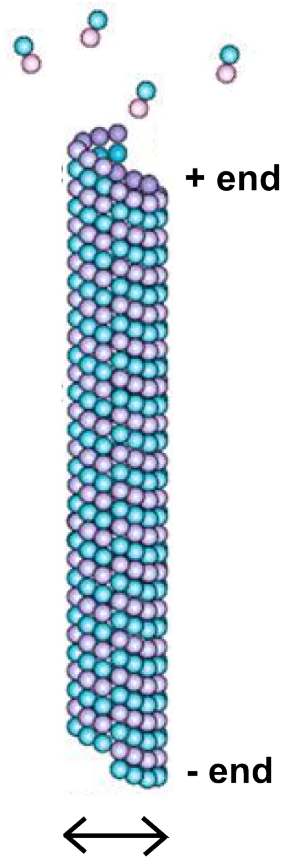

$24 \mathrm{~nm}$
(B)

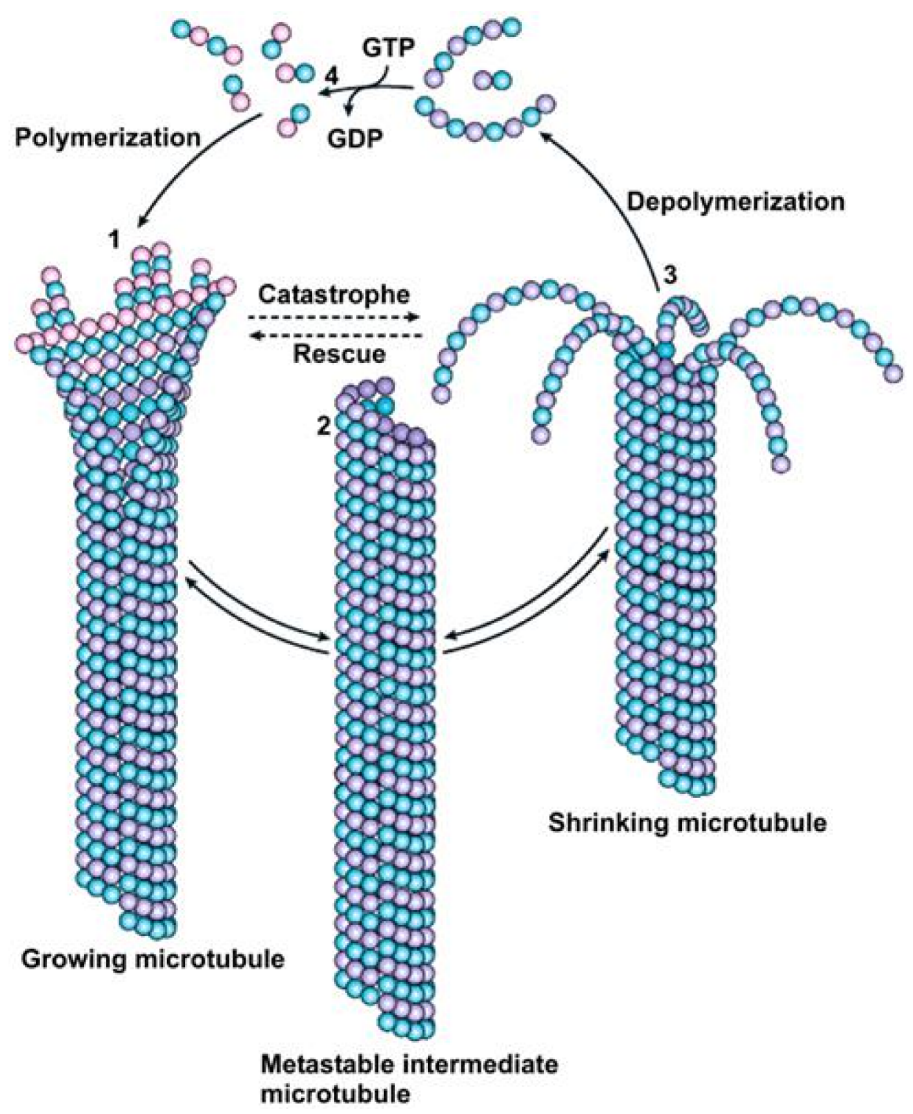

Figure 1.3: Microtubule polymerization and dynamic instability. (A) Heterodimers of $\alpha$ and $\beta$ tubulin polymerize, on binding of GTP, to form a protofilament. Typically, 13 protofilaments are arranged in parallel to form a microtubule, which is a hollow cylinder of 24 $\mathrm{nm}$ diameter and a length in tens of $\mu \mathrm{m}$. (B) During the polymerization process, the GTP, which is bound to $\beta$ tubulin, is hydrolyzed to GDP. This results in destabilization of the microtubule and subsequent depolymerization, termed 'catastrophe'. A phenomenon called 'rescue', in which GTP bearing dimers form a cap on the filament can prevent the catastrophe process. The rapid switching between phases of growth and shrinkage is called dynamic instability of microtubules. Figure adapted from Ref. 10. 


\subsection{Role of the cytoskeleton in cellular processes}

The importance of actin in cell mechanics has been demonstrated by treatment of cells with drugs that specifically target the actin cytoskeleton. Drugs such as latrunculin or cytochalasin $D$, which depolymerize actin filaments, result in a decrease of the cellular rigidity and affect cell migration. $114-17$ It was found from these experiments that the major contributor to cellular elasticity is the cortical layer of entangled and bundled actin below the membrane. ${ }^{18 \mid 19}$

Actin also plays an important role in the cellular processes of adhesion, as shown in Fig. 1.4A, B. Cells adhere to a substrate through multimolecular assemblies called focal adhesions. $\frac{2021}{21}$ Focal adhesions are the intracellular linkages between actin cytoskeleton and integrins, which further connect the cell to the extracellular matrix. They can transmit as well as respond to mechanical forces. Quantification of forces ( $\mathrm{pN}$ range) transmitted at the cell-substrate interface has been the focus of many cell adhesion studies.22-24 Filopodia, which are membrane bound extensions of tightly bundled actin filaments that protrude from the cell, are known to mediate the search for new adhesion sites of a cell to its extracellular matrix. ${ }^{25 \mid 26}$

Apart from cell adhesion, filopodia are also important for cell migration, in conjunction with another flat extended actin network protrusion, the lamellopodium. $27 / 28$ The mechanism of cellular locomotion by crawling is based on breaking cell symmetry by generating a 'front' and a 'rear' (see Fig. 1.4C). The polymerization of actin at the leading edge of the cell results in pushing forces that propel the cell forward. This is accompanied by detachment of focal adhesions at the rear end of the cell through contractile forces generated by myosin II motors. ${ }^{29}$ - 31

While actin plays a pivotal role in cell mechanics, adhesion and migration, microtubules are imperative for the process of cell division. The spindle apparatus, formed during cell division, consists of microtubules that are spatially organized and oriented by molecular motors and various microtubule binding proteins,, 3233 as shown in Fig. $1.4 \mathrm{E}, \mathrm{F}$. The mitotic spindle is responsible for the segregation of chromosomal DNA with forces in the $\mathrm{nN}$-range. ${ }^{34}$ This is achieved through a combination of two types of active forces. The first one is a pushing-pulling force arising from polymerization-depolymerization of microtubules. ${ }^{35+-37]}$ Another type of force is the sliding force generated by motors between the microtubules. $38-40$

It must be emphasized that the functionality of cytoskeletal polymers is intertwined with each other. Although the roles of actin or MTs may be individually dominant in a particular process, the overall smooth functioning of cellular processes is not devoid of interactions between the cytoskeletal filaments. For instance, cell migration is largely an actin dependent process. However, MTs are essential for motility in growth cones of neuronal cells. In fact, the interaction of MTs with actin in the peripheral region of the growth cones is crucial for path finding in the cone, $10 \mid 41 / 42$ as shown in Fig. 1.4D. On similar lines, cell division is also not a solely microtubule dependent process. Actin and myosin motors form a contractile ring at the equator of the cell that separates the two daughter cells during the last step of division ${ }^{43}$ (see Fig. $1.4 \mathrm{E}, \mathrm{F}$ ). It is therefore imperative to comprehend that there is an intricate mechanical and biochemical interplay between these biopolymers in cells. ${ }^{41}$ Although the fundamental nature of this crosstalk is not completely understood, it is clear that the cytoskeletal filaments cannot be treated as unrelated separate entities of a cell. 
(A)

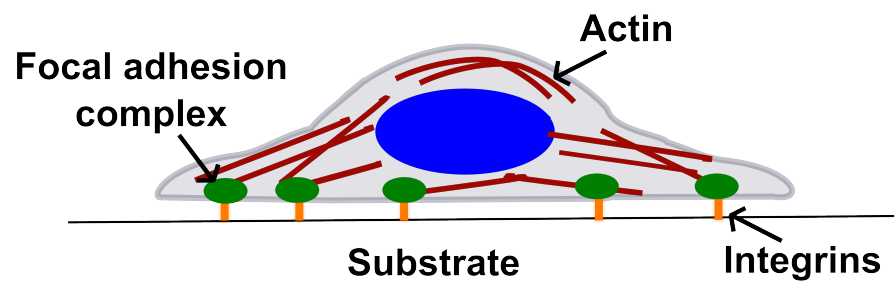

(C)

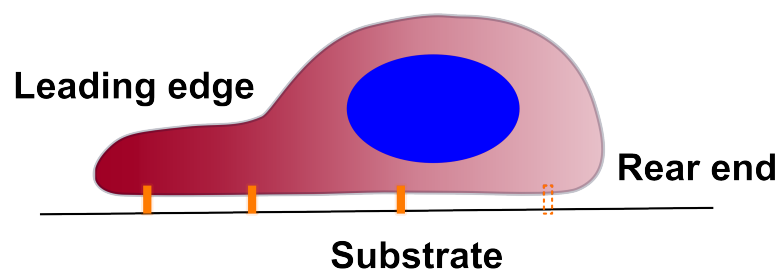

(E)

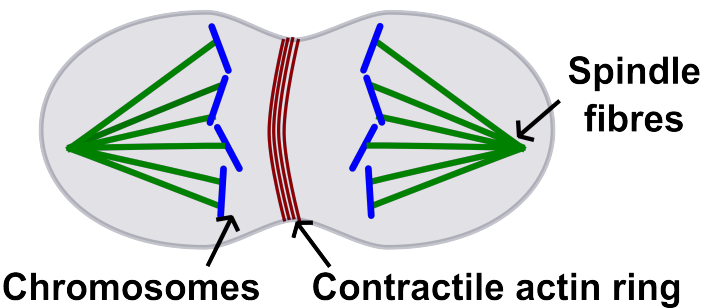

(B)

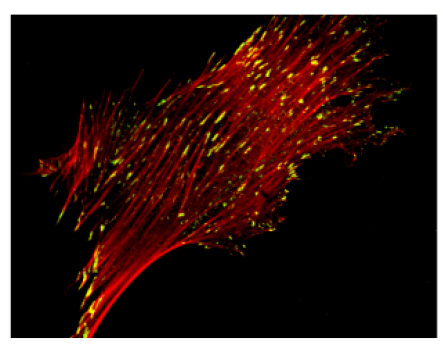

(D)
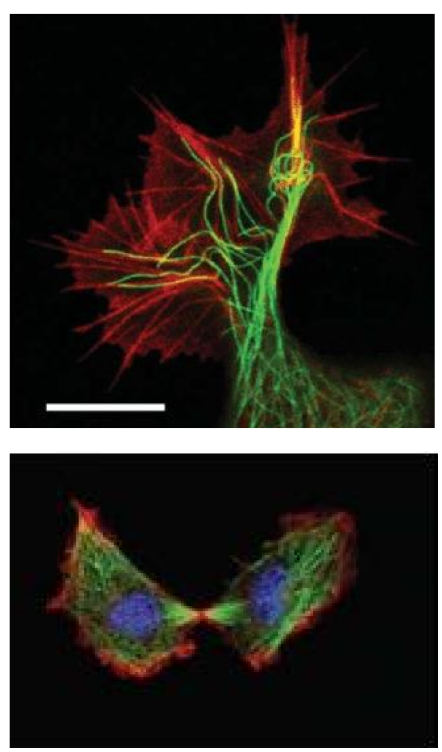

Figure 1.4: Dynamic cellular processes. (A) Schematic of a cell adhered to a 2D substrate. The actin cytoskeleton is connected to the substrate through multimolecular assemblies called focal adhesions and integrins. (B) Fluorescently labeled human mesenchymal stem cell adhering to glass showing actin cytoskeleton in red and focal adhesion contacts in green and yellow (permission Florian Rehfeldt). (C) Schematic of a crawling cell. Actin polymerizes at the leading edge, which propels the cell forward. This is accompanied by detachment of focal adhesions at rear end of the cell through contractile forces generated by myosin II. (D) NG-108 15 neuronal cell showing growth cones. Microtubules play an important role along with actin in the migration of neuronal growth cone. Actin, shown in red, forms the lamellipodium and filopodia of the cone and MTs (green) explore the periphery of the growth cone to target adhesion sites. Scale bar is $10 \mu \mathrm{m}$. Figure adapted from Ref. 1. (E) Schematic of the last phase in cell division. Spindle fibres, formed from microtubules, pull the chromosomal DNA towards the opposite poles. A contractile ring consisting of actin and myosin II motors is formed in the centre of the cell that eventually separates the daughters cells. (F) Cytokinesis in cell with actin labeled in red, microtubules in green and DNA in blue. Image adapted from Ref. 1 . 


\subsection{Outline of thesis}

The elastic properties of cells have been measured using different methods such as optical tweezers, atomic force microscopy (AFM), magnetic bead cytometry and micropipette aspiration, to name a few. $18 / 44 \mid 48$ The observed values of cellular rigidity were found to be sensitive to several parameters such as the method of measurement, geometry of cells, sample preparation and most importantly, the extracellular environment. $\frac{49}{\text { It }}$ is now known that cells respond to the stiffness of the substrate by reorganization of their actin cytoskeleton and changes in internal stresses, which in turn influences their own stiffness. ${ }^{[5]}$ A striking example are fibroblasts, which were shown to modulate their stiffness according to substrate rigidity, within a stiffness range spanning that of soft tissues. ${ }^{51}$ The most drastic and convincing case is the differentiation of stem cells into neurons, muscles or bone cells, depending on the stiffness of the substrate..$^{52}$ The implication that cell adhesion and mechanics are interdependent processes governed by the feedback loop of the cytoskeleton is crucial in understanding both the topics. This led to studies on quantification of forces exerted at the cell-substrate interface. The field of mechanosensing has made enormous progress from measuring coarse-grained cellular traction forces using beads embedded in soft substrates to advanced force-sensor designs capable of measuring forces on single molecule level. The next step is to extend the current research to include investigation of dynamic cellular processes such as migration and division in a unified fashion. So far, we lack a tool that can be commonly applied for quantifying forces in multiple cellular processes. To realize this, we have developed a DNA-based force sensor that can bind to cytoskeletal filaments in cells, sensitive to forces in range of few pN. Chapter 2 covers a detailed account of progress in the field of force sensing, followed by the design and working of the DNA force sensor.

The intricate design of processes from a plethora of biochemical components in cells can be overwhelming and complicated to reproduce. Isolated, reconstituted systems have, therefore, proven to be useful experimental systems. The number of components can be minimized for a bottom-up approach and also precisely controlled in many parameters. Investigation of reconstituted actin networks, for instance, gave a good macroscopic overview on the types of actin networks inside cells. The network architecture was shown to highly depend on the type and compliance of crosslinking proteins. ${ }^{9}$ The nonlinear mechanical properties of actin crosslinked with a compliant crosslinker in vitro closely resembled those of living cells. ${ }^{53}$ In the spirit of understanding the complex nature of cytoskeletal networks, we study mechanics of artificially crosslinked composite networks. Another advantage of this approach is that it can be easily implemented in silico, allowing us to extrapolate information about local stresses to experimental systems. Chapter 3 describes the process of assembling crosslinked composite networks and elaborates on rheological methods used for investigating the same.

The accomplishments from in vitro experiments increasingly add new pieces of the puzzle to our knowledge. However, a caveat in these systems is that they cannot fully mimic cellular response, in particular, the numerous internal feedback mechanisms in cells. How does one solve the paradoxical situations of a simpler, reductionist approach at the risk of losing valuable information against a highly complex system that proves to be difficult to comprehend? We need to find a fine balance of both approaches; understanding isolated parts of processes in 
vitro and simultaneously testing in vivo to fill the gaps. Therefore, we tested our DNA-based force sensor in in vitro cytoskeletal networks as well as inside cells. The in vitro experiments are described in Chapter 2, while the experimental trials of introducing the force sensor in human mesenchymal cells (hMSCs) are discussed in Chapter 4.

Chapter 5 includes a general discussion of the experiments and an outlook for follow-up studies.

The biochemical protocols and other methods are elaborated in Chapter 6 .

\section{Bibliography}

[1] Huber, F.; Schnauss, J.; Rönicke, S.; Rauch, P.; Müller, K.; Fütterer, C.; Käs, J. Advances in physics 2013, 62, 1-112.

[2] Alberts, B.; Bray, D.; Lewis, J.; Raff, M.; Roberts, K.; Watson, J. D.; Grimstone, A. Trends in Biochemical Sciences 1995, 20, 210-210.

[3] Howard, J.; Hyman, A. A. Nature 2003, 422, 753-758.

[4] Herrmann, H.; Bär, H.; Kreplak, L.; Strelkov, S. V.; Aebi, U. Nature Reviews Molecular Cell Biology 2007, 8, 562-573.

[5] Vavylonis, D.; Yang, Q.; O'Shaughnessy, B. Proceedings of the National Academy of Sciences of the United States of America 2005, 102, 8543-8548.

[6] Köster, S.; Weitz, D. A.; Goldman, R. D.; Aebi, U.; Herrmann, H. Current opinion in cell biology 2015, 32, 82-91.

[7] Sept, D.; Xu, J.; Pollard, T. D.; McCammon, J. A. Biophysical journal 1999, 77, 29112919.

[8] Wegner, A. Journal of molecular biology 1976, 108, 139-150.

[9] Lieleg, O.; Claessens, M. M.; Bausch, A. R. Soft Matter 2010, 6, 218-225.

[10] Conde, C.; Cáceres, A. Nature Reviews Neuroscience 2009, 10, 319-332.

[11] AMOS, L. A.; Klug, A. Journal of cell science 1974, 14, 523-549.

[12] Erickson, H. P. The Journal of cell biology 1974, 60, 153-167.

[13] Desai, A.; Mitchison, T. J. Annual review of cell and developmental biology 1997, 13, 83-117.

[14] Forscher, P.; Smith, S. J. The Journal of cell biology 1988, 107, 1505-1516.

[15] Kielbassa, K.; Schmitz, C.; Gerke, V. Experimental cell research 1998, 243, 129-141. 
[16] An, S. S.; Laudadio, R. E.; Lai, J.; Rogers, R. A.; Fredberg, J. J. American Journal of Physiology-Cell Physiology 2002, 283, C792-C801.

[17] Prabhune, M.; Belge, G.; Dotzauer, A.; Bullerdiek, J.; Radmacher, M. Micron 2012, 43, 1267-1272.

[18] Wottawah, F.; Schinkinger, S.; Lincoln, B.; Ananthakrishnan, R.; Romeyke, M.; Guck, J.; Käs, J. Physical review letters 2005, 94, 098103.

[19] Thoumine, O.; Cardoso, O.; Meister, J.-J. European Biophysics Journal 1999, 28, 222234.

[20] Yamada, K. M.; Geiger, B. Current opinion in cell biology 1997, 9, 76-85.

[21] Geiger, B.; Yehuda-Levenberg, S.; Bershadsky, A. Cells Tissues Organs 1995, 154, 46-62.

[22] Zhang, Y.; Ge, C.; Zhu, C.; Salaita, K. Nature communications 2014, 5.

[23] Grashoff, C.; Hoffman, B. D.; Brenner, M. D.; Zhou, R.; Parsons, M.; Yang, M. T.; McLean, M. A.; Sligar, S. G.; Chen, C. S.; Ha, T.; Schwartz, M. A. Nature 2010, 466, 263-266.

[24] Wang, X.; Ha, T. Science 2013, 340, 991-994.

[25] Hoffmann, B.; Schäfer, C. Cell adhesion \& migration 2010, 4, 190-193.

[26] Mattila, P. K.; Lappalainen, P. Nature reviews Molecular cell biology 2008, 9, 446-454.

[27] Small, J. V.; Herzog, M.; Anderson, K. The Journal of cell biology 1995, 129, 1275-1286.

[28] Svitkina, T. M.; Verkhovsky, A. B.; McQuade, K. M.; Borisy, G. G. The Journal of cell biology 1997, 139, 397-415.

[29] Mitchison, T.; Cramer, L. Cell 1996, 84, 371-379.

[30] Ridley, A. J.; Schwartz, M. A.; Burridge, K.; Firtel, R. A.; Ginsberg, M. H.; Borisy, G.; Parsons, J. T.; Horwitz, A. R. Science 2003, 302, 1704-1709.

[31] Yam, P. T.; Wilson, C. A.; Ji, L.; Hebert, B.; Barnhart, E. L.; Dye, N. A.; Wiseman, P. W.; Danuser, G.; Theriot, J. A. The Journal of cell biology 2007, 178, 1207-1221.

[32] Sharp, D. J.; Rogers, G. C.; Scholey, J. M. Nature 2000, 407, 41-47.

[33] Manning, A. L.; Compton, D. A. Current opinion in cell biology 2008, 20, 101-106.

[34] Nicklas, R. B. The Journal of Cell Biology 1983, 97, 542-548.

[35] Inoué, S.; Sato, H. The Journal of general physiology 1967, 50, 259-292. 
[36] Inoué, S.; Salmon, E. D. Molecular Biology of the Cell 1995, 6, 1619-1640.

[37] Westermann, S.; Drubin, D. G.; Barnes, G. Annu. Rev. Biochem. 2007, 76, 563-591.

[38] Svoboda, K.; Block, S. M. Cell 1994, 77, 773-784.

[39] Howard, J.; Hyman, A. A. Current opinion in cell biology 2007, 19, 31-35.

[40] Dumont, S.; Mitchison, T. J. Current Biology 2009, 19, R749-R761.

[41] Rodriguez, O. C.; Schaefer, A. W.; Mandato, C. A.; Forscher, P.; Bement, W. M.; Waterman-Storer, C. M. Nature cell biology 2003, 5, 599-609.

[42] Brandt, R. Cell and tissue research 1998, 292, 181-189.

[43] Scholey, J. M.; Brust-Mascher, I.; Mogilner, A. Nature 2003, 422, 746-752.

[44] Schmid-Schönbein, G.; Sung, K.; Tözeren, H.; Skalak, R.; Chien, S. Biophysical Journal 1981, 36, 243.

[45] Thoumine, O.; Ott, A. Biorheology 1997, 34, 309-326.

[46] Mizuno, D.; Bacabac, R.; Tardin, C.; Head, D.; Schmidt, C. F. Physical review letters 2009, 102, 168102.

[47] Fabry, B.; Maksym, G. N.; Butler, J. P.; Glogauer, M.; Navajas, D.; Fredberg, J. J. Physical review letters 2001, 87, 148102.

[48] Alcaraz, J.; Buscemi, L.; Grabulosa, M.; Trepat, X.; Fabry, B.; Farré, R.; Navajas, D. Biophysical journal 2003, 84, 2071-2079.

[49] Gefen, A.; Margulies, S. S. Journal of biomechanics 2004, 37, 1339-1352.

[50] Discher, D. E.; Janmey, P.; Wang, Y.-I. Science 2005, 310, 1139-1143.

[51] Solon, J.; Levental, I.; Sengupta, K.; Georges, P. C.; Janmey, P. A. Biophysical journal 2007, 93, 4453-4461.

[52] Engler, A. J.; Sen, S.; Sweeney, H. L.; Discher, D. E. Cell 2006, 126, 677-689.

[53] Gardel, M.; Nakamura, F.; Hartwig, J.; Crocker, J.; Stossel, T.; Weitz, D. Proceedings of the National Academy of Sciences of the United States of America 2006, 103, 1762-1767. 



\section{Chapter 2}

\section{DNA force sensors}

Current advances in force sensing techniques have now made it possible to measure forces in the $p N$ range in vivo. Upcoming DNA-based force sensor designs offer many advantages over traditional protein-based FRET sensors; flexibility in sequence and length giving multiple possible structures and accessibility to a broad force range, to name a few. This chapter begins with an account of progress in the field of cell mechanics and force sensing in cells over the past few years. We elaborate on the material properties of DNA that make it an optimum candidate in designing a force sensor. We then describe in detail the design of our DNA force sensor, along with control experiments that validate its working. In the later part of the chapter, we explore possibilities of force application on the sensor in different systems including isolated, immobilized DNA as well as in artificial cytoskeletal networks. 


\subsection{Advances in the field of force sensing}

The demonstration of traction forces that are exerted by cells as a function of its structure and shape modulation, congruent to the classical biochemical cues, was depicted by culturing cells on soft and deformable silicone substrates. ${ }^{112}$ The discovery that mechanical cues influence interactions of cells with their surrounding matrix introduced the field of cell mechanics. Advanced methods have since been developed for quantitatively measuring traction forces. Some of them include embedding beads in substrates, using deformable patterns or micro-posts, thus enabling force measurements in the $\mathrm{nN}$ range. 3

In the past few years, increasing efforts have been made to increase the accuracy of force measurements in cells down to the pN range. Incorporating fluorescent dyes in force sensors is a lucrative option as it combines intuitive, qualitative visualization of fluorescence with quantification of forces. Thus, a range of force sensors were introduced, principally based on distance dependent fluorescence resonance energy transfer (FRET) between two dyes.

The design of the first such molecular sensors was primarily based on protein manipulation. Typically, a flexible protein domain was inserted between a FRET pair and genetically encoded with specific proteins of interest. One of the noteworthy examples of protein-based force sensors was insertion of a tension sensor module in vinculin to quantitatively investigate its role in focal adhesions. ${ }^{7}$ The authors could quantify forces across vinculin with $\mathrm{pN}$ resolution, contributing largely to our understanding of focal adhesion dynamics. Along similar lines, another set of experiments fused a casette of FRET pair, separated by an alpha helix, with $\alpha$-actinin and expressed it in human cells. The work showed stress changes in $\alpha$-actinin under various conditions such as cell migration, increased cell contractility with thrombin and osmotic changes. $\frac{[89]}{}$ Another application of a protein-based fluorescence sensor includes visualization of the force distribution on integrins with single molecule sensitivity. 10

On parallel lines of research, other works explored new designs of force sensors, mostly based on DNA. The 'tension gauge tether' (TGT) was an important development that exploited the dependence of the rupture force of double-stranded DNA on the position of force application. 11 By utilizing different force geometries from unzipping to shearing of DNA strands, a force range from 12-56 pN was available for measuring the forces exerted on integrin-ligand receptors. Building on this foundation, more DNA-based sensor designs were introduced with additional features such as high spatio-temporal resolution. DNA hairpins incorporating a fluorophorequencher pair were used as elegant sensors for measuring single integrin forces. $12[13$

The latest developments in force sensors have now made it possible to measure forces at the scale of single molecules. However, measuring tension across integrins to reveal the intricate details of cell adhesion to substrates remains prime focus of most experiments. This limits force sensing to a 2D level while ignoring the intra and inter-filament interactions between cytoskeletal filaments, which are the main force generating machinery inside cells. $\left.{ }^{14}-16\right]$ Also, the forces involved in cellular processes such as adhesion, migration or division have been studied individually. ${ }_{[5]+17-19}$ What is missing, however, is a general force sensor as a tool to investigate multiple processes, giving a broad overview of cellular functioning.

Our goal was to bridge this gap by designing a DNA-based force sensor for 3D force sensing 
in cells. The sensor can be attached to cytoskeletal filaments, mainly actin and microtubules, to measure interactive forces between biopolymers. Finally, the spatial information of force distributions can be utilized to realize a coarse-grained stress map of the cell.

\subsection{DNA structure}

Prior to elaborating on the concept of a DNA-based force sensor, it is first important to understand the structural properties of DNA that make it such an interesting material in general and, as a component of force sensor, in particular.

DNA stands for deoxyribonucleic acid. It is a biopolymer responsible for encoding genetic information in cells. Fig. 2.1 shows the structure of DNA, consisting of two complementary strands wound around each other to form a double helix.20 Each strand is composed of a deoxyribose sugar phosphate backbone and 4 nucleotide bases - adenine $(A)$, thymine $(T)$, cytosine $(C)$ and guanine $(G)$. The nucleotide bases pair with each other with high specificity; adenine with thymine (A-T) and cytosine with guanine $(\mathrm{G}-\mathrm{C})$. A complementary DNA sequence refers to matching sequence of bases on two strands such that they can pair together. The length between two base pairs is known to be around $0.33 \mathrm{~nm}$. Therefore, DNA of desired length can be generated with high precision by controlling the number of base pairs.

The mechanical properties of DNA have been extensively studied and are well characterized. DNA is a flexible polymer with a persistence length of approximately $50 \mathrm{~nm}$ and best described by the worm like chain (WLC) model.2122 The

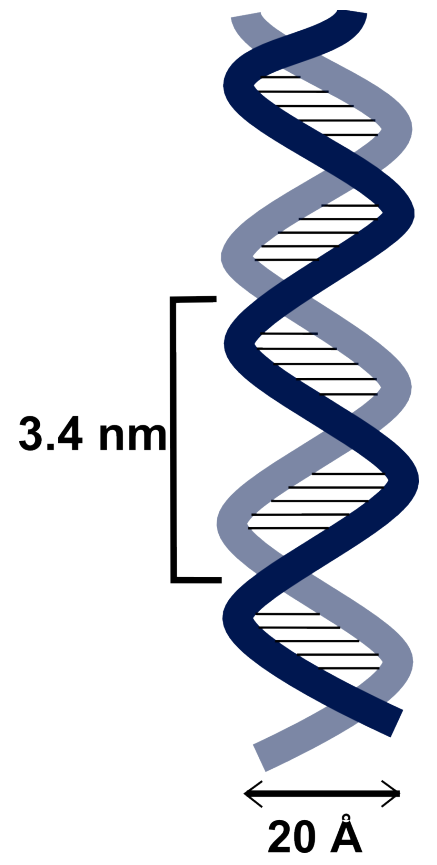

Figure 2.1: Structure of doublestranded DNA. strand separation forces of double-stranded DNA have also been quantified and are known to depend on a number of factors; an example being GC content of the sequence. The hydrogen bonds between the base pairs maintain the double-stranded structure of DNA. As guanine interacts with cytosine through three hydrogen bonds while adenine and thymine have only two, DNA with high GC content requires larger mechanical forces for strand separation. 23

Thus, we have a monodisperse material with precise length control and well characterized mechanical properties that can be easily manipulated to form different structures, based on its specificity to complementary sequences. These properties make DNA a perfect building block for novel structures and designs. The field of DNA origami, as it is popularly called, has found wide applications in the past decade. ${ }^{[24-26}$ The following section gives the advantages of choosing DNA as a candidate for designing a force sensor over traditional protein-based sensors. 


\subsection{Advantages of a DNA-based force sensor}

There are certain important criteria that need to be considered in designing a molecular force sensor. First and foremost, it should easily self-assemble with high efficiency and minimum errors. DNA strands have been shown to self-assemble into 3D structures under hybridization conducive environment. The assembly process is in order of few seconds and the yield can be as good as upto $95 \% .27$ The flexibility of having a vast array of possible designs based on DNA sequences gives DNA-based designs an advantage over protein-based constructs.

(A) Unzipping

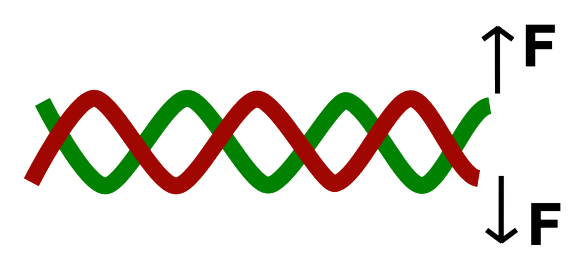

(B)

\section{Shearing}

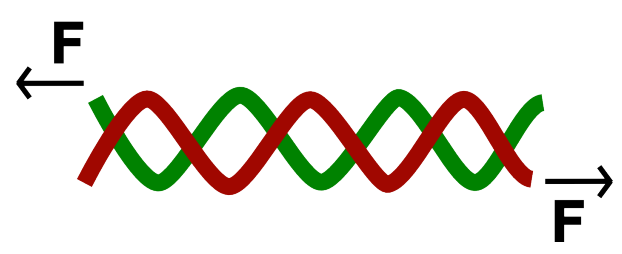

Figure 2.2: Mechanisms of separation of complementary DNA strands. (A) Applying forces on same end of DNA in opposite direction results in unzipping of DNA. (B) Pulling on opposite ends of DNA requires relatively high rupture forces than unzipping of DNA.

Another desirable property of a force sensor is that it should be possible to access a broad range of forces, without modifying the design in a major way. Protein-based sensors, for instance, are limited to a force range of approximately 2-6 pN, rendering them useless in certain biological processes where forces exceed $50 \mathrm{pN} . \stackrel{1113}{113}$ On the contrary, DNA sensors can be easily manipulated to generate various sensor designs that respond to different forces. This can be achieved through several mechanisms. It is known that the unbinding forces required for separation of double-stranded DNA vary depending on the length, temperature or GC content of the sequence. ${ }^{2328+31}$ Also, strand separation forces vary largely depending on the mechanism of pulling. ${ }^{23 / 32}$ A simple way of understanding this concept is to imagine a DNA duplex of complementary strands with forces exerted at its ends. Applying forces in opposite directions from same end of the duplex results in unzipping of DNA and requires relatively small force for strand separation, as shown in Fig. 2.2 A. On the other hand, shearing the DNA by pulling the strands from opposite ends will require a larger force, as shown in Fig. 2.2B. Therefore, the same design of DNA sensor can be fine tuned to measure over a broad force range by simply modifying the nature of sequence or the force application geometry. A recent study showed rupture forces for a 21 bp DNA strand to span a range between $12-56 \mathrm{pN}$, based on the force application geometry. 11

Lastly, the possibility of introducing the force sensor into cells while it maintains its structure and function is a vital prerequisite in our experiments. Although the introduction of foreign DNA might trigger cellular defense mechanisms, experiments have shown DNA tetrahedra complex to remain intact in mammalian cells for $48 \mathrm{hrs}$ without degradation. $27 / 33$ Therefore, DNA force sensors can be expected to survive in cells over the duration of measurements. 
In the above section we have discussed the advantages of DNA as a force sensor. As we inch a step forward in designing a DNA-based force sensor, we exploit FRET mechanisms to have a reliable visual readout from our sensor. The underlying principles of FRET are described in the following section.

\subsection{Fluorescence resonance energy transfer (FRET)}

(A)

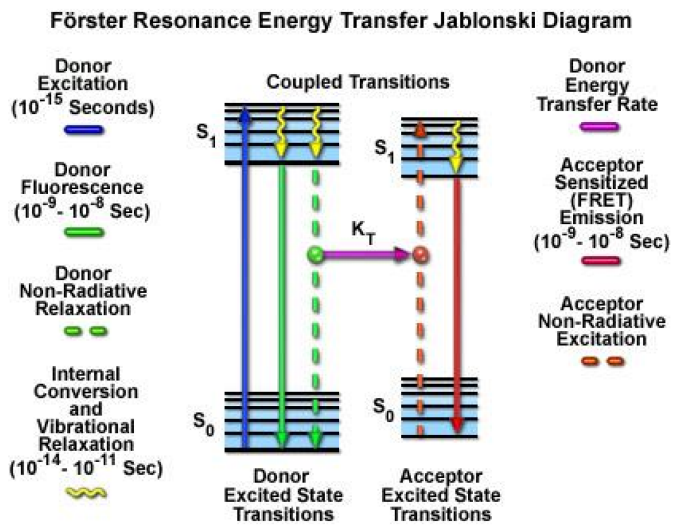

(B)

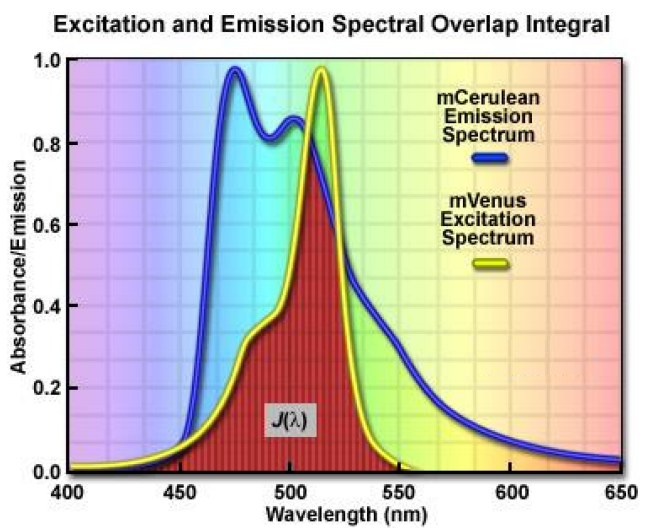

Figure 2.3: Mechanism of FRET. (A) Jablonski diagram showing energy state transitions in FRET. Blue, green and red vertical arrows represent the absorption and emission transitions and wavy yellow arrows show vibrational relaxation. The dashed lines show the energy transitions if the process would be photon-mediated, while the violet arrow indicates transfer of excited state energy from donor directly to the acceptor without emitting a photon. (B) Example of a FRET pair showing spectral overlap, where $J(\lambda)$ is the overlap integral. Figures adapted from www.microscopyu.com webpage.

Fluorescence (or Förster) resonance energy transfer, originally developed by Theodor Förster, is a mechanism that occurs between two fluorophores in close proximity. $\frac{34}{1 n}$ FRET, one fluorophore (donor) transfers its energy, on excitation, to a neighboring fluorophore (acceptor) in a non-radiative fashion. Non-radiative means that there is no emission of photons from the excited fluorophore. Fig. 2.3A shows a Jablonski energy diagram that illustrates energy transitions between the donor emission and acceptor absorbance in FRET. The acceptor fluorophore, excited via energy transfer from donor, undergoes emission resulting in a fluorescence signal. The distance dependent dynamics are monitored as intensity changes in donor as well as acceptor channels. However, it is not necessary to have a fluorophore as an acceptor. Dark quenchers, which return to ground state through non-radiative decay, can also be used as per experimental requirements. ${ }^{[35}$ In this case, we detect the fluorescence fluctuations of the donor itself.

There are two basic conditions required for FRET in dye molecules. First, that the absorption spectrum of the acceptor must overlap with the emission spectrum of the donor. A measure of spectral overlap is given by the overlap integral $J(\lambda)$, as shown in Fig $2.3 \mathrm{~B}$. Secondly, the separation distance between the dyes should be between $1-10 \mathrm{~nm}$. This is important as the 
energy transfer is highly distance dependent and varies as the inverse sixth power of the distance between the two dyes. The equation is given by,

$$
E_{F R E T}=\frac{1}{\left[1+\left(r / R_{0}\right)^{6}\right]},
$$

where $E$ is the FRET efficiency, $r$ is distance between the two molecules and $R_{0}$ is the characteristic distance when the FRET efficiency is 50 percent.

An important advantage of FRET is that it gives spatial information regarding two molecules with a much higher resolution than conventional fluorescence microscopy techniques. It has therefore been applied in diverse studies such as protein unfolding, protein-protein interactions and co-localization. ${ }^{36 / 37}$ As described in Section. 2.1. FRET has gained popularity in force sensor designs due to the simple fluorescent readout of a structural change, following an application of force.

Having laid the foundation of the desirable parameters for an optimum sensor design, we describe the actual design and working principle of our construct in the following section.

\subsection{Design of the DNA force sensor}

We chose a simple DNA hairpin structure as the basis of our force sensor. It consists of a 72 bases long DNA strand that folds over itself to form a hairpin of $8 \mathrm{bp}$ stem and $16 \mathrm{bp}$ loop region. Complementary strands to arms of the hairpin are 20 bases each in length. Sequences of DNA strands were designed online on NUPACK webpage and obtained commercially (Integrated DNA Technologies, Leuven, Belgium) ${ }^{38}$ The unfolding forces of DNA hairpins for a given set of parameters such as length, temperature, GC content are well characterized. 39 -41 Therefore, we can estimate forces for unfolding our DNA hairpin sensor to be approximately $10 \mathrm{pN}$.13/41

The next step was to incorporate a FRET pair in the sensor, such that unfolding of hairpin results in a visually detectable change in fluorescence. In our sensor, we use a FRET pair consisting of a fluorophore, Alexa 488 and a quencher, lowa black dark quencher. A dark quencher, as described in Section. 2.4, allows energy transfer from the fluorophore but does not emit any fluorescence. Therefore, a quenched fluorophore results in a 'dark' or low fluorescence state of the sensor.

The two main sensor designs can be categorized as reversible or irreversible. Each of these designs is described in detail below. For the sake of convenience, the strand with fluorophore and the quencher will be referred to as $F$ and $Q$, respectively. The hairpin strand will be denoted as $\mathrm{H}$, the control strand as $\mathrm{C}$. The mechanism of control strand differs with the type of sensor and will be explained while describing each of the relevant sensor.

\subsubsection{Irreversible sensor}

The design of the irreversible sensor is shown in Fig.2.4A. It consists of a self-assembled 72 bases hairpin, as described above. An additional pair of DNA strands, F and $Q$, also form a 


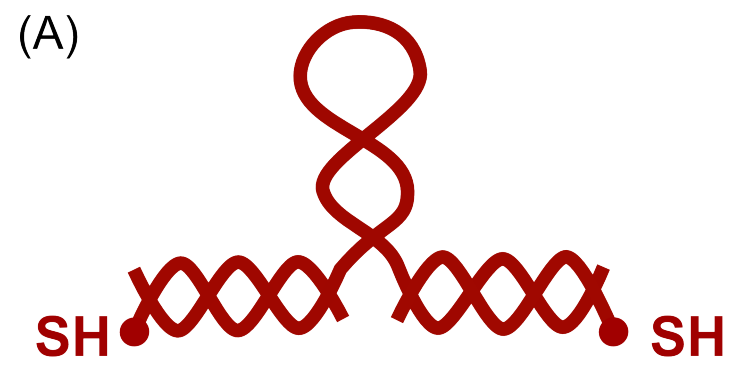

${ }_{\mathrm{F}}^{\mathrm{D}} \mathrm{DOON \textrm {N }}$
(B)

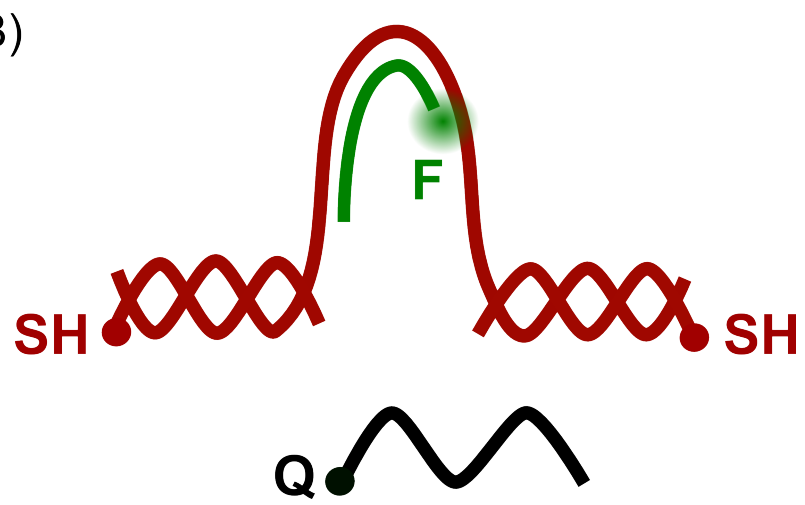

Figure 2.4: Schematic of irreversible DNA sensor. The basic design is a DNA hairpin and an additional pair of strands incorporating a fluorophore-quencher pair. Ends of sensor are modified with thiols for attachment to substrates or cytoskeletal filaments. (A) Closed configuration of DNA hairpin keeps the fluorophore (F) and quencher (Q) strands together. (B) Opening of hairpin on application of force (approximately $10 \mathrm{pN}$ ) reveals a complementary sequence for $F$ strand. This causes displacement of $F$ strand from $Q$ strand and thereby gives an increase in fluorescence. The sensor is irreversible as the hairpin remains open even after stress relaxation.

part of the sensor. F strand has 16 bases and consists of a fluorophore, Alexa 488, at one end. $Q$ strand is complementary to $F$, but is shorter (8 bases) and has a dark quencher, lowa black, at its end. The sequences of strands $F$ and $Q$ are such that $F$ is also complementary to the hairpin stem and loop. As long as the hairpin is closed, F strand remains hybridized with $Q$ strand. However, when the hairpin unfolds due to an external force, it will expose the complementary sequence to $F$ strand. This results in an energetically favorable strand displacement reaction, in which $Q$ strand is displaced, as shown in Fig. 2.5B. This results in an increase in the fluorescence intensity. Thus, we have a bright on-state of unfolded hairpin and dark off-state of folded hairpin.

The ends of the complementary strands to the arms of the hairpin are modified with a thiol $(\mathrm{SH})$ group. This is useful for attachment of the sensor to cytoskeletal filaments or substrates, as described in later sections.

\section{Control experiments}

Fig. 2.5 shows the hybridization and displacement of $F$ and $Q$ strands in bulk experiments in a spectrophotometer. DNA strands, F, Q and C, were added sequentially to hybridization buffer in a cuvette $(100 \mu \mathrm{l})$ such that the final concentration of each strand was $25 \mathrm{nM}$. Samples were excited at a wavelength of $494 \mathrm{~nm}$ and maximum emission was recorded at $520 \mathrm{~nm}$. Fluorescence intensity of F strands showed approximately 3-fold decrease on hybridization with $\mathrm{Q}$ strands. A control strand (C) was designed such that it has the same sequence as the stem and loop of the hairpin. Therefore, the control strand is perfectly complementary to the $F$ strand. Addition of the $C$ strands resulted in an energetically favorable displacement of $F$ 

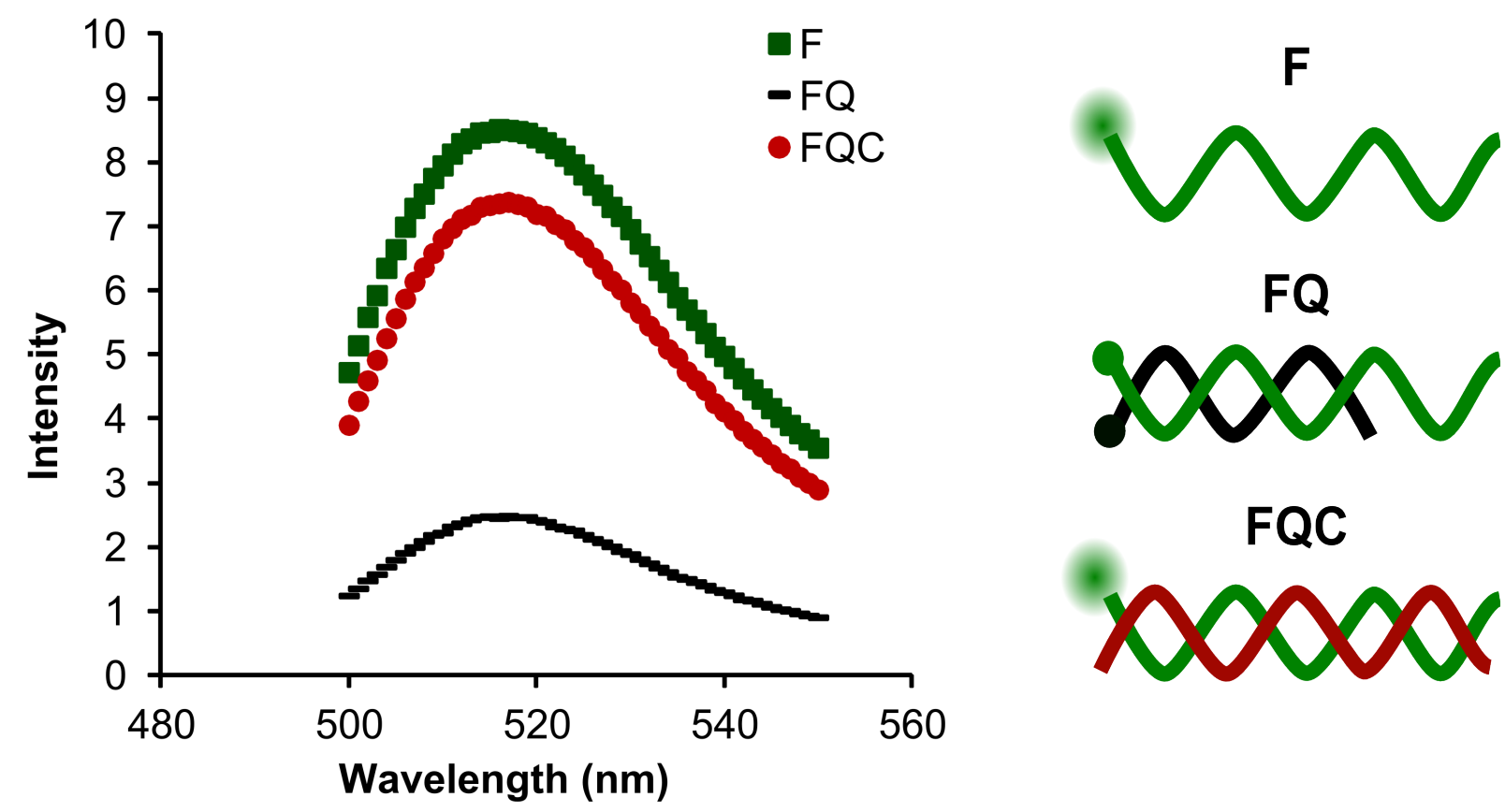

\section{FQC}

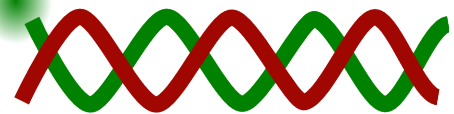

Figure 2.5: Control experiments on irreversible sensor. DNA strands, $F, Q$ and $C$ were sequentially added in a hybridization buffer such that the final concentration of each strand was $25 \mathrm{nM}$. The fluorescence was measured with a spectophotometer at a maximum emission wavelength of $520 \mathrm{~nm}$ and excitation wavelength of $494 \mathrm{~nm}$. Fluorophore (F) strands were added to measure maximum intensity, followed by the addition of quencher (Q) strands. Quenching results in a drop in the fluorescence intensity, which recovers on addition of control strands (C). Sequence of the control strand resembles part of the hairpin sequence that is complementary to the $F$ strand, thus displacing it. Schematic of $F, F Q$ and $F Q C$ strands is shown on the right.

strands from $Q$ strands, resulting in an increase in the fluorescence intensity. The fluorescence does not completely recover to its original value within the time duration of the experiment, as all the reactions are diffusion limited.

The displaced $F$ strand remains hybridized with a part of the hairpin. Thus, the sensor cannot refold on stress relaxation, making it irreversible. An advantage of irreversible sensor is that we can detect the signal for fast dynamic processes even after relaxation.

\subsubsection{Reversible sensor}

Although an irreversible sensor has certain advantages, an ideal sensor must be applicable to studying dynamic processes. Therefore, we designed an alternative reversible force sensor. Fig. 2.6 shows the basic design, which consists of a hairpin of 72 bases with thiol modified ends on complementary strands of its arms. In this sensor design, the fluorophore and quencher are attached to interior ends of arms of the hairpin. Unfolding of hairpin on force application will 

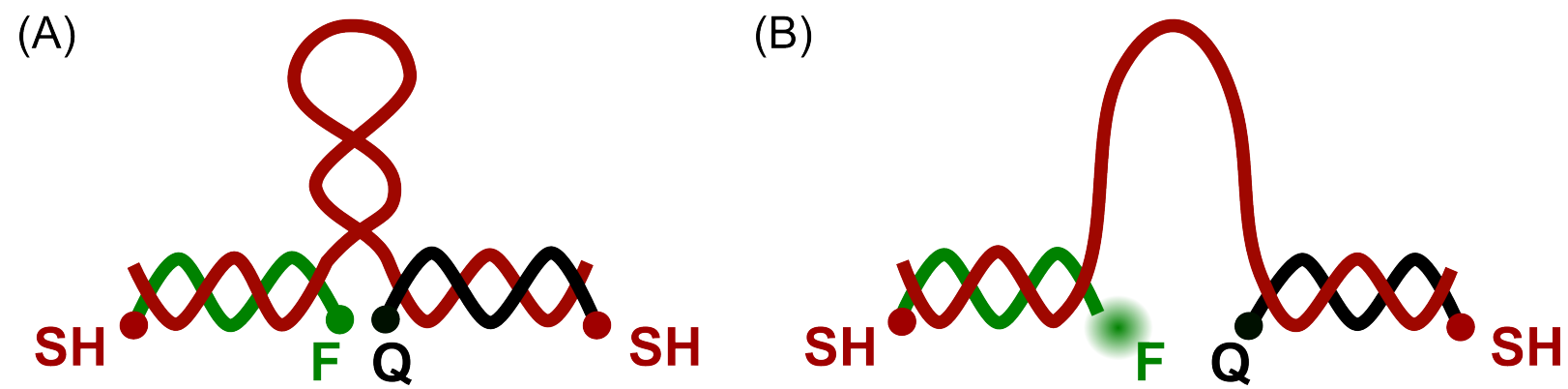

Figure 2.6: Schematic of reversible DNA sensor. The basic design is a DNA hairpin incorporating a fluorophore-quencher pair. Ends of the sensor are modified with thiols for attachment to substrates or cytoskeletal filaments. (A) Closed configuration of DNA hairpin will have low fluorescence due to FRET between fluorophore and quencher. (B) Applying forces (approximately $10 \mathrm{pN}$ ) at ends of the sensor results in unfolding of the hairpin, which increases distance between the FRET pair. An open configuration of DNA hairpin, thus, corresponds to higher fluorescence than closed configuration. The sensor is reversible as the hairpin closes after stress relaxation and can undergo multiple on-off cycles.
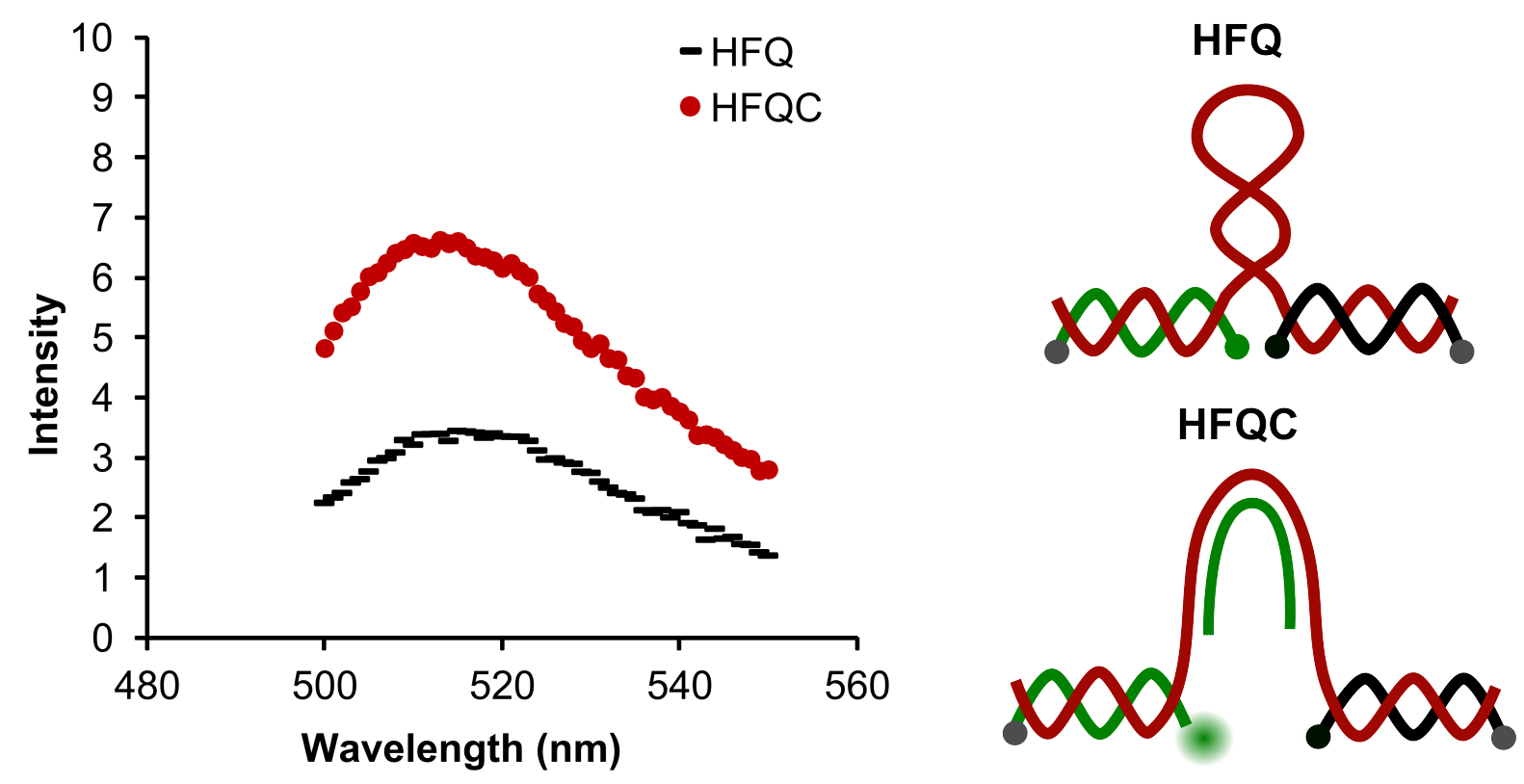

Figure 2.7: Control experiments on a reversible DNA sensor. DNA sensor was assembled by mixing $H, F$ and $Q$ strands in a hybridization buffer such that the final concentration of each strand was $25 \mathrm{nM}$. The fluorescence was measured with a spectrophotometer at a maximum emission wavelength of $520 \mathrm{~nm}$ and excitation wavelength of $494 \mathrm{~nm}$. Closed state of DNA sensor corresponds to a basal fluorescence intensity. Addition of control (C) strands, which are complementary to stem as well as loop of hairpin, unfold the hairpin resulting in an increase in fluorescence. A schematic of HFQ and HFQC strands is shown on the right. 
result in an increase in the fluorescence intensity, as shown in Fig. 2.6B. An important feature of this sensor is that the hairpin can refold on relaxation of an applied force. Thus, the sensor can reversibly switch between folded and unfolded states, allowing us to monitor multiple on-off cycles until the fluorophore bleaches.

\section{Bulk control experiments}

Fig. 2.7 shows control experiments of the reversible sensor measured with a spectrophotometer, similar to those of the irreversible sensor. DNA sensor, $H F Q$, was assembled by mixing $H, F$ and $Q$ strands in a hybridization buffer such that the final concentration of each strand was $25 \mathrm{nM}$. Emission scans of the sensor in its folded configuration were measured at an excitation wavelength of $494 \mathrm{~nm}$. Although the hairpin is in its 'off-state', there is basal level fluorescence depending on efficiency of quencher and due to the fraction of unhybridized $F$ strands. A control (C) strand, which is complementary to stem and part of the hairpin loop, was then added to HFQ. Competitive binding of the control strand to the sensor forms the HFQC complex, resulting in unfolding of the hairpin. The resultant increase in fluorescence successfully demonstrates the working principle of the reversible sensor in a bulk solution.

\section{Single molecule control experiments}

Control experiments demonstrating working of the construct were also performed at a single molecule level. $1 \mathrm{nM}$ of HF and HFQ were spin coated on a plasma cleaned coverslip at 8000 rpm for $40 \mathrm{~s}$. The scan pixel size was $100 \mathrm{~nm}$ with a pixel dwell time of $5 \mathrm{~ms}$. Fig. 2.8 shows the intensity scans from a commercial confocal system Microtime 200 (PicoQuant, Berlin, Germany). Scans of immobilized HF show clearly visible single molecules in Fig. 2.8A. The quenched HFQ scans, however, have much a weaker intensity as seen in Fig. 2.8B. We identified single molecules from the images using a 2D Gaussian model and then plotted the histograms of the maximum intensity pixel for each molecule. Fig. 2.8C, D show histograms of 288 and 71 identified HF and HFQ molecules, respectively. The average of the distributions are 211 and 65 photons per pixel, respectively.

\subsection{Immobilized force sensor experiments}

The next step was to demonstrate unfolding of hairpins in response to externally applied forces. We began with a simple system of immobilizing DNA on a surface, which would then be subjected to forces. The advantage of this system is its minimum complexity, as we work with a single component. These experiments would also be useful in verification of the force range required for unfolding hairpins.

Plasma cleaned coverslips were silanized and coated with sulfo-SMCC to bind thiol modified DNA following a protocol described in detail in Chapter 6. A schematic of the coating protocol 


\section{(A)}

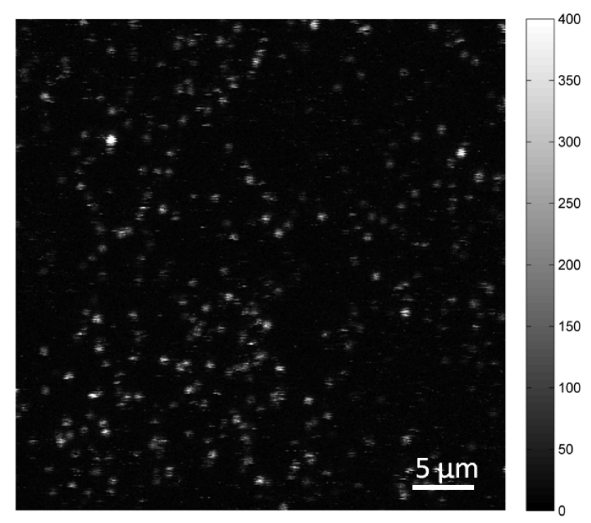

(C)

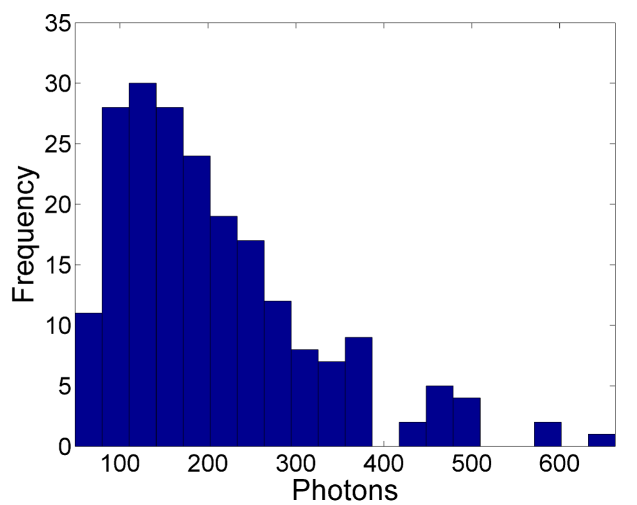

(B)

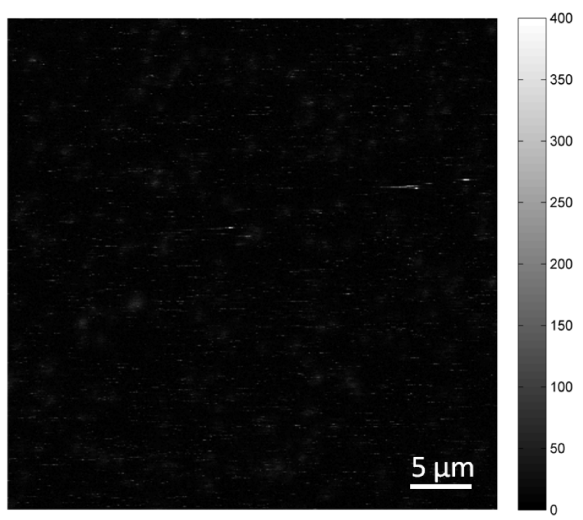

(D)

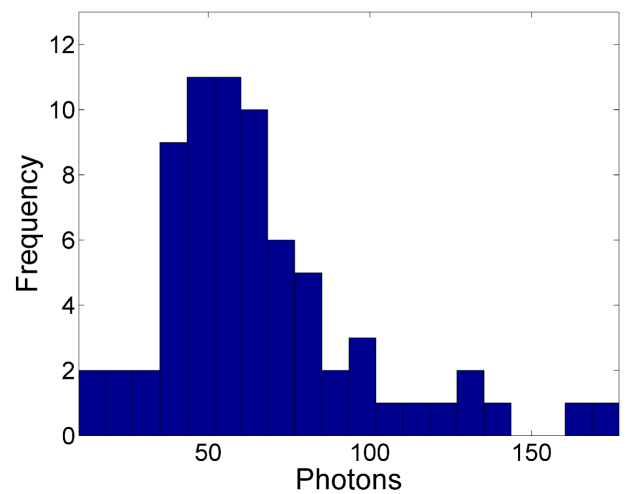

Figure 2.8: Single molecule controls of DNA sensor. Confocal scans of immobilized (A) HF strands and (B) HFQ strands show clear differences in the fluorescence intensity after quenching. (C) and (D) represent histograms of maximum photon counts per pixel from the identified 2D Gaussian single molecule patterns from (A) and (B), respectively.

is shown in Fig. 2.9A. The binding specificity of the coated coverslip was tested by spotting different DNA strands such as HF, HFQ, HFQC and washing thoroughly. F strand with biotin modification, instead of thiol, was also spotted as a negative control. Fluorescence microscopy (data not shown) revealed that the biotinylated strand was almost completely washed off, while the thiolated DNA remained attached to the surface. This verified the specificity of the coated coverslip for thiol modified DNA. For certain experiments the coverslip was then attached to a sticky-Slide I Luer (Ibidi GmbH, Martinsried, Germany) with a channel height of approximately $100 \mu \mathrm{m}$.

We confirmed immobilization and in situ hybridization of DNA strands with the following experiment. HF strands were spotted on coated coverslip and images were recorded in an epifluorescence microscope. Q strands were flushed into the channel and washed after $1 \mathrm{hr}$ of incubation. Images of the same spot were recorded and the procedure was repeated after flushing in control strands. Fluorescence intensities from images of HF, HFQ and HFQC 
(A)

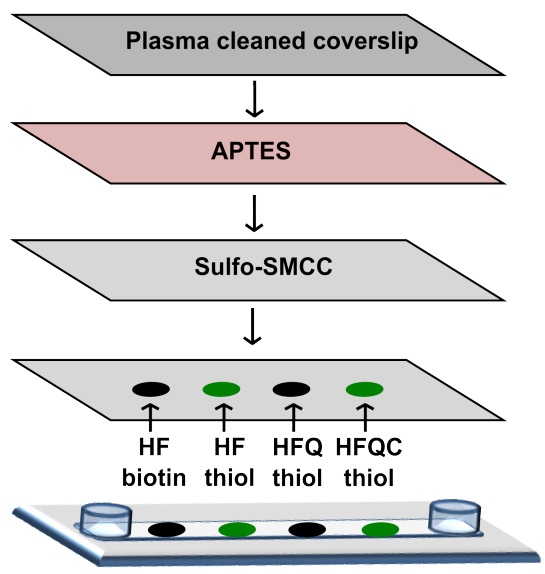

(B)

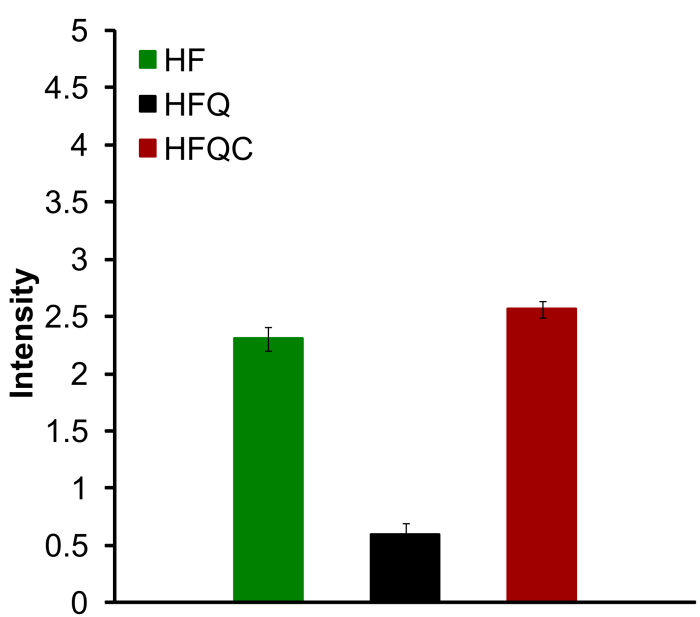

Figure 2.9: Immobilization of thiolated DNA on coated coverslips. (A) Plasma coated coverslips were silanized with APTES, followed by sulfo-SMCC treatment. Biotinylated HF, thiol ended HF, HFQ and HFQC strands were spotted on coverslip and enclosed in a $100 \mu \mathrm{m}$ channel. (B) Hybridization of immobilized DNA is demonstrated by spotting thiolated $\mathrm{HF}$ strand and sequentially adding $\mathrm{Q}$ and $\mathrm{C}$ strands. Images were recorded after $1 \mathrm{hr}$ on addition of each type of strands. Intensities show quenching and recovery of fluorescence after flushing in $Q$ and $C$ strands, respectively. Error bars show standard error of mean.

strands are shown in Fig. 2.9B. Quenched HFQ strands showed reduced fluorescence intensity in comparison to HF strands. The addition of control strands (HFQC) resulted in an increase in the fluorescence intensity. This was consistent with our observations from bulk experiments in spectrophotometer. Thus, we could confirm that DNA was successfully immobilized and capable of hybridization with complementary strands in its immobilized state.

The next step was to unfold the immobilized hairpins using external force and record the corresponding changes in fluorescence. We tested various methods of force applications in parallel for the same; elaborated below. The reversible DNA sensor was used in all the following experiments, unless mentioned otherwise.

\subsubsection{Magnetic beads experiments}

A quick and easy method of exerting forces was to use a magnet to pull on magnetic beads attached to the DNA sensor. Streptavidin coated magnetic beads were obtained commercially as Dynabeads M-280 Streptavidin (Life Technologies GmbH, Darmstadt, Germany). Fig. 2.10A shows a schematic of the experimental set-up. HF strands were immobilized on coated coverslips and hybridized with biotinylated $Q$ strands. The streptavidin coated magnetic beads were then attached to the immobilized DNA sensors. A small magnet was brought in vicinity of the coverslip, while simultaneously recording images. The presence of magnet resulted in visible movement of beads, except the few which remained strongly adhered to the substrate. However, the beads showed a strong autofluorescence at an excitation wavelength of $494 \mathrm{~nm}$, which 
(A)

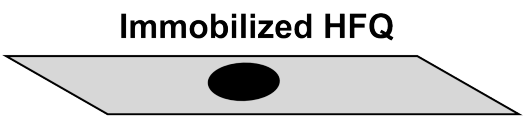

Add neutravidin coated magnetic beads

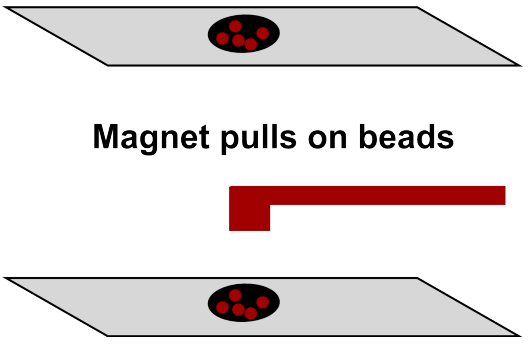

(B)

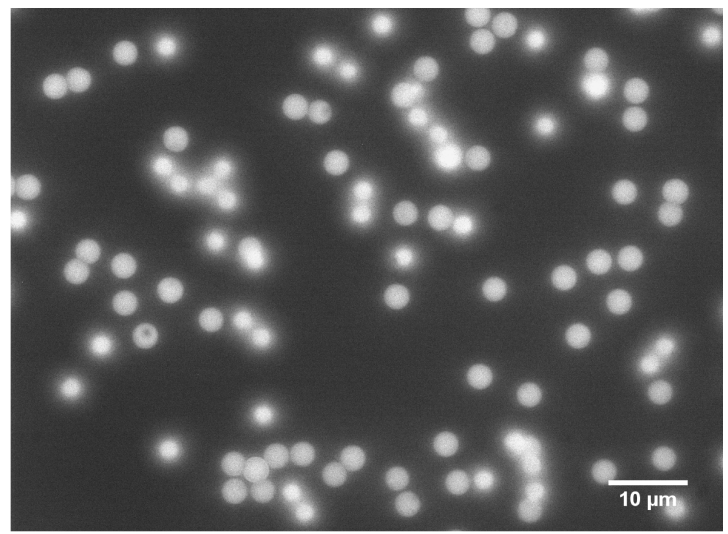

Figure 2.10: Magnetic beads to pull on immobilized DNA sensors. (A) Streptavidin coated magnetic beads were incubated with immobilized HFQ strands ( $Q$ strands with modified biotin ends). Beads were subjected to magnetic forces using a small board magnet. (B) Magnetic beads were autofluorescent at the excitation wavelength of the sensor, which probably masked any changes in the fluorescence intensity given by opening of the hairpins.

(A)

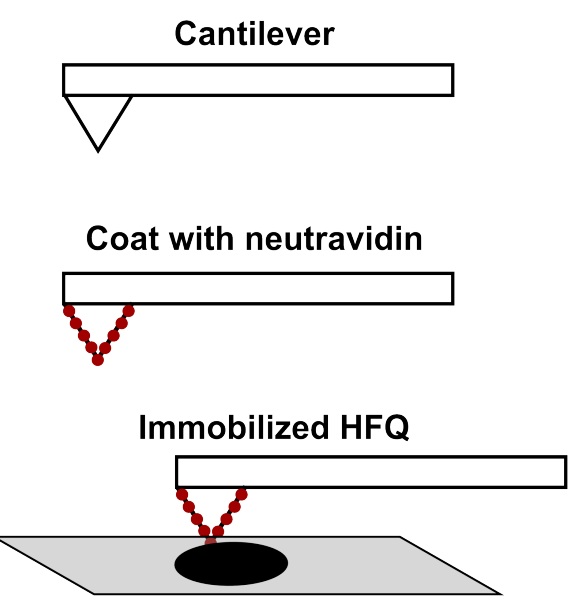

(B)

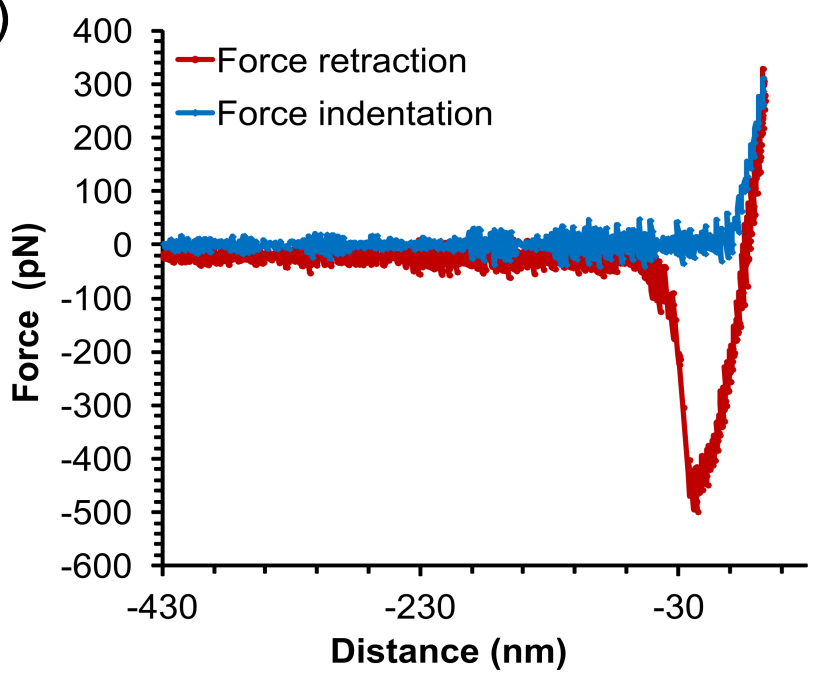

Figure 2.11: AFM experiments on immobilized DNA sensors. (A) An AFM cantilever tip was coated with neutravidin and interacted with HFQ (biotinylated $Q$ ) strands that were immobilized on a coverslip. (B) A representative force curve was measured in contact mode with an indentation force of $300 \mathrm{pN}$, waiting time of $1 \mathrm{~s}$ and retraction distance of $500 \mathrm{~nm}$. The tip reacts non-specifically with the surface resulting in large forces during retraction; possibly ripping the immobilized DNA strands off the surface. 
overlaps with the spectrum of fluorescent DNA, as shown in Fig. $2.10 \mathrm{~B}$. Thus, any fluorescence from opening of hairpins due to movement of beads would be masked and difficult to detect in presence of the autofluorescent beads.

\subsubsection{AFM experiments}

Another strategy was to apply forces on immobilized DNA sensor using atomic force microscopy (AFM). AFM has been commonly used to determine ligand-protein interaction forces by using functionalized cantilever tips. ${ }^{42-45}$ In our experiments, we used neutravidin functionalized cantilever tip to pull on biotinylated DNA strands, as described below.

HFQ complex was hybridized in a final concentration of $1 \mathrm{nM}$ using $\mathrm{F}$ strand with thiol modification at end, hairpin and quencher strand with biotinylated end. The strands were immobilized on a sulfo-SMCC coated coverslip. The cantilever (Olympus Biolever RC150VB) was coated with neutravidin using a protocol described in Chapter 6. Schematic of experimental set-up is shown in Fig. 2.11A.

Experiments were performed on a MFP3D AFM set-up (Asylum Research, Santa Barbara, CA) combined with a TIRF microscope. The details of the experimental set-up are described elsewhere. ${ }^{46}$ The TIRF microscope was equipped with a CFI Apochromat TIRF 100x lens (Nikon $\mathrm{GmbH}$, Düsseldorf) and an Andor DL-658M-OEM (Andor Technology, Belfast, UK) camera. Samples were excited with a 488 laser and images were recorded with an exposure time of $0.1 \mathrm{~s}$. We operated AFM in contact mode with an approach force of $300 \mathrm{pN}$, waited for $1 \mathrm{~s}$ and retracted the cantilever for $500 \mathrm{~nm}$ at a velocity of $1 \mu \mathrm{m} / \mathrm{s}$. A representative force curve obtained at these settings is shown in Fig. $2.11 \mathrm{~B}$. As seen from the force retraction curve, the cantilever tip adhered to the surface with non-specific interactions. Detachment of tip from the surface required a large force (approximately $500 \mathrm{pN}$ ) that probably also resulted in ripping off the immobilized DNA strands from the surface.

We also experienced problems in optimizing concentration of DNA strands for our experiments. As seen from bulk experiments, quenched DNA shows some basal level fluorescence. High concentration of immobilized DNA resulted in a large background signal in the TIRF microscope, making it difficult to detect any changes in fluorescence. Low concentration of DNA, on the other hand, resulted in reduced number of hairpins that could interact with the AFM tip. 


\subsubsection{Flow experiments}

Lastly, we used shear flow in a microfluidic chamber to apply forces on DNA hairpins. HFQ strands; thiol modified $F$ strands and $Q$ strands with modified biotin ends were spotted on a coated coverslip. Silica beads of $5 \mu \mathrm{m}$ diameter were coated with neutravidin and then incubated with immobilized HFQ strands to allow interaction of streptavidin with biotinylated ends of $Q$ strands. Inclusion of beads was necessary to ensure sufficient shear forces on the construct. This is because DNA is small in size, with a maximum contour length of approximately $25 \mathrm{~nm}$. As the flow velocity close to the surface is negligible, the hydrodynamic forces at surface of the chamber would be insufficient to open the hairpins. DNA strands, along with attached beads, were enclosed in a $100 \mu \mathrm{m}$ channel with sticky-Slide I Luer (Ibidi GmbH, Martinsried, Germany). A syringe pump (Harvard Apparatus, Holliston, MA, USA) was used to maintain a fixed flow rate. We tested a wide

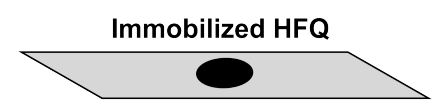

Add neutravidin coated silica beads

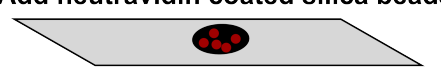

Shear flow through chamber

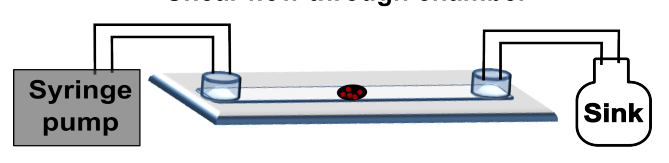

Figure 2.12: Shear flow to unfold DNA hairpins. Silica beads of $5 \mu \mathrm{m}$ diameter were coated with neutravidin and attached to biotin ends of $Q$ strand on an immobilized HFQ complex. The DNA strands with attached beads were enclosed in a $100 \mu \mathrm{m}$ high channel. A syringe pump was used to flow buffer into the channel at a constant flow rate. range of flow rates and yet did not observe an increase in fluorescence intensity of the DNA sensors.

In this chapter, we discussed the need for novel 3D force sensor designs to explore force sensing and stress mapping in cells. DNA-based force sensor designs provide various advantages over protein-based ones; most importantly, flexibility in designs and possibility to detect a broad force range. We have, to this end, designed a simple DNA-based hairpin sensor incorporating a FRET pair to give a visual output on application of force. We could also successfully demonstrate working of our sensor with control experiments in bulk, as well at single molecule level.

The force application experiments on immobilized DNA turned out to be more challenging than expected. AFM and magnetic bead experiments posed fundamental problems, making these methods unsuitable for our experiments. In shear flow experiments, estimated forces for unfolding the DNA hairpin are based on the assumption that the sensor is oriented perpendicular to the surface. Unfortunately, we have no information regarding orientation of the sensor. We speculate that horizontally oriented sensor that remains stuck in the plane of the coverslip might potentially hinder opening of hairpin on application of shear force.

Successfully unfolding immobilized DNA hairpins would have demonstrated working of the DNA sensor in a simple system. However, unprecedented situations added to complications, 
demanding time consuming controls before further execution of these experiments. As we aim to measure forces in a 3D environment in cells, we redirected our focus to design an experimental system that better resembles cellular interior. In vitro composite networks of crosslinked cytoskeletal filaments have been shown to resemble living cells in their mechanical properties. ${ }^{47}$ Attaching DNA force sensors to in vitro cytoskeletal filament networks, which can be perturbed by external forces, would serve as an ideal system for force sensing experiments. Moreover, crosslinking cytoskeletal filaments with DNA gives a model network with tunable crosslinker parameters.

Therefore, we generated in vitro networks of microtubules crosslinked with DNA sensor. This system serves dual goals of providing an ideal experimental system to test force sensing as well as for studying mechanics of composite networks. The crosslinking and characterization of mechanics of these networks is described in detail in Chapter 3. The next section describes experiments monitoring response of DNA sensor in microtubule networks, after application of external shear forces.

\subsection{Force sensing in cytoskeletal networks}

Microtubules were crosslinked with the DNA sensor via a heterobifunctional chemical crosslinker, sulfo-SMCC. The networks were first subjected to bulk shear, followed by experiments where microscopic shear was applied by local perturbation of network.

\subsubsection{Shearing with needle in a cuvette}

We first tested working of the irreversible sensor in in vitro networks through a simple, crude experiment. Microtubules were crosslinked with DNA sensor in a cuvette and vigorously sheared using a microliter needle (Hamilton Process Analytics, Switzerland) with an inner diameter of $150 \mu \mathrm{m}$. A schematic is shown in Fig. $2.13 \mathrm{~A}$. The fluorescence intensities of pre-sheared and post-sheared networks were measured in a spectrophotometer (maximum excitation 494 $\mathrm{nm}$, maximum emission $520 \mathrm{~nm}$ ). The graph shown in Fig. 2.13 $\mathrm{B}$ shows an increase in the fluorescence intensity on shearing the networks; corresponding to displacement of F strand from $Q$ strand on opening of the hairpins.

The experiment validated applicability of the DNA sensor in response to external force at a basic level. However, it is qualitative and suffers from manual error, making it highly difficult to reproduce. Therefore, we needed to modify the experimental procedure to apply a more controlled shear force. Experiments where networks of microtubules crosslinked with reversible DNA sensor were perturbed locally, in a controlled fashion, are described in the following subsection. 
(A)

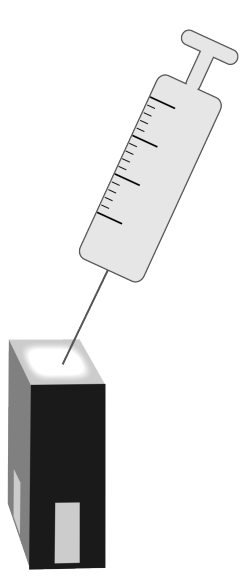

(B)

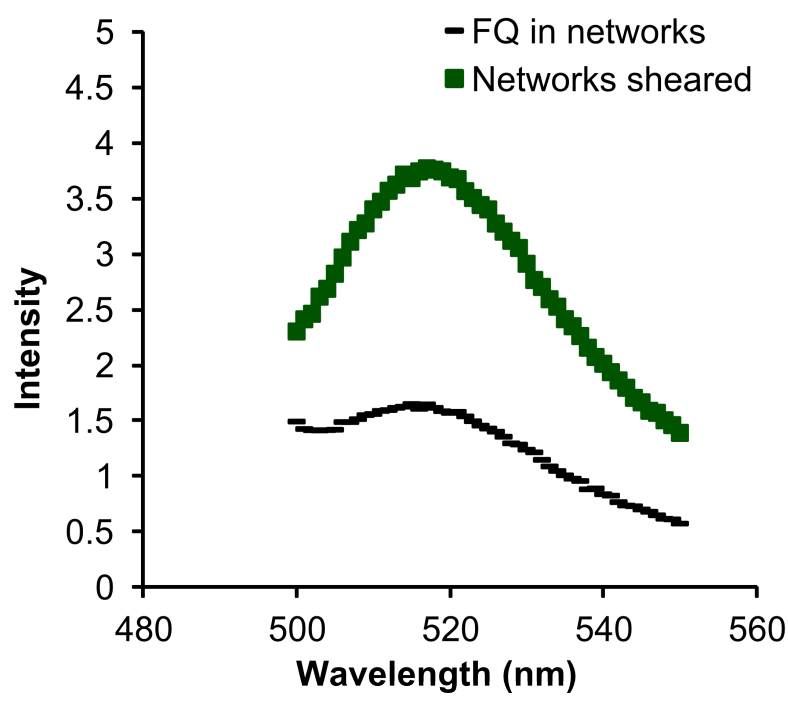

Figure 2.13: Bulk shear of microtubules crosslinked with DNA sensor. (A) Schematic of the experimental set-up, in which microtubules were crosslinked with DNA sensor in a cuvette and sheared with a needle. (B) Emission scan of fluorescence intensity from 500-550 $\mathrm{nm}$ (maximum emission $520 \mathrm{~nm}$ ) at an excitation wavelength of $494 \mathrm{~nm}$ was measured in a spectrophotometer. The fluorescence intensity increases after shearing the networks, most likely due to opening of DNA hairpins.

\subsubsection{Microscopic shear experiments}

Networks of microtubules crosslinked with the reversible DNA sensor were prepared in an open chamber attached to a coverslip. Experiments were performed on a home-built set-up explained in detail elsewhere. ${ }^{48}$ The set-up is equipped with an injection needle holder and a micro-injector (Inject man NI2; Eppendorf, Hamburg, Germany). A custom-pulled glass needle with a long, uniform and steady tapper was used for the experiments. The experimental set-up is shown in Fig. 2.14A. The needle is positioned just above the sample chamber and its movement can be manipulated with micrometer precision. The needle was lowered into chamber (position 1 ), displaced $30-40 \mu \mathrm{m}$ at a speed of $1000 \mu \mathrm{m} / \mathrm{s}$ (position 2) and then returned to original position.

Intensity of fluorescence from the recorded videos is plotted in Fig. 2.14B. A similar experiment was done in water as negative control and showed a constant background. Fluorescence intensity from experiments where the needle was held in a fixed position showed an initial steplike increase, corresponding to entry of the needle into the sample. The fluorescence intensity then decayed; corresponding to bleaching of the fluorophore. Back and forth movement of needle resulted in a saw-tooth pattern of fluorescence intensity. The observed saw-tooth pattern in intensity was in accordance with our expectations from opening and closing of the hairpins.

However, the fact that the fluorescence intensity never exceeded the initial background intensity on changing positions of needle was puzzling. Bleaching of fluorescence over duration of the experiment made it difficult to compare and analyze intensities at different positions (and times). We then performed an experiment to minimize bleaching of sample during the 
(A)

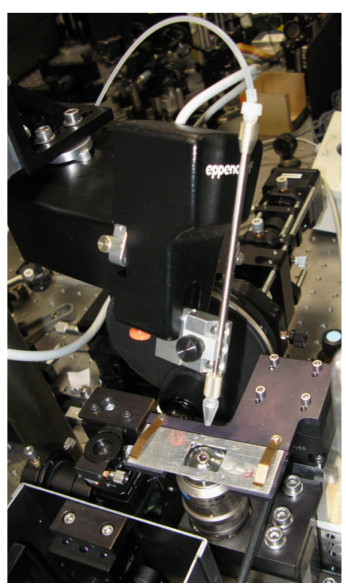

(B)

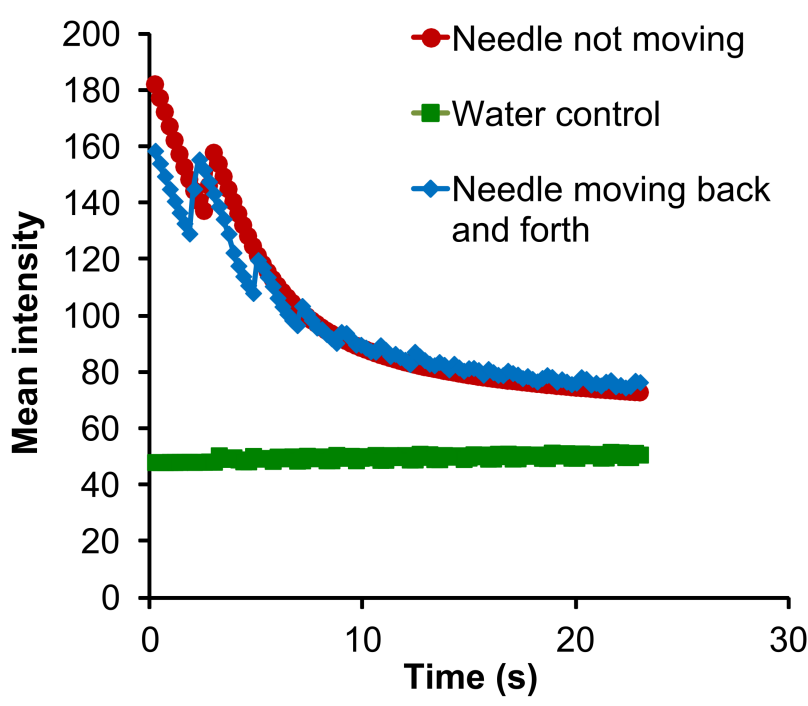

Figure 2.14: Microscopic shear of networks. (A) Experimental set-up showing the assembled position of glass needle used during the measurements. (B) Fluorescence intensity values from videos recorded while moving needle back and forth through networks of microtubules crosslinked with DNA sensor. Back and forth movement of needle results in a saw-tooth intensity pattern, which is not observed on maintaining a fixed position of the needle. Similar set of experiments performed in water, as a negative control, showed constant background intensity throughout the video.

(A)

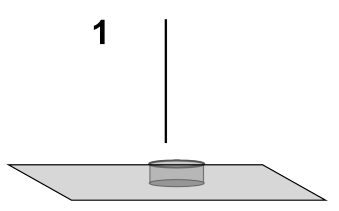

Not in contact

2

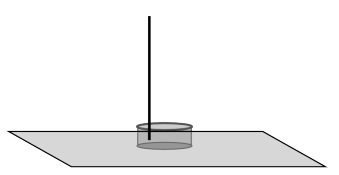

In position 1

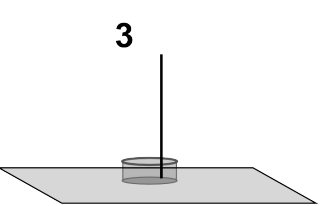

Move to position 2

4

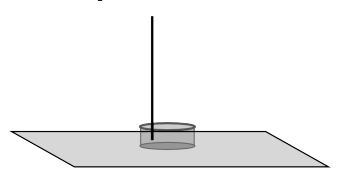

Back to position 1
(B)

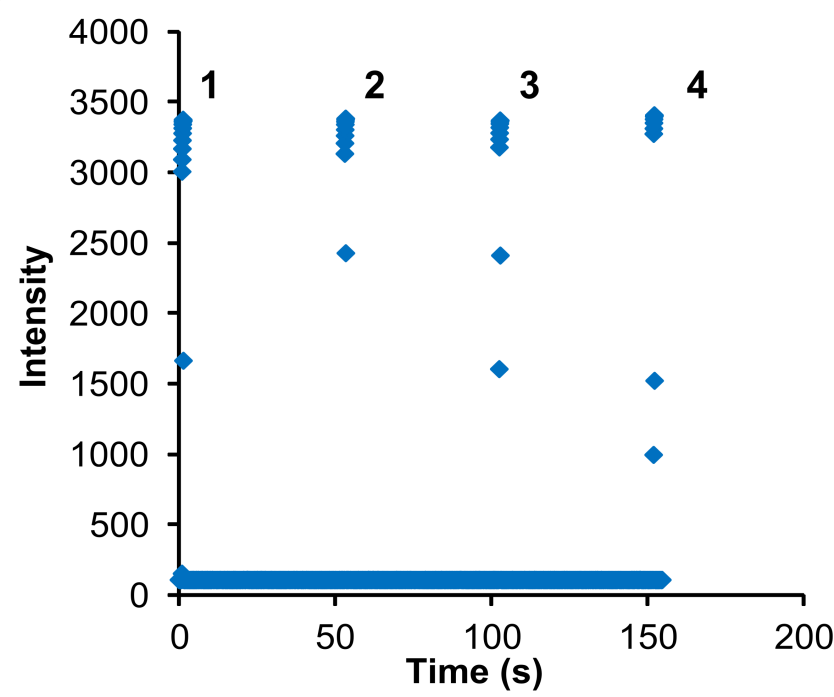

Figure 2.15: Experiments with minimized bleaching of fluorophores. (A) Schematic showing step-wise position of needle during the experiment. The numbers correspond to the chronological order of positions followed in the experiments. (B) Fluorescence intensity recorded by excitation of sample after every change in needle position. The intensities are same at each needle position, including the control where needle did not touch the sample. 
experiment. This was achieved by switching on the excitation laser intermittently, only after a change in position of the needle. A schematic showing step-wise change in position of needle during the experiment is shown in Fig. 2.15A. Analysis of the video showed that fluorescence intensities measured after moving the needle, indeed, never exceeded the background fluorescence intensity. The intensities at all needle positions were comparable, as shown in Fig. 2.15B.

Unfolding of the DNA hairpin must result in a higher fluorescence intensity than the quenched background, as shown in bulk control experiments (Section 2.5). What gives rise to the saw-tooth patterns in intensity, if not the unfolding of DNA sensor? Our speculation is that movement of the needle from position 1 to position 2 pulls the neighboring network into the field of view. Fluorophores in this part of network were not excited and therefore have not undergone bleaching. As a result, intensity of this part of network is higher than that measured from the field of view; the part that bleached due to excitation. On minimizing bleaching, we therefore measure a constant intensity, irrespective of position of the needle. Thus, we conclude that the observed saw-tooth intensity patterns give a misleading impression of opening of the sensor and are in reality artifacts from our shearing method.

The artifact from shear experiments with glass needle necessitated optimization of the experimental set-up for shearing networks. An alternative method of shearing could be perturbing the network using two beads in an optical trap. On one hand, we focus on developing better strategies for testing force sensing ability of our DNA sensor. On the other hand, it is also important to remember that our knowledge regarding our experimental system is limited. Mechanics of composite networks of crosslinked MTs have not been studied previously. Therefore, it was not only interesting but rather important for us to understand properties of these networks to customize our experiments accordingly. A simple example would be knowing mesh size of crosslinked networks of MTs, to choose the appropriate size of beads in force sensing experiments. Therefore, before resuming our experiments with force sensors, we focused on methods of crosslinking MTs with DNA and studied mechanical properties of these networks using rheology. A detailed description of the same is given in Chapter 3.

\section{Bibliography}

[1] Harris, A. K.; Wild, P.; Stopak, D. Science 1980, 208, 177-179.

[2] Alberts, B.; Bray, D.; Lewis, J.; Raff, M.; Roberts, K.; Watson, J. D.; Grimstone, A. Trends in Biochemical Sciences 1995, 20, 210-210.

[3] Dembo, M.; Wang, Y.-L. Biophysical journal 1999, 76, 2307-2316.

[4] Gaudet, C.; Marganski, W. A.; Kim, S.; Brown, C. T.; Gunderia, V.; Dembo, M.; Wong, J. Y. Biophysical journal 2003, 85, 3329-3335.

[5] Balaban, N. Q.; Schwarz, U. S.; Riveline, D.; Goichberg, P.; Tzur, G.; Sabanay, I.; Mahalu, D.; Safran, S.; Bershadsky, A.; Addadi, L.; Geiger, B. Nature cell biology 2001, 3, 466-472. 
[6] Tan, J. L.; Tien, J.; Pirone, D. M.; Gray, D. S.; Bhadriraju, K.; Chen, C. S. Proceedings of the National Academy of Sciences 2003, 100, 1484-1489.

[7] Grashoff, C.; Hoffman, B. D.; Brenner, M. D.; Zhou, R.; Parsons, M.; Yang, M. T.; McLean, M. A.; Sligar, S. G.; Chen, C. S.; Ha, T.; Schwartz, M. A. Nature 2010, 466, 263-266.

[8] Meng, F.; Suchyna, T. M.; Sachs, F. FEBS journal 2008, 275, 3072-3087.

[9] Meng, F.; Sachs, F. Journal of cell science 2011, 124, 261-269.

[10] Morimatsu, M.; Mekhdjian, A. H.; Adhikari, A. S.; Dunn, A. R. Nano letters 2013, 13, 3985-3989.

[11] Wang, X.; Ha, T. Science 2013, 340, 991-994.

[12] Blakely, B. L.; Dumelin, C. E.; Trappmann, B.; McGregor, L. M.; Choi, C. K.; Anthony, P. C.; Duesterberg, V. K.; Baker, B. M.; Block, S. M.; Liu, D. R.; Chen, C. S. Nature methods 2014, 11, 1229-1232.

[13] Zhang, Y.; Ge, C.; Zhu, C.; Salaita, K. Nature communications 2014, 5.

[14] Mogilner, A.; Oster, G. Biophysical journal 2003, 84, 1591-1605.

[15] Bershitsky, S. Y.; Tsaturyan, A. K.; Bershitskaya, O. N.; Mashanov, G. I.; Brown, P.; Burns, R.; Ferenczi, M. A. Nature 1997, 388, 186-190.

[16] Schutt, C. E.; Lindberg, U. Proceedings of the National Academy of Sciences 1992, 89, 319-323.

[17] Abraham, V. C.; Krishnamurthi, V.; Taylor, D. L.; Lanni, F. Biophysical journal 1999, 77, 1721-1732.

[18] Du Roure, O.; Saez, A.; Buguin, A.; Austin, R. H.; Chavrier, P.; Siberzan, P.; Ladoux, B. Proceedings of the National Academy of Sciences of the United States of America 2005, 102, 2390-2395.

[19] Dumont, S.; Mitchison, T. J. Current Biology 2009, 19, R749-R761.

[20] Watson, J.; Crick, F. Nature 1953, 421, 397-3988.

[21] Doi, M.; Edwards, S. F. The theory of polymer dynamics; Clarendon Press Oxford, 1986; Vol. 222.

[22] Bustamante, C.; Smith, S. B.; Liphardt, J.; Smith, D. Current opinion in structural biology 2000, 10, 279-285.

[23] Essevaz-Roulet, B.; Bockelmann, U.; Heslot, F. Proceedings of the National Academy of Sciences 1997, 94, 11935-11940. 
[24] Bath, J.; Turberfield, A. J. Nature nanotechnology 2007, 2, 275-284.

[25] Saccà, B.; Niemeyer, C. M. Angewandte Chemie International Edition 2012, 51, 58-66.

[26] Rothemund, P. W. Nature 2006, 440, 297-302.

[27] Goodman, R. P.; Schaap, I. A.; Tardin, C. F.; Erben, C. M.; Berry, R. M.; Schmidt, C. F.; Turberfield, A. J. Science 2005, 310, 1661-1665.

[28] Grange, W.; Strunz, T.; Schumakovitch, I.; Guntherodt, H.; Hegner, M. Single Molecules 2001, 2, 75-78.

[29] Schumakovitch, I.; Grange, W.; Strunz, T.; Bertoncini, P.; Güntherodt, H.-J.; Hegner, M. Biophysical journal 2002, 82, 517-521.

[30] Lee, G. U.; Chrisey, L. A.; Colton, R. J. Science 1994, 266, 771-773.

[31] Strunz, T.; Oroszlan, K.; Schäfer, R.; Güntherodt, H.-J. Proceedings of the National Academy of Sciences 1999, 96, 11277-11282.

[32] Hatch, K.; Danilowicz, C.; Coljee, V.; Prentiss, M. Physical Review E 2008, 78, 011920.

[33] Walsh, A. S.; Yin, H.; Erben, C. M.; Wood, M. J.; Turberfield, A. J. ACS nano 2011, 5, 5427-5432.

[34] Förster, T. Annalen der physik 1948, 437, 55-75.

[35] Le Reste, L.; Hohlbein, J.; Gryte, K.; Kapanidis, A. N. Biophysical journal 2012, 102, 2658-2668.

[36] Jares-Erijman, E. A.; Jovin, T. M. Nature biotechnology 2003, 21, 1387-1395.

[37] Roy, R.; Hohng, S.; Ha, T. Nature methods 2008, 5, 507-516.

[38] Zadeh, J. N.; Steenberg, C. D.; Bois, J. S.; Wolfe, B. R.; Pierce, M. B.; Khan, A. R.; Dirks, R. M.; Pierce, N. A. Journal of computational chemistry 2011, 32, 170-173.

[39] Renner, S.; Bessonov, A.; Gerland, U.; Simmel, F. C. Journal of Physics: Condensed Matter 2010, 22, 454119.

[40] Woodside, M. T.; Anthony, P. C.; Behnke-Parks, W. M.; Larizadeh, K.; Herschlag, D.; Block, S. M. Science 2006, 314, 1001-1004.

[41] Woodside, M. T.; Behnke-Parks, W. M.; Larizadeh, K.; Travers, K.; Herschlag, D.; Block, S. M. Proceedings of the National Academy of Sciences 2006, 103, 6190-6195.

[42] Neish, C. S.; Martin, I. L.; Henderson, R. M.; Edwardson, J. M. British journal of pharmacology 2002, 135, 1943-1950. 
[43] Kong, F.; García, A. J.; Mould, A. P.; Humphries, M. J.; Zhu, C. The Journal of cell biology 2009, 185, 1275-1284.

[44] Yuan, C.; Chen, A.; Kolb, P.; Moy, V. T. Biochemistry 2000, 39, 10219-10223.

[45] Moy, V. T.; Florin, E.-L.; Gaub, H. E. Colloids and Surfaces A: Physicochemical and Engineering Aspects 1994, 93, 343-348.

[46] Bodensiek, K. Combining force and fluorescence microscopy for the manipulation and detection of single cells, viruses, and proteins. Ph.D. thesis, Georg August University, Göttingen, 2014.

[47] Gardel, M.; Nakamura, F.; Hartwig, J.; Crocker, J.; Stossel, T.; Weitz, D. Proceedings of the National Academy of Sciences of the United States of America 2006, 103, 1762-1767.

[48] Wessel, A. D. Intracellular micromechanics of the syncytial Drosophila embryo. Ph.D. thesis, Georg August University, Göttingen, 2015. 


\section{Chapter 3}

\section{Rheology}

Rheology is the study of deformation and flow in materials under an externally applied force. In practice, rheology is divided into two branches - microrheology and macrorheology. Microrheology involves study of local deformations in samples, for example, using an optical trap. Macrorheology is used to characterize the mechanical response of bulk samples to an external force. We have used bulk rheology for characterizing mechanical behavior of networks in all our experiments. A detailed description of rheology and methods is given in this chapter. 


\subsection{Rheology of elastic and viscous materials}

Rheology is the characterization of the mechanical properties of materials under an applied deformation. The simplest types of materials are solid-like elastic materials or viscous fluids. We begin with the description of purely elastic and viscous materials and then move on to materials exhibiting complex viscoelastic behavior, more common in biological systems.

Consider a simple case of a purely elastic box of given height $H$ and area $A$. The top of the box is sheared with an external force $F$, resulting in a displacement of length $D$ as shown in Fig. 3.1A. The resultant physical quantities of shear stress and strain are defined as $\sigma=F / A$ and $\gamma=D / H$, respectively. An ideal elastic material can be compared mechanically to a Hookean spring. The stress and strain are related through a material parameter called shear modulus $G$, given by

$$
G=\frac{\sigma}{\gamma}
$$

The shear modulus here describes stiffness of a material.

The behavior of a viscous material is very different from an elastic material. Fig. $3.1 \mathrm{~B}$ shows a viscous material enclosed between two plates separated by height $H$. Here, shearing the top plate with a velocity $V$ generates a flow in the fluid. The fluid layers move at different velocities; the top layer moves with a maximum velocity $V$ while the bottom layer remains stationary. As the flow induces a continuous increase in strain, the rate of change in strain becomes the main factor that determines drag forces in the fluid. The shear rate $\dot{\gamma}$, for a Newtonian fluid, is constant and is given by the velocity gradient $V / H$ between the plates. The stress and shear rate are related through a material parameter called viscosity $\eta$, given by

$$
\sigma=\eta \dot{\gamma}
$$

Viscosity is a material property that describes its resistance to flow.

Experimentally, the mechanical response of materials can be tested using transient or dynamic tests. In a transient measurement, an external deformation (stress or strain) is applied for a finite time and the response of the material (strain or stress) is measured over a certain time. An example of one such type of measurement is the creep test. In a creep experiment, a stress is imposed on a sample and the resultant strain is measured over time, $t_{1}$. The material is then relieved of the imposed stress and allowed to recover while monitoring the strain over relaxation time, $t_{2}$. Fig. 3.1C, D shows differences in the response of elastic and viscous materials to an external stress. An elastic material shows an instant increase in strain on applying a stress and an instant recovery on stress relaxation, as shown in Fig. 3.1 C. On the other hand, deformation of a purely viscous material is permanent and does not recover on stress relaxation, as shown in Fig. 3.1D. Dynamic measurements are oscillatory measurements in which a sinusoidal strain, $\gamma(t)=\gamma_{0} \sin (\omega t)$, is applied to the sample and the resultant stress response is measured. Fig. 3.1E shows the measured stress for an elastic material, which is exactly in phase with the strain. On the contrary, stress response for a viscous material is exactly out of phase with an angle of $\pi / 2$, as shown in Fig. 3.1F. 
(A)
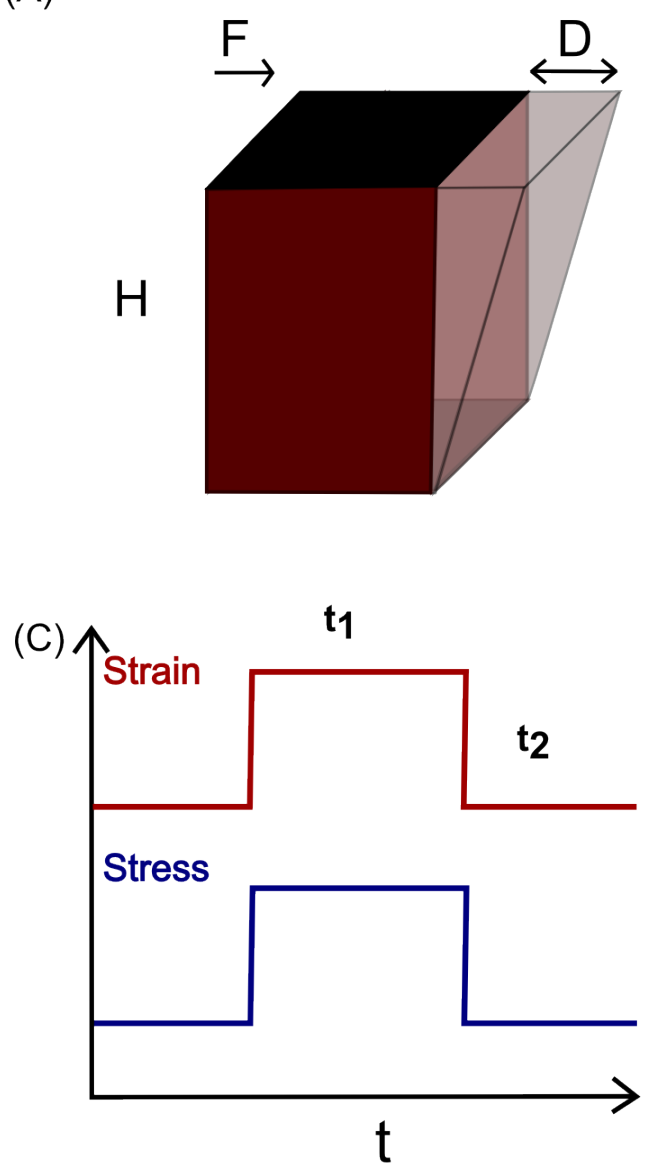

(E)

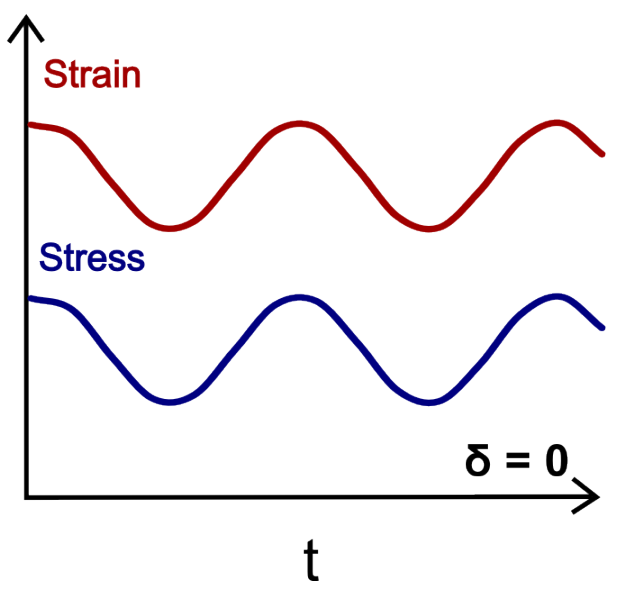

(B)

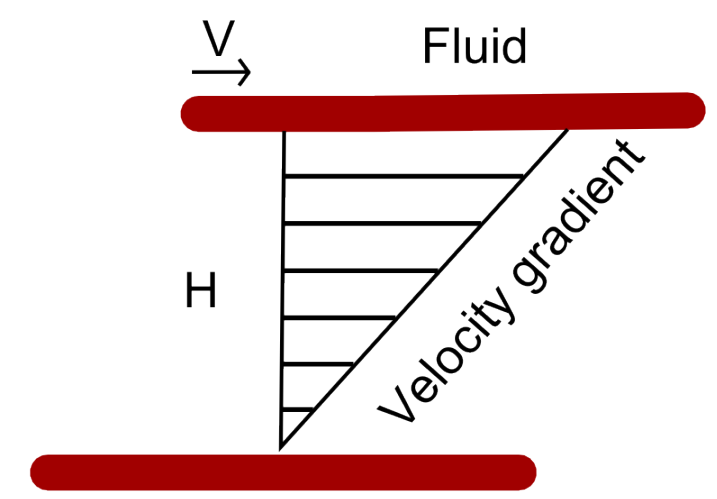

(D)

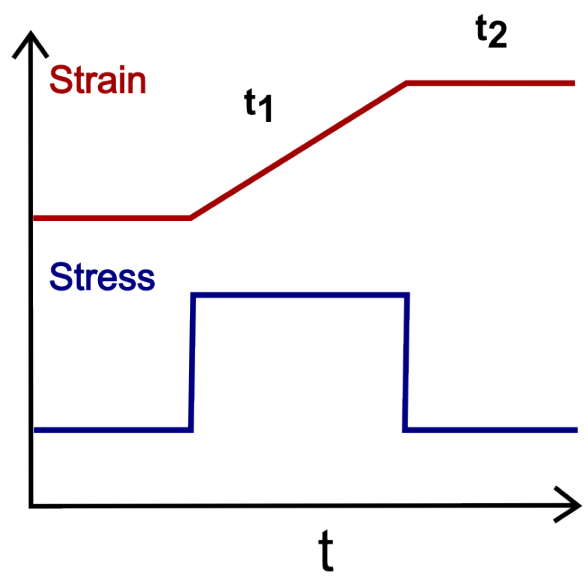

(F)

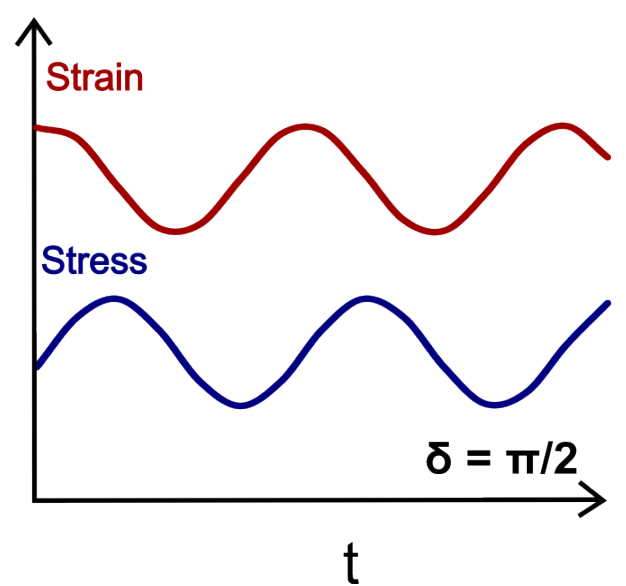

Figure 3.1: Rheology of elastic and viscous materials. (A) An elastic box of height $H$ and area $A$ is sheared with a force $F$ such that displacement is $D$. The strain and stress are given by $\gamma=D / H$ and $\sigma=F / A$, respectively. (B) Shearing a fluid results in a velocity gradient across fluid layers, given by $V / H$, where $V$ is velocity of flow in chamber of height $H$. (C) In a purely elastic material, the strain increases and relaxes instantly on application and removal of external stress. (D) In case of a viscous material, strain increases slowly at a constant rate on stress application and remains constant even after stress relaxation. (E) Stress response on imposing a sinusoidal strain on an elastic material is in phase with the strain and $(\mathrm{F})$ exactly out of phase $(\delta=\pi / 2)$ for a viscous material. 
The mechanical behavior of purely elastic and purely viscous materials is simple to characterize and predict. Many materials, however, cannot be categorized as purely elastic or viscous but are rather a combination of both. Models for understanding mechanical properties of such viscoelastic materials are described in the following section.

\subsection{Linear viscoelasticity}

Viscoelastic materials show a combination of elastic and viscous behavior. We saw that elastic materials can be completely described by the physical quantities of stress and strain. Similarly, Newtonian fluids are characterized simply by describing stress and shear rate. However, viscoelastic materials require consideration of an additional variable - time. The rheological properties of soft, viscoelastic materials may depend on the time scale at which they are probed.

One way of describing the behavior of viscoelastic materials is using a combination of mechanical elements of a spring and a dashpot. Two simple models that are commonly used to describe linear viscoelastic behavior of materials are Maxwell and Kelvin-Voigt models.

\subsubsection{Maxwell model}

The Maxwell model describes a viscoelastic material as a spring and a dashpot connected in series, as shown in Fig. 3.2A. The stress across the spring and the dashpot is constant. The total strain is additive of the individual strain experienced by the spring and the dashpot. The strain response of a Maxwell material on subjecting it to a creep test is qualitatively shown in Fig. 3.2. C. On application of stress at $t_{1}$, the spring deforms instantly, giving a sharp increase in strain. The viscous part shows a delayed response and then flows with a constant rate. Relaxation of stress at $t_{2}$, results in an instantaneous relaxation of the spring resulting in a small decrease in strain. The viscous part does not show a tendency to recover, thereby maintaining a permanent strain. The Maxwell model, therefore, describes a viscoelastic liquid showing plastic deformation.

\subsubsection{Kelvin-Voigt model}

An alternative model is the Kelvin Voigt model, which assumes a spring and a dashpot to be connected in parallel, as shown in Fig. 3.2 B. In this model, the strain experienced by the spring and the dashpot is same. Fig. 3.2D shows creep response of a Kelvin-Voigt material. When the material is subjected to a stress at $t_{1}$, the response of the spring is delayed by the dashpot, resulting in slow creep of the strain. Similarly, relaxation of stress at $t_{2}$ results in a delayed but complete recovery of strain. Thus, the Kelvin-Voigt model describes behavior of a viscoelastic solid.

The Maxwell and Kevin-Voigt models are basic and useful for the purpose of understanding viscoelastic behavior. However, biological systems are, in general, complex and cannot be described simply by single spring and dashpot models. There are many other complicated 
(A)

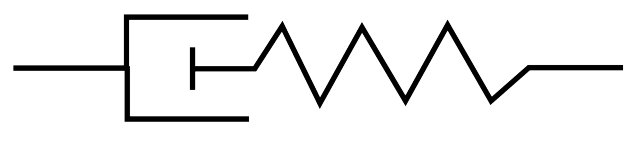

(C)

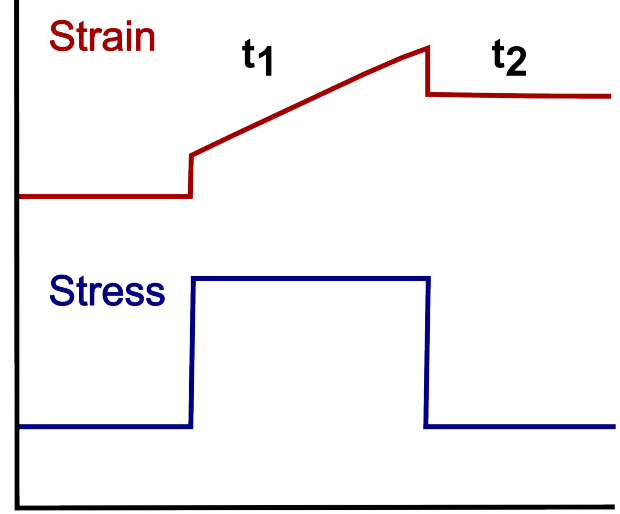

(B)

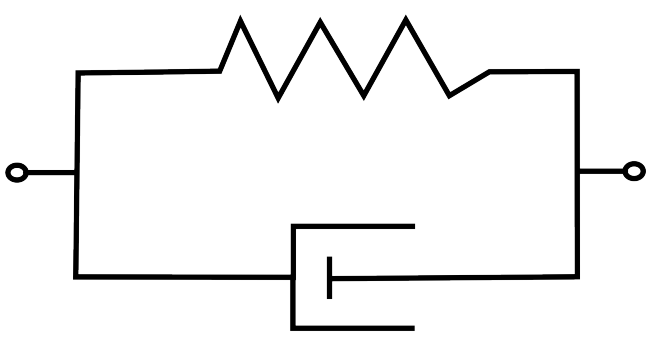

(D)

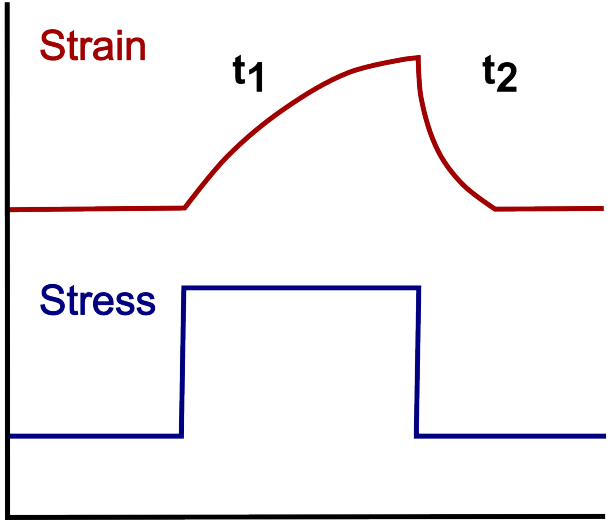

Figure 3.2: Models for viscoelastic materials. (A) Maxwell model describes a viscoelastic material as a spring and a dashpot connected in series. (B) Kelvin-Voigt model describes a viscoelastic material as a spring and a dashpot connected in parallel. (C) Creep test on Maxwell material shows an initial instantaneous increase in strain on stress application $\left(t_{1}\right)$, given by the spring. The dashpot then results in flow of the material at a constant rate. Relaxation of stress $\left(t_{2}\right)$ gives an instant relaxation of the spring, resulting in a sharp drop in strain. The dashpot, however, does not relax, resulting in a permanent strain. (D) Stress application $\left(t_{1}\right)$ on Kelvin-Voigt material results in a slow creep of the strain, due to damping of the spring by the dashpot. The recovery of strain is delayed but complete on relaxation of stress $\left(t_{2}\right)$.

models that use combinations of multiple springs and dashpots to describe viscoelastic materials more accurately. 1 A generalized approach for understanding viscoelastic behavior is based on the Boltzmann superposition principle, which is elaborated below.

\subsubsection{Boltzmann superposition principle}

The concept of Boltzmann superposition principle is that the material retains 'memory' of deformations applied to it in the past. Also, the total deformation is additive of all previous deformations. This can be explained as follows. If a material is subjected to a strain $\gamma$ at a certain time $t$, then the stress relaxation over time is given by,

$$
\sigma(t)=G(t) \gamma
$$


where $G$ is the relaxation modulus. Suppose that a series of strains $\gamma_{1}, \gamma_{2}, \gamma_{3}$ have been imposed on a system at times $t_{1}, t_{2}$ and $t_{3}$, respectively. Then according to the Boltzmann superposition principle, the stress $\sigma(t)$ can be obtained as a sum of all previous stresses on the material as follows:

$$
\sigma(t)=G\left(t-t_{1}\right) \gamma_{1}+G\left(t-t_{2}\right) \gamma_{2}+G\left(t-t_{2}\right) \gamma_{3}
$$

Passing to the continuous time limit, where $\gamma=\gamma(t)$, one obtains the following stress strain relation:

$$
\sigma(t)=\int_{-\infty}^{t} G\left(t-t^{\prime}\right) \gamma\left(t^{\prime}\right) d t^{\prime}
$$

The equation above takes into account different relaxation time scales in a system. It holds for transient as well as dynamic measurements. In dynamic, oscillatory experiments, measuring stress response to an imposed strain determines the shear modulus. The shear modulus has two components. One is the storage modulus $G^{\prime}$, given by the elastic part of the system. Another one is the loss modulus $G^{\prime \prime}$, which represents viscous component of the system. Oscillatory measurements are described in detail in the following section.

It is important to remember, in general, that the models described above are applicable for linear viscoelastic response. Therefore, they can only be useful at low deformations, such that the stress-strain relation remains linear.

\subsection{Rheometer}

(A)

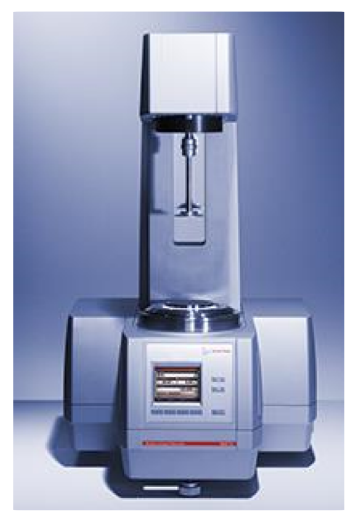

(B)

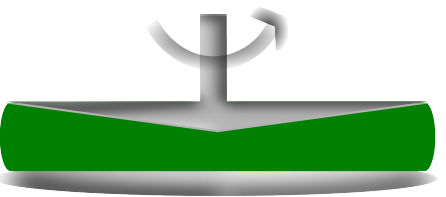

(C)

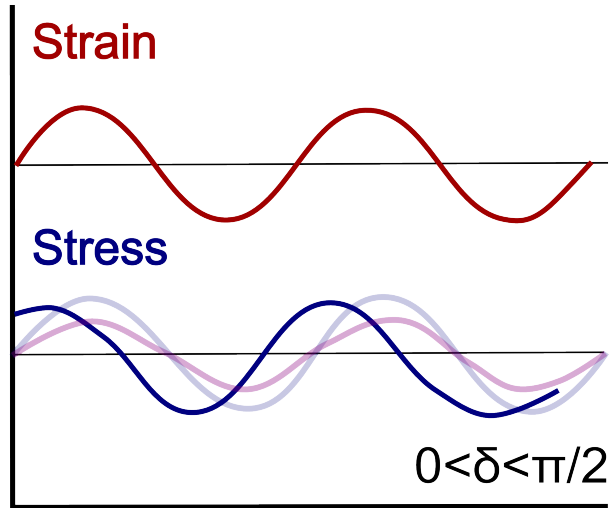

Figure 3.3: Rheometer and oscillatory test. (A) Anton Paar rheometer MCR series (Image from www.anton-paar.com).

(B) Schematic of a cone plate. (C) Oscillatory tests for viscoelastic materials show a phase shift $(\delta)$ with, both, in phase (dark blue) and out of phase (faded colors) contributions. 
The instrument used for measuring material properties in bulk solutions is called a rheometer. We used a commercial rheometer (Physica MCR 501, Anton Paar, Ostfildern, Germany) in our experiments. The rheometer is equipped with a fixed bottom plate and a movable top plate. The bottom plate is made of chromium oxide; temperature controlled by a Peltier element. The top plate is made of stainless steel and can either be a parallel plate or a cone with a small angle, typically $1^{\circ}$ or $2^{\circ}$. An image of the rheometer and schematic of a cone plate are shown in Fig. 3.3A, B, respectively. The distinguishing features of each of the plates are summarized in Table 3.1.

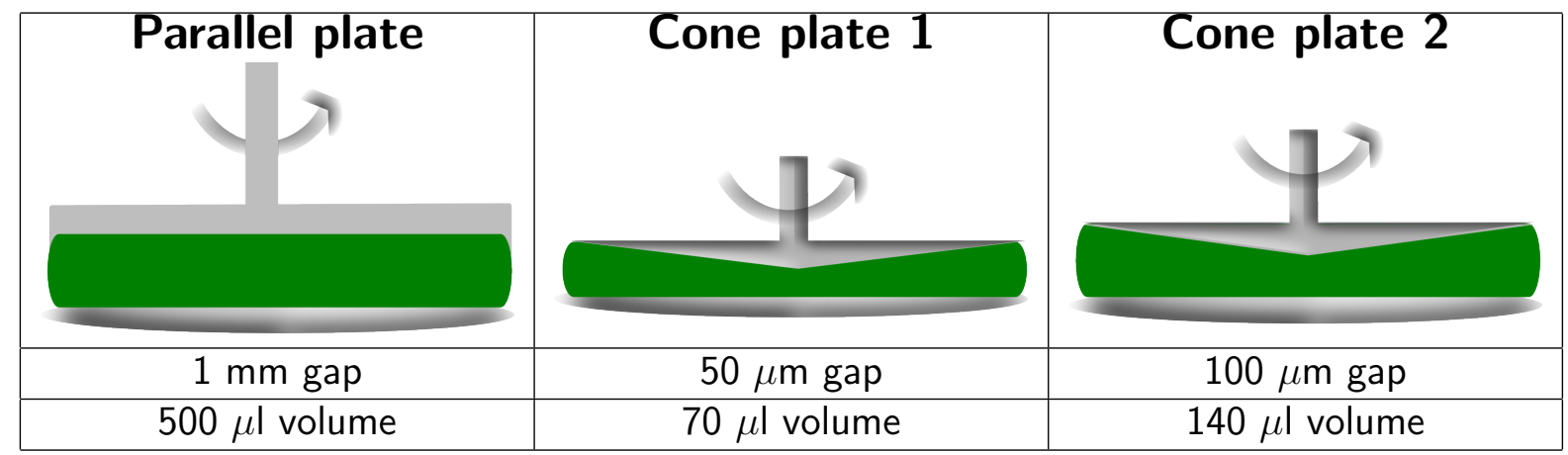

Table 3.1: Rheometer plates. Distinguishing features of different types of top plates of the rheometer are given.

The main advantage of a cone plate over a parallel plate is that it maintains a constant shear rate across the sample. Each of the cone plates has a different gap size, corresponding to truncation of the cone. It follows that the volume requirements for different cone plates will also be different. It has been shown that smaller cone angles result in relatively large errors in gap size and thereby in calculated strain and strain rate values. ${ }^{[2}$ However, sample volume becomes a limiting parameter, especially for high concentrations of biological materials. Therefore, we used a cone plate with an angle of $1^{\circ}(\mathrm{CP}-1)$ and $25 \mathrm{~mm}$ diameter in our experiments, as it requires a small sample volume of $70-80 \mu \mathrm{l}$. All measurements were performed at $23^{\circ} \mathrm{C}$. The sample was surrounded by wet paper towels to minimize evaporation.

\subsubsection{Linear viscoelastic measurements}

The elastic and viscous contributions of materials at different time scales can be extracted using oscillatory tests in a rheometer. The top plate applies a sinusoidal strain, $\gamma(t)=\gamma_{0} \sin (\omega t)$, and the resultant stress is measured. The stress response in case of viscoelastic materials, shows in phase as well as out of phase contributions $(0<\delta<\pi / 2)$, as shown in Fig. 3.3. C. From Eq. (3.2.3), the stress response to the imposed sinusoidal strain can be written as

$$
\sigma=G^{\prime}(\omega) \gamma_{0} \sin (\omega t)+G^{\prime \prime}(\omega) \gamma_{0} \cos (\omega t)
$$


As mentioned above, $G^{\prime}$ is the storage modulus that represents the elastic component or energy stored in the system. $G^{\prime \prime}$ is the loss modulus that gives the energy dissipated due to viscous contribution.
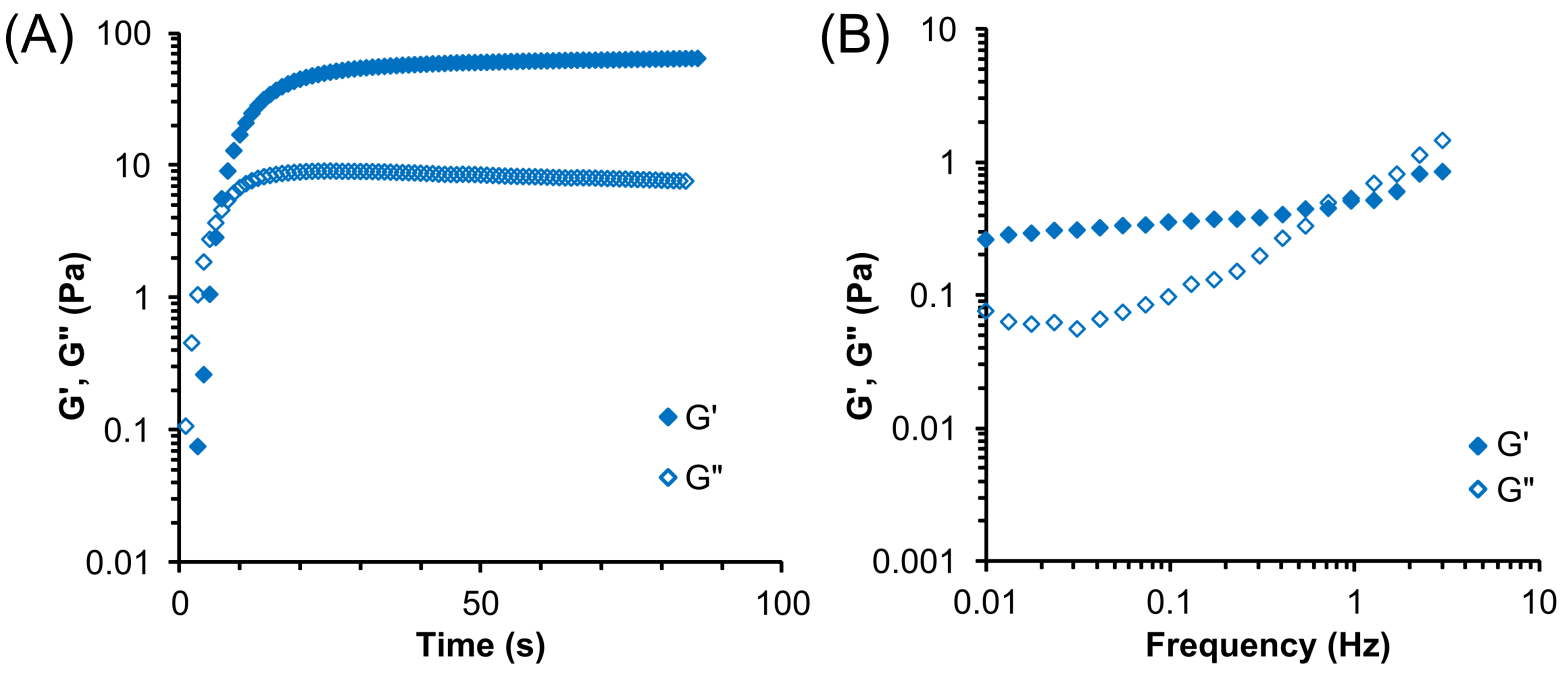

Figure 3.4: Linear rheology tests. (A) Time sweep showing polymerization kinetics of a polyacrylamide gel. (B) Frequency sweep of actin filaments $(1 \mathrm{mg} / \mathrm{ml})$ shows weak dependence of $G^{\prime}$ on frequency. Crossover of $G^{\prime \prime}$ over $G^{\prime}$ around $1 \mathrm{~Hz}$ indicates fluid-like behavior of actin at higher frequencies.

Oscillatory measurements are done to obtain linear viscoelastic (LVE) response of materials at small deformations. There are three types of LVE measurements - time sweep, frequency sweep and amplitude sweep. As the names suggest, these tests measure mechanical properties with time, frequency or amplitude as variables. Time sweep experiments are useful for characterizing polymerization dynamics of samples such as polyacrylamide gels, as shown in Fig. 3.4A. Measuring mechanical properties over a range of frequencies gives important information regarding the time dependent mechanical behavior of a material. Fig. 3.4B shows an example of a frequency sweep on polymerized actin $(1 \mathrm{mg} / \mathrm{ml}) . G^{\prime}$ is higher than $G^{\prime \prime}$ at lower frequencies. Above approximately $1 \mathrm{~Hz}$, we see a crossover regime after which $G^{\prime \prime}$ dominates over $G^{\prime}$. This means that actin shows fluid-like behavior at higher frequencies. An amplitude sweep is useful in determining yield point of a material as well as for characterizing strain stiffening or softening behavior. Strain stiffening, for example, is a commonly observed property in tissues as a protection mechanism against damage from excessive strain. $\frac{3}{3}$ However, one must be careful with such measurements as some materials may show nonlinear behavior at large amplitudes. As the material response is assumed to be in linear range by the rheometer software, these simple tests cannot be used for characterizing nonlinearity in a system. 


\subsubsection{Nonlinear tests}

Many types of artificial as well as biological samples show similar linear response but a diverse, informative nonlinear regime. $\frac{465}{4}$ Nonlinearity in systems can be characterized using specialized tests such as large amplitude oscillatory shear (LAOS), strain ramp and differential measurements. We used differential measurements in our experiments, in which a stress, also called prestress, is imposed on the sample by rotation of the top plate of the rheometer. A small oscillatory stress, typically $10 \%$ of the imposed prestress, is superimposed on top of the rotational stress. The strain response to this small oscillatory stress is linear and gives a differential modulus, $K^{\prime}$, written as

$$
K^{\prime}=\frac{d \sigma}{d \gamma} .
$$

The role of differential measurements or other nonlinear tests in the field of network rheology is summarized in the next section.

\subsection{Mechanics of biopolymer networks}

Elasticity of living cells has been measured to be around $1000 \mathrm{~Pa}$ from various methods such as AFM and microrheology methods. ${ }^{6-8]}$ However, cells are active systems that tune their elasticity according to the substrate; most likely by endogenous forces exerted by molecular motors. ${ }^{9} \cdot 11$ This makes it difficult to interpret the origin and exact nature of measured cellular elasticity.

Cellular rigidity is mediated by bundles of crosslinked actin in cortex. ${ }^{12 \mid 13} \mathrm{In}$ fact, disruption of polymeric actin has shown to cause softening of cells. 14115 Therefore, mechanics of in vitro actin networks has been widely studied as a bottom-up approach in understanding cell mechanics. $\frac{16-18}{-18}$ Network behavior has been described using combined biophysical approaches of experimental rheology, theoretical modeling and simulations.

Experiments have been done on networks of entangled actin and actin crosslinked with a wide range of biologically relevant actin binding proteins (ABPs). $\frac{[19}{[19}$ The elasticity $G^{\prime}$ of these networks is entropic in origin, i.e., the restoring force appears due to reduction in number of conformations on application of external deformation. ${ }^{20}$ The network microstructure and mechanics are largely governed by concentrations of actin and crosslinker, as well as compliance of the crosslinker. ${ }^{21}$ For instance, densely crosslinked networks give rise to bundles of actin instead of isotropic networks that are observed with weak crosslinking. ${ }^{22123}$ The high crosslinker density networks exhibit nonlinear stiffening behavior above a critical stress applied in differential measurements. ${ }^{19124-26}$ The observed scaling exponent of differential modulus $K^{\prime}$ over stress covers a broad range from 0.1-2 depending on the type of ABP used for crosslinking. 19/24/27/28

Compliance of the crosslinks especially influences critical crosslinker concentrations required for network formation. Actin crosslinked with filamin, a compliant ABP, can withstand about 1000-fold larger stresses in comparison to networks with stiff crosslinkers of comparable concentration. ${ }^{[5}$ In the nonlinear regime, the differential modulus scales linearly with stress $\left(K^{\prime} \sim \sigma\right)$; similar to scaling laws observed with living cells. ${ }^{6 / 29}$ Indeed, networks of bundled actin and 
compliant crosslinker structurally mimic the cellular cortex where stiff actin stress fibres are embedded in a soft actin mesh.

Thus, composite networks of stiff rods and flexible crosslinkers provide a generalized system to describe cortical networks. The first theoretical model describing such networks was given in Ref. 30. The model assumes rods to be embedded in an effective medium and predicts a scaling of $K^{\prime} \sim \sigma$, that fit well with in vitro and in vivo experimental data, as described above.

However, there are certain shortcomings of existing experimental systems as well as theoretical models. On the experimental side, it is difficult to fine tune parameters in composite actin networks. One problem is that increasing concentration of crosslinkers will not solely increase the crosslink density but also increase filament bundling and thereby change persistence length of filaments. Secondly, possible variations in length of crosslinker are limited due to maximum length of binding proteins. Therefore, we need an ideal model system with tunable parameters to mimic composite cellular networks.

On the theoretical front, the main problem with modeling is in the assumption of an effective medium. The assumption implies that the medium is self consistent and can be subjected to an infinite strain. However, in a composite network of infinitely rigid filaments and compliant crosslinkers, any applied strain is borne by the crosslinkers alone. Since the crosslinkers cannot be subjected to infinite strain owing to the force divergence at a finite extension, the network as a whole cannot withstand infinite strain. Recently, a fresh theoretical approach has been applied to model these composite networks. ${ }^{31132}$ Within this approach, the shortcomings of the previous model are resolved. One of the major predictions, in contrast to the previous theory, is scaling of differential modulus, given by $K^{\prime} \sim \sigma^{3 / 2}$ instead of $K^{\prime} \sim \sigma$.

Experimentally, an ideal system of composite networks can be achieved by crosslinking microtubules with DNA. DNA is a flexible polymer (persistence length, $L_{p} \sim n m$ ), monodisperse with precise length tunability; an ideal crosslinker. Microtubules, in contrast, are stiff biopolymers $\left(L_{p} \sim m m\right)$ that can be polymerized and stabilized for in vitro experiments. $\frac{33134}{\text { These }}$ heterogeneous networks are, therefore, an optimum model system of stiff rods and flexible crosslinkers for comparison with theory and simulations.

Additionally, network behavior of crosslinked microtubules has not been as well characterized, in comparison to actin. Moreover, the existing experiments on microtubule networks have mainly been done with rigid crosslinkers. ${ }^{35136}$ Therefore, crosslinking microtubules with compliant DNA that can be tuned in length is a new and interesting system to investigate. Lastly, we aim to attach our DNA sensor to cytoskeletal filaments in cells. Therefore, crosslinking of microtubules with DNA sensor in vitro is a step forward in that direction. The following sections describe our experimental approaches in crosslinking microtubules (MTs) with DNA and mechanics of crosslinked networks.

\subsection{Crosslinking microtubules with DNA}

The choice of an intermediate crosslinker for linking DNA to microtubules is an important step in our experiments. The crosslinker should satisfy certain conditions for an optimum network assembly. Most importantly, it must attach specifically to the biopolymers but at the same 
time have minimum crossreactions. For instance, crosslinking biotinylated DNA and biotinylated microtubules via streptavidin would follow a simple crosslinking mechanism. However, this would also result in unwanted rigid crosslinks by interaction of biotinylated microtubules through streptavidin. This is highly undesirable as we aim to study mechanics of composite networks of MTs crosslinked with complaint DNA crosslinkers. Needless to say, presence of the crosslinker, by itself, should not contribute to network mechanics. Also, investigating a new system is best achieved by minimizing variables in the system. Therefore, we avoid transient crosslinkers, such as molecular motors, to have permanent crosslinking and eliminate bindingunbinding effects from our networks. Bearing all these desirable features in mind, we chose a commercially available chemical heterobifunctional crosslinker, sulfo-SMCC, for our experiments. Sulfo-SMCC (Sulfosuccinimidyl 4-(N-maleimidomethyl)cyclohexane-1-carboxylate) has a N-hydroxysuccinimide (NHS) ester on one end to react with primary amines. On the other end, it consists of a maleimide group that reacts specifically with sulfhydryl/thiol (SH) groups. In our experiments, maleimide end of sulfo-SMCC was linked to thiol modified ends of DNA and NHS ester group to primary amines present on microtubules. Crossreaction of maleimide of sulfo-SMCC with thiols on microtubules was avoided by blocking them with maleimide, prior to incubation with the crosslinker. The crosslinker is very small ( $8.3 \AA$ ) and should not contribute largely to mechanical properties of the networks.

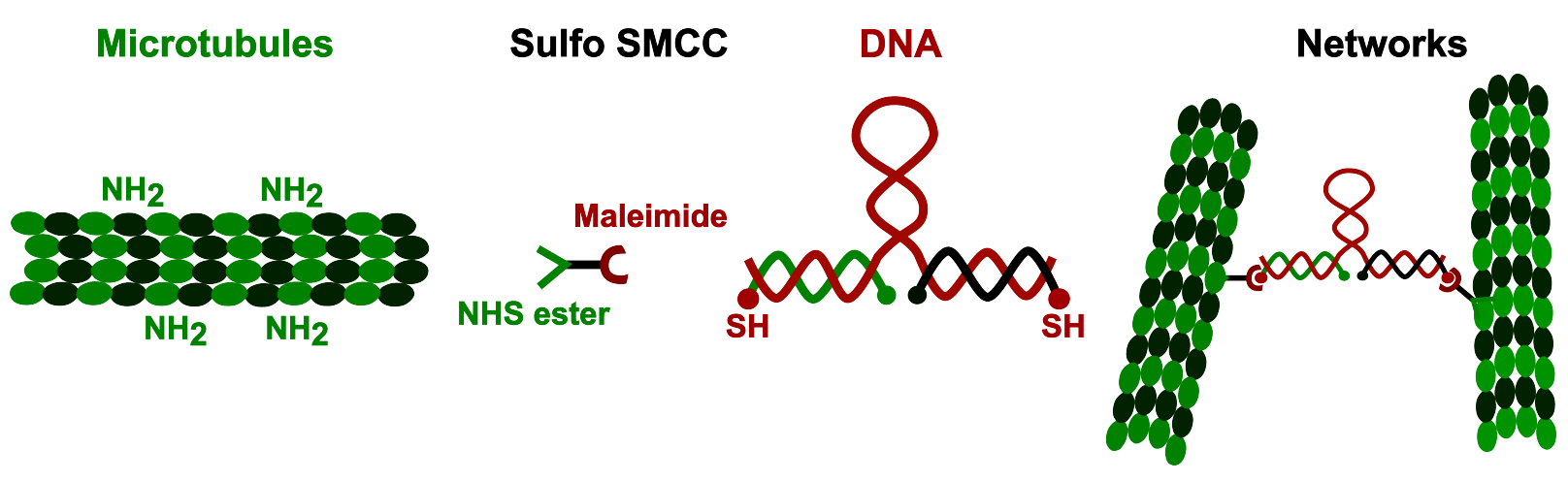

Figure 3.5: Schematic of the crosslinking protocol. NHS ester of crosslinker sulfo-SMCC is reacted with the primary amines present on MTs. DNA with thiol modified ends is added to the mixture and binds to the maleimide end of crosslinker.

A schematic of the crosslinking protocol is shown in Fig. 3.5. A detailed protocol of MT polymerization and DNA preparation is given in Chapter 6. Briefly, MTs were polymerized from a final tubulin concentration of $20 \mu \mathrm{M}$ (referred to as $20 \mu \mathrm{M}$ MTs) and incubated with 250 $\mu \mathrm{M}$ sulfo-SMCC. Thiol modified DNA strands (50 $\mathrm{nM})$ were reduced and added to the MTs and sulfo-SMCC mixture, after removal of the reducing agent, TCEP. The mixture was loaded on rheometer base plate and measured after $1 \mathrm{hr}$ of incubation to allow network formation. 

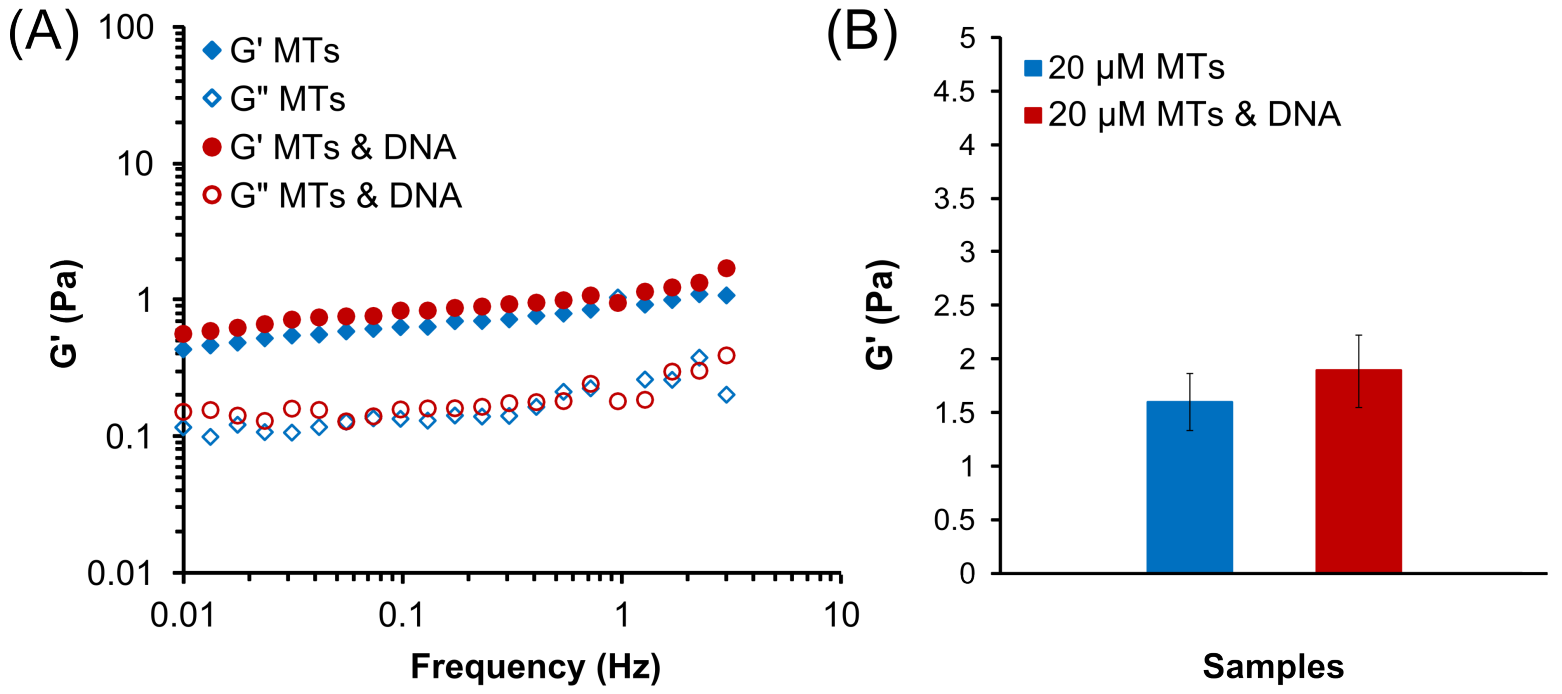

Figure 3.6: Linear viscoelastic measurements of MT networks. (A) Frequency sweep of MTs and MTs crosslinked with DNA shows comparable $G^{\prime}$ and $G^{\prime \prime}$ values for both samples. (B) Linear elastic modulus values from many measurements show that crosslinking MTs with DNA does not significantly change the average $G^{\prime}$ of MTs. Error bars show standard error of mean from 8 and 10 measurements of non-crosslinked and crosslinked MTs, respectively.

\subsubsection{Linear rheology of crosslinked networks}

Comparative frequency sweeps of MTs crosslinked with DNA and non-crosslinked MTs at a small strain of 0.01 are shown in Fig. 3.6A. MTs, with and without crosslinking, show a weak frequency dependence, typically observed for biopolymers. ${ }^{35}$ Also, $G^{\prime}$ dominates over $G^{\prime \prime}$ for both samples, which shows that the samples are more elastic than viscous over the frequency range. Interestingly, $G^{\prime}$ of MTs after crosslinking with DNA remains comparable to $G^{\prime}$ of noncrosslinked MTs. Fig. 3.6B shows average $G^{\prime}$ values at $1 \mathrm{~Hz}$ from multiple measurements of MTs and MTs crosslinked with DNA. The average $G^{\prime}$ values for both, crosslinked and noncrosslinked MTs, lie between 1-2 $\mathrm{Pa}$ and do not differ significantly from each other. Error bars show standard error of mean from 8 and 10 measurements of non-crosslinked and crosslinked MTs, respectively.

\subsubsection{Nonlinearity tests}

The mechanical behavior of networks of stiff rods and flexible crosslinkers is expected to be highly nonlinear from theory and simulations. ${ }^{[32}$ The nonlinear response of networks of MTs crosslinked with DNA can be elucidated experimentally from differential measurements. We tested networks with varying parameters such as concentration of DNA, length of DNA, concentration of MTs and length of MTs. Representative graphs for each condition are shown in Fig. 3.7. Typically, 
we observe that $K^{\prime}$ is constant over a stress range in the linear regime. The differential modulus increases nonlinearly beyond a critical stress and scales as $K^{\prime} \sim \sigma$ until the network ruptures. Note that scaling behavior here is only an approximation, as we have very few data points in nonlinear regime before the sample yields. Thus, we can only characterize true scaling and compare to simulations if we can measure network response over decades of prestress.

A plot of differential modulus over stress of MTs crosslinked with different concentrations of DNA is shown in Fig. 3.7A. Surprisingly, we see that characteristic network response looks similar for weakly, strongly and even non-crosslinked MTs. We also tested networks of MTs crosslinked with a range of DNA lengths from $25 \mathrm{~nm}$ to $1.32 \mu \mathrm{m}$ (see Fig. 3.7B). Long DNA fragments of lengths $1 \mathrm{~kb}$ and $4 \mathrm{~kb}$ were obtained from PCR amplification of plasmid UNC 104. However, we could not elicit any differences in rheological properties for networks of MTs crosslinked with DNA of $L_{\max }=25 \mathrm{~nm}, 330 \mathrm{~nm}$ and $1.32 \mu \mathrm{m}$ from non-crosslinked MTs. We then tested a higher concentration of MTs to reduce the mesh size of networks and thereby increase overlap between filaments for better crosslinking. Fig. 3.7. C shows that the nonlinear behavior of crosslinked MTs resembled its non-crosslinked counterpart even after increasing the concentration of MTs to $40 \mu \mathrm{M}$. Short MTs were obtained by polymerizing tubulin in presence of GMPCPP, instead of GTP. Differential measurements of $20 \mu \mathrm{M}$ short MTs, crosslinked with DNA are shown along with control of non-crosslinked MTs in Fig. 3.7D. The plot shows that shortening MTs does not improve the crosslinking, as the response for crosslinked MTs still follows the non-crosslinked ones. We do not see a nonlinear regime in measurement of short MTs or crosslinked MT networks. However, it must be mentioned that nonlinear regime in the representative plots of long MTs is not always reproducible. The cause of this irreproducibility is unclear. Thus, we cannot comment yet on the nature of interactions between MTs responsible for the nonlinear mechanics or the parameters which they depend on.

Since the nonlinear behavior is independent of crosslinking details, it seems that the observed nonlinear response is majorly contributed by MTs. This is contradictory to our expectations from theory and simulations, which predict that network response at higher stresses should be dominated by DNA. ${ }^{32}$ The above conundrum can be explained by two possible scenarios. In first case, we presumably have networks of MTs crosslinked with DNA but are limited by slippage of networks from rheometer plates prior to actual network rupture. This means that we measure a transition regime at low and intermediate stresses but are unable to capture the actual nonlinear regime with maximum stretching of DNA crosslinks. Another possibility is that the crosslinking procedure does not work as we expect, thus resulting in a weak or no crosslinking at all.

It is difficult to circumvent slippage of sample from the rheometer plates due to limited possibilities of modifications in plate materials. We thoroughly tried troubleshooting for the latter case, testing many variations for optimization of crosslinking protocol. We tested reversing the binding protocol by reacting sulfo-SMCC with DNA first and removal of any excess crosslinker before adding to MTs. An important change in strategy was attaching one strand of DNA to one population of MTs and complementary strand to another population and mixing both populations to hybridize DNA in situ. This measure is useful to avoid looping and attaching of DNA to same MT and thereby enhances crosslinking. However, none of the above mentioned experiments showed any differences between rheology of crosslinked and non-crosslinked MTs.

We also imaged networks with a TCS SP5 X confocal microscope (Leica Microsystems, 

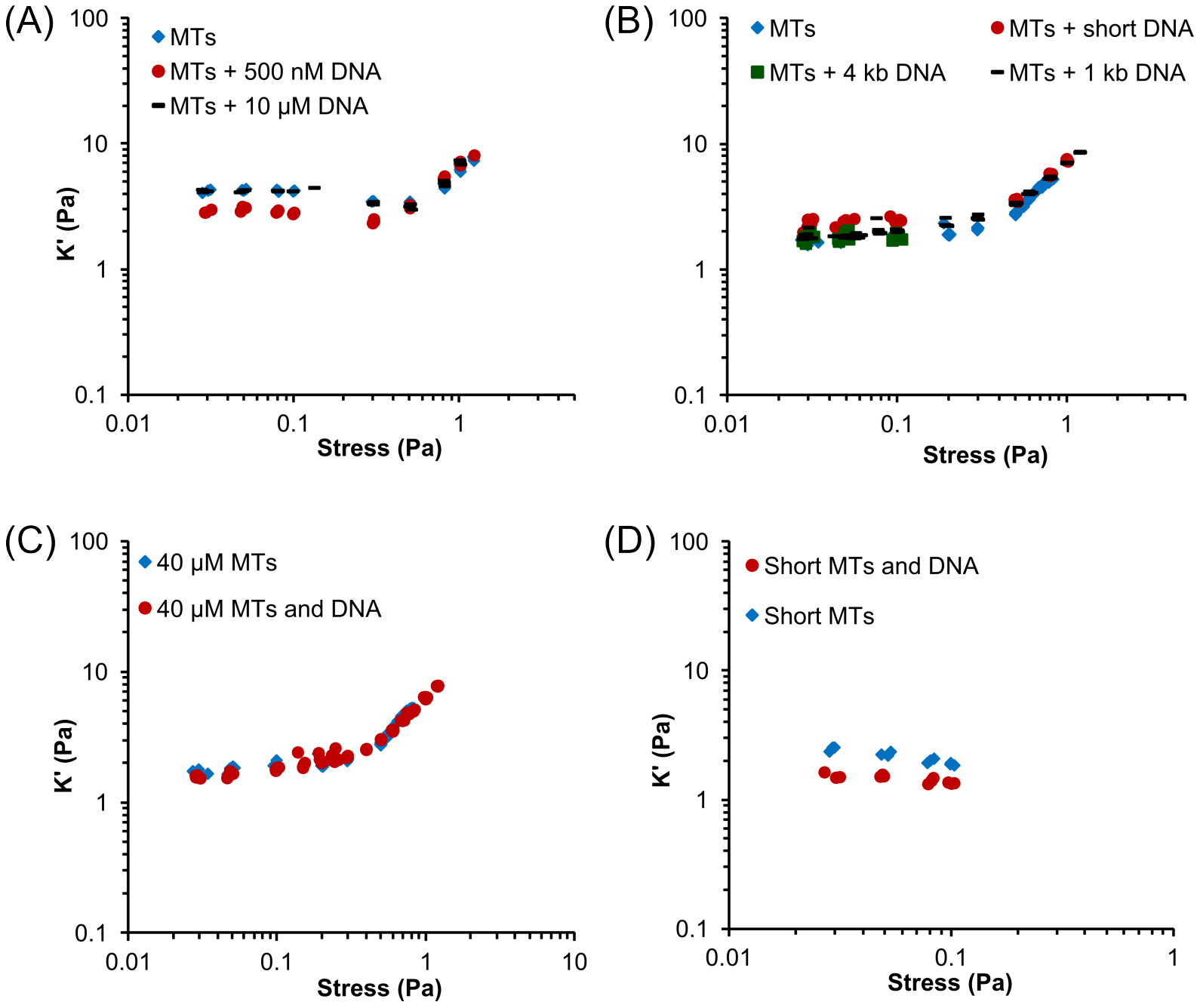

Figure 3.7: Differential measurements of MT networks. Networks of MTs crosslinked with DNA for different parameters. (A) $20 \mu \mathrm{M}$ MTs were crosslinked with different DNA concentrations of $500 \mathrm{nM}$ and $10 \mu \mathrm{M}$. (B) Networks of $20 \mu \mathrm{M}$ MTs crosslinked with DNA lengths ranging from very short DNA of approximately $25 \mathrm{~nm}$ to long DNA of $1 \mathrm{~kb}$ and $4 \mathrm{~kb}$, corresponding to lengths of $330 \mathrm{~nm}$ and $1.32 \mu \mathrm{m}$, respectively. (C) Higher concentration of $40 \mu \mathrm{M} \mathrm{MTs}$, with and without crosslinking. (D) $20 \mu \mathrm{M}$ short MTs, polymerized in presence of GMPCPP, with and without crosslinking. Nonlinear response of crosslinked MTs in all cases is very similar to MTs without DNA crosslinking. 

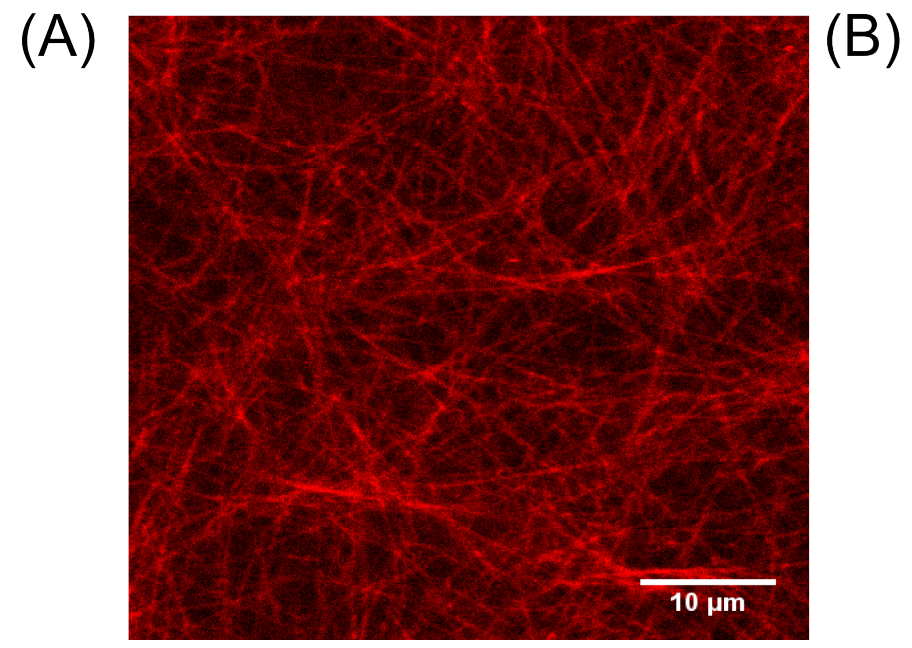

Figure 3.8: Confocal images of networks. (A) MTs and (B) MTs crosslinked with DNA. The network microstructure does not show obvious differences between crosslinked and noncrosslinked MTs.

Mannheim, Germany) to visualize and identify any differences in the network microstructure of crosslinked MTs. Images of high concentration of MTs $(10 \mu \mathrm{M})$ showed many overlaps and entanglements, as shown in Fig. 3.8A. Thus, the non-crosslinked MTs are morphologically very similar to MTs crosslinked with DNA, which are shown in Fig. 3.8B. Therefore, we could not obtain conclusive evidence of crosslinking of MTs from imaging or rheology. Troubleshooting for crosslinking of MTs led to some interesting observations, explained in details in the following sections.

\subsection{Troubleshooting MT crosslinking}

An important check in troubleshooting crosslinking experiments was to verify the attachment chemistry of sulfo-SMCC to MTs and DNA. The binding of maleimide group of sulfo-SMCC to thiols of DNA was confirmed through the DNA immobilization experiments on coated coverslips, as described in Section 2.6. The attachment of NHS ester group to the primary amines of MTs was tested by using a Alexa $488 \mathrm{NHS}$ ester dye. The dye was incubated with MTs in two different buffer conditions; HEPES buffer ( $\mathrm{pH}$ 8) and BRB $80(\mathrm{pH} 6.8)$. While HEPES provides the basic $\mathrm{pH}$ conditions optimum for NHS ester chemistry, BRB 80 is an ideal buffer system for microtubules. Fig. 3.9 shows that the NHS ester dye labeled MTs in both the buffer conditions, thus confirming the attachment chemistry. 
(A)

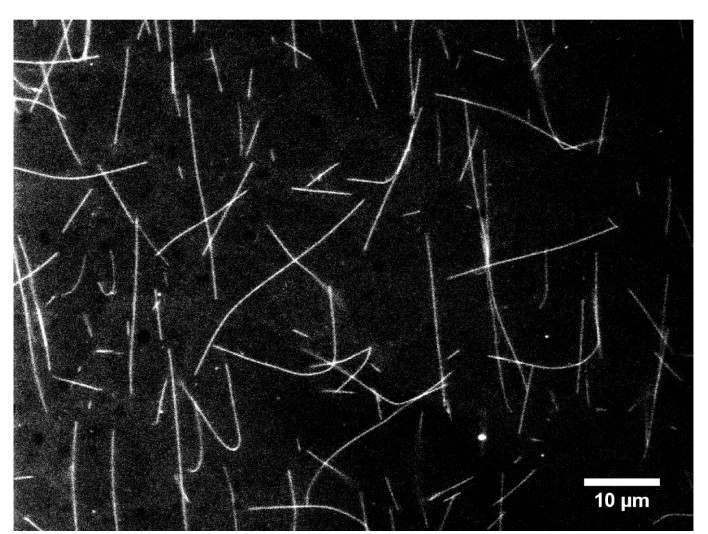

(B)

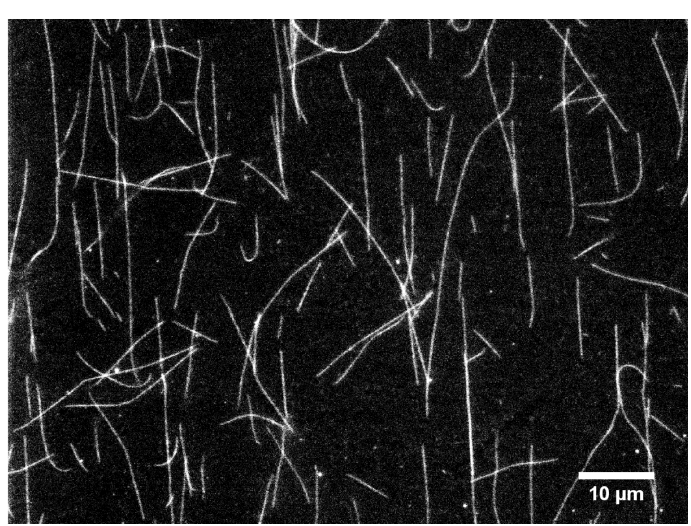

Figure 3.9: NHS ester dye labeling primary amines of MTs. MTs were incubated with Alexa 488 NHS ester dye for $1 \mathrm{hr}$ in (A) HEPES ( $\mathrm{pH}$ 8) or (B) BRB 80 buffer ( $\mathrm{pH} \mathrm{6.8).}$

\subsection{Sulfo-SMCC prevents annealing of MTs}

Microscopy showed some interesting effects on length distribution of MTs on addition of crosslinker, sulfo-SMCC. As sulfo-SMCC consists of a N-hydroxysuccinimide (NHS) ester and a maleimide group, control experiments were done by testing effects of maleimide and NHS ester groups, individually, on MTs. MTs were incubated with $250 \mu \mathrm{M}$ each of sulfo-SMCC, Alexa 488 malemide dye (Life Technologies GmbH, Darmstadt, Germany) and Alexa 488 NHS ester dye (Life Technologies GmbH, Darmstadt, Germany) and imaged after 24 hrs. Negative control sample consisted of MTs without any chemical treatment. Details of image processing and analysis of length distributions are given in Chapter 6.

Fig. 3.10 shows length distribution calculated from images of MTs after 24 hrs incubation for all treatment conditions. Solid lines show exponential fits for length distribution of MTs for all cases, as previously reported for biopolymers. ${ }^{35137}$ We observed that MTs treated with sulfo-SMCC are drastically shorter in comparison to untreated MTs with almost a magnitude of difference in their mean lengths $(2 \mu \mathrm{m}$ and $17 \mu \mathrm{m}$, respectively). Curiously, maleimide dye treatment showed only a two-fold difference in mean length $(9.2 \mu \mathrm{m})$ from untreated MTs. It is unclear why the effect of sulfo-SMCC is much more pronounced than maleimide dye, although they bear the identical reactive group. We, therefore, tested a control sample to check for an effect of NHS ester group from sulfo-SMCC on MT lengths. The length distribution after NHS ester treatment of MTs overnight, however, was very similar to untreated MTs, thereby negating the possibility of its effect on MTs.

It is clear that $24 \mathrm{hrs}$ treatment with sulfo-SMCC results in much shorter MTs than untreated ones. To further understand the sulfo-SMCC induced MT 'shortening' effect, we compared length distributions of chemically treated and untreated MTs after short (2 hrs) and long (24 hrs) incubation times. Fig. 3.11 shows the graphs and corresponding representative images of short (S) and long (L) time incubations for each condition. MTs without treatment elongated approximately three times their original mean length after overnight incubation due to end to end annealing, as shown in Fig. 3.11 A. Addition of a maleimide dye also showed a similar trend, 


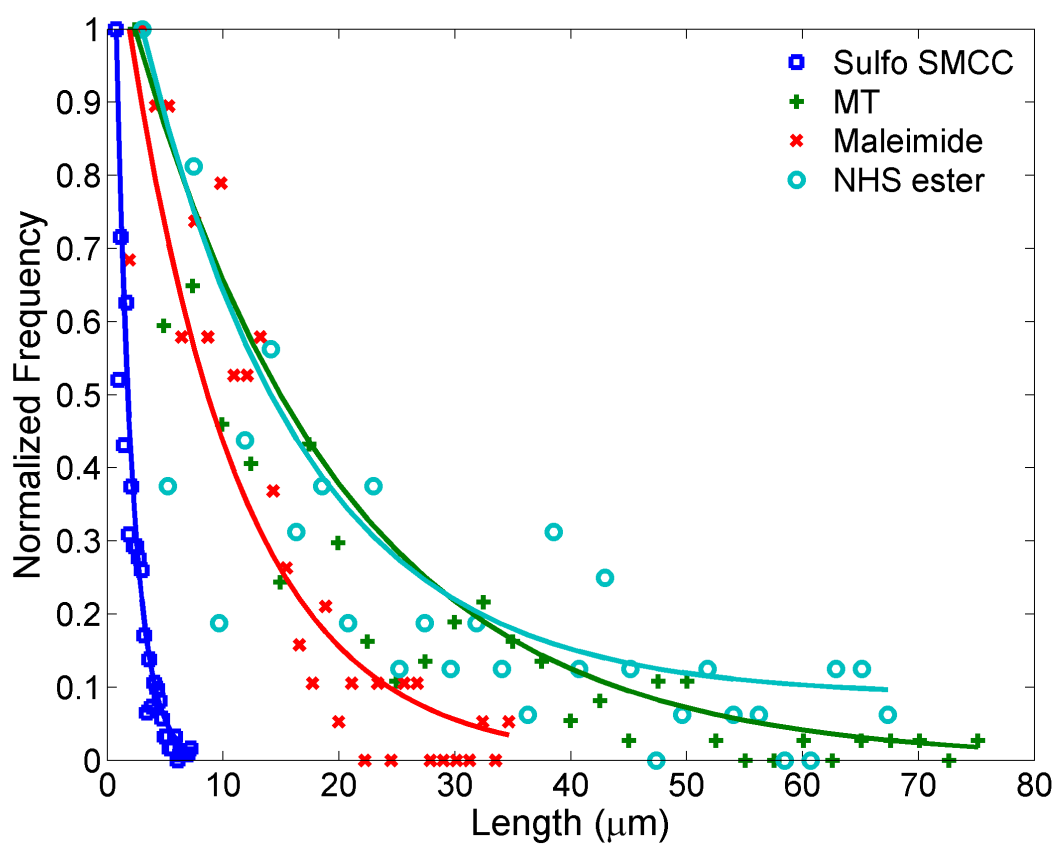

Figure 3.10: Length distribution of treated and untreated MTs after long incubation. Length distribution of $20 \mu \mathrm{M}$ untreated MTs, MTs treated with $250 \mu \mathrm{M}$ sulfo-SMCC, $250 \mu \mathrm{M}$ maleimide dye and $250 \mu \mathrm{M}$ NHS ester dye is shown. Length of MTs, in all cases, is distributed exponentially; fits shown as solid lines. Treatment with sulfo-SMCC resulted in drastically shorter MTs in comparison to untreated MTs (mean length was $2 \mu \mathrm{m}$ and $17 \mu \mathrm{m}$, respectively). Maleimide dye treated MTs, however, showed a less drastic effect with an intermediate mean length of $9.2 \mu \mathrm{m}$. Addition of NHS ester dye to MTs as control did not affect the length of MTs.

as shown in Fig. 3.11B. This implies that the presence of a maleimide dye does not drastically alter the annealing process. On the contrary, MTs treated with sulfo-SMCC maintained a temporally constant mean length of approximately $2 \mu \mathrm{m}$, as shown in Fig. 3.11C. This result suggests that sulfo-SMCC induced 'shortening' of MTs is, in fact, prevention of end to end annealing.

Many previous studies have identified sulfhydryl $(\mathrm{SH})$ groups from cysteines of tubulin to be essential for MT polymerization; first demonstrated by blocking them with chemicals such as PCMPS, NEM and DTNB. ${ }^{38 / 39]}$ MT assembly was shown to be affected in vitro as well as in vivo, in these experiments. Experiments with isothiocyanates have also shown to induce mitotic arrest and apoptosis in cells by covalent modification of cysteines in tubulin. ${ }^{40}$ An interesting result showed preferential inhibition of minus end assembly by mixing NEM-tubulin with regular tubulin during MT polymerization. ${ }^{33 / 41}$ Although the exact mechanism is not known, it is postulated that NEM binding to tubulin $\mathrm{Cys}^{239}$ may be responsible for the minus end capping mechanism. We speculate from our results that vital $\mathrm{SH}$ groups on cysteine residues that are essential in MT polymerization are also important for annealing of MTs. 
(A)

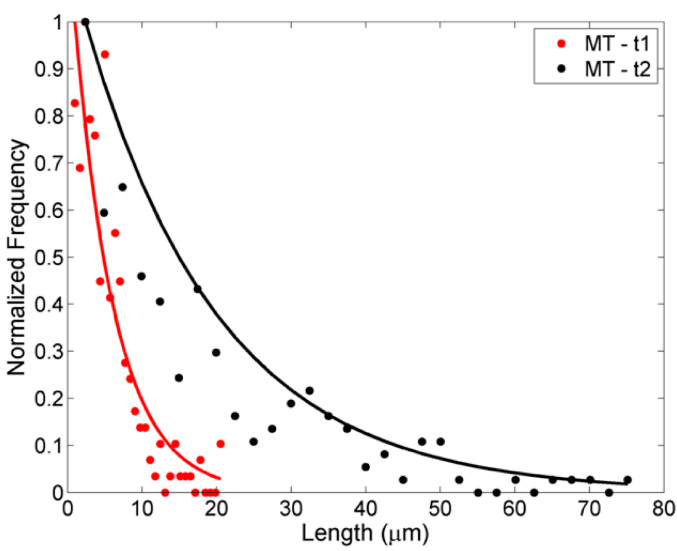

(B)

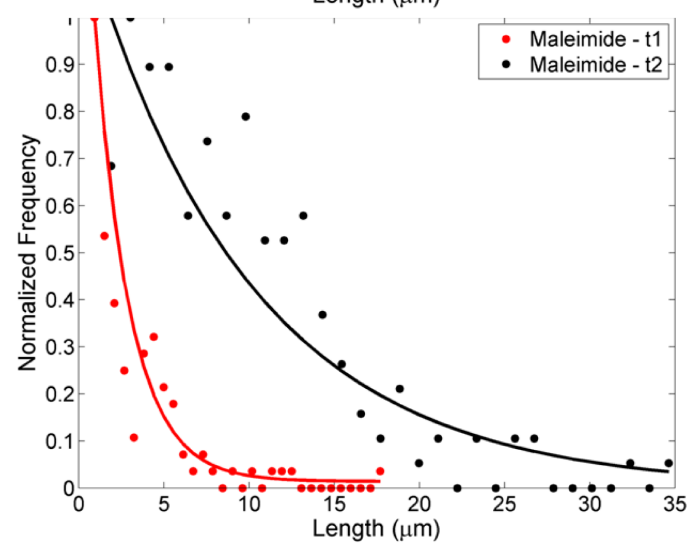

(C)

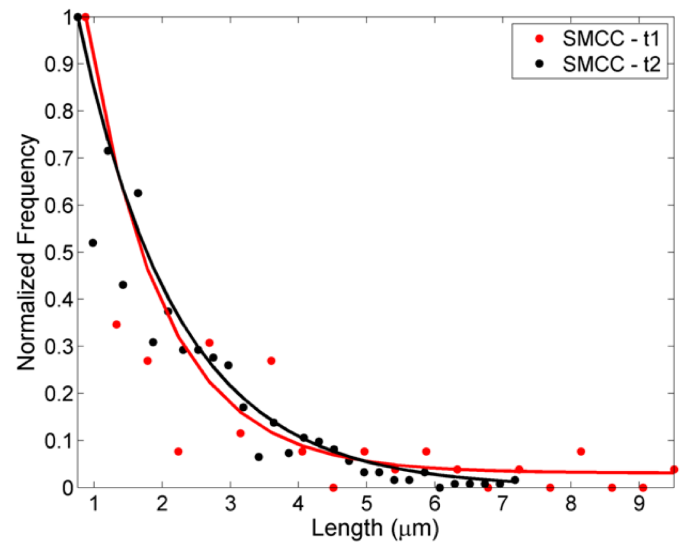

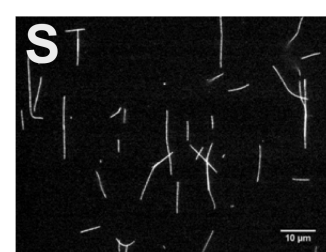
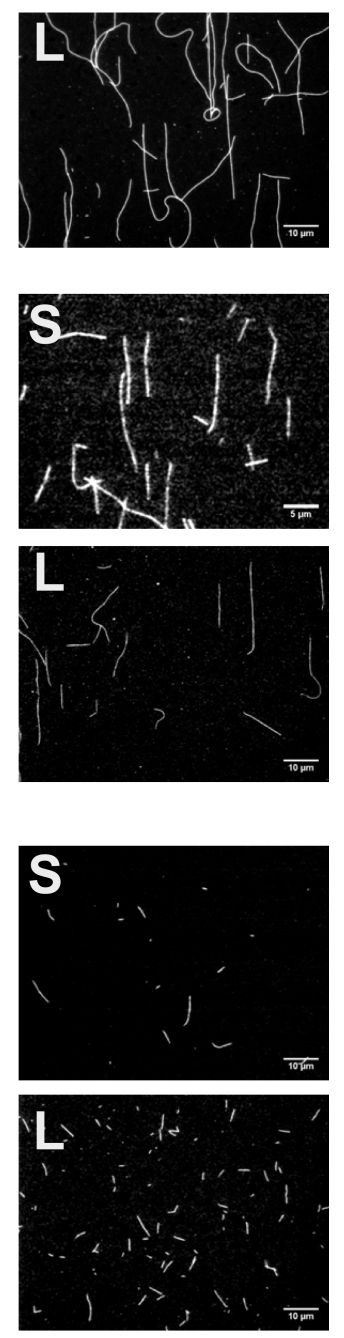

Figure 3.11: Length distributions of treated and untreated MTs after 2 hrs and 24 hrs incubation times. Length distributions of $20 \mu \mathrm{M}$ untreated MTs and MTs treated with 250 $\mu \mathrm{M}$ maleimide and $250 \mu \mathrm{M}$ sulfo-SMCC after 2 hrs (short) and 24 hrs (long) incubation times are shown in (A), $(B)$ and $(C)$, respectively. Red and black circles represent data from short and long time incubation, respectively. Solid lines show exponential fits of data. Corresponding images are also shown next to the graphs, where $\mathrm{S}$ and $\mathrm{L}$ denote short and long incubation times, respectively (scale bar $=10 \mu \mathrm{m}$ ). We see that mean lengths of both, untreated and maleimide treated MTs, increased approximately 3-fold from short to long time incubation due to temporal end of end annealing of MTs. Sulfo-SMCC treatment, however, inhibited annealing of MTs and maintained a mean length of approximately $2 \mu \mathrm{m}$. 
Interestingly, all the previous studies demonstrated deleterious effects of chemicals blocking the $\mathrm{SH}$ groups on the polymerization of MTs. Polymerized and stabilized MTs have been assumed to be insusceptible to these chemicals. It is therefore, surprising and also important to know that a commonly used crosslinker has a huge impact on MTs structure and dynamics, even after stabilization. Although, the exact mechanism of how sulfo-SMCC prevents annealing in MTs is not clear so far, MTs are clearly susceptible to cysteine binding chemicals and special care must be taken while dealing with the same.

\subsection{Crosslinking of MTs with biotin and neutravidin}

The last two sections showed that rheological response of MTs crosslinked via sulfo-SMCC is indistinguishable from non-crosslinked MTs. Also, sulfo-SMCC prevents annealing of MTs, which results in different length distributions of crosslinked and non-crosslinked MTs over long time scale experiments ( $24 \mathrm{hrs}$ ). In order to find out if the weak rheological response is an effect of poor crosslinking with sulfo-SMCC or an inherent property of MTs themselves, we tested an alternative crosslinking strategy.

Biotin-neutravidin linkage has been commonly used for crosslinking biopolymers, including microtubules. ${ }^{35 / 36 / 42 / 44}$ Neutravidin has four binding sites for biotin and attaches with a high specificity and affinity of $K_{d}=10^{-15} \mathrm{M}$. We crosslinked biotinylated MTs with varying concentrations of neutravidin and measured linear moduli over a frequency range, as shown in Fig. 3.12A. MTs crosslinked with different concentrations of neutravidin, surprisingly, do not differ in their linear moduli from non-crosslinked MTs.

We measured networks of biotinylated actin with similar neutravidin concentrations as those used for MT crosslinking, as a positive control. Fig. 3.12B shows a plot of differential modulus over prestress of crosslinked and non-crosslinked actin and MTs. The ratio of crosslinking for actin networks shown in plot was $R_{N}: R_{B}=1$. Crosslinked actin networks show more than a magnitude higher $G^{\prime}$ values in linear range in comparison to non-crosslinked actin. The observed behavior of actin networks compares well with similar studies reported previously. 45 The crosslinked networks also display a short nonlinear regime and yield at approximately 10 -fold larger stress than entangled actin.

Fig. 3.13 shows confocal images of same concentrations of entangled actin and networks of neutravidin crosslinked biotinylated actin. Images of actin crosslinked with neutravidin show a clearly different network structure with clusters of actin filaments and a larger mesh size, in comparison to entangled actin. Our results show that crosslinked actin networks show drastically different rheological properties from entangled actin. In contrast, mechanical properties of crosslinked MT networks closely resemble those of non-crosslinked MTs, irrespective of the method of crosslinking. This is intriguing, as bulk rheology on microtubule networks of comparable concentrations has demonstrated up to 6-fold difference between $G^{\prime}$ values of crosslinked and non-crosslinked networks. ${ }^{35}$ We speculate that the discrepancies arise possibly due to different experimental parameters. Mechanical properties of such composite networks are known to be susceptible to length of MTs, for instance. ${ }^{32}$ Long MTs are more likely to be geometri- 

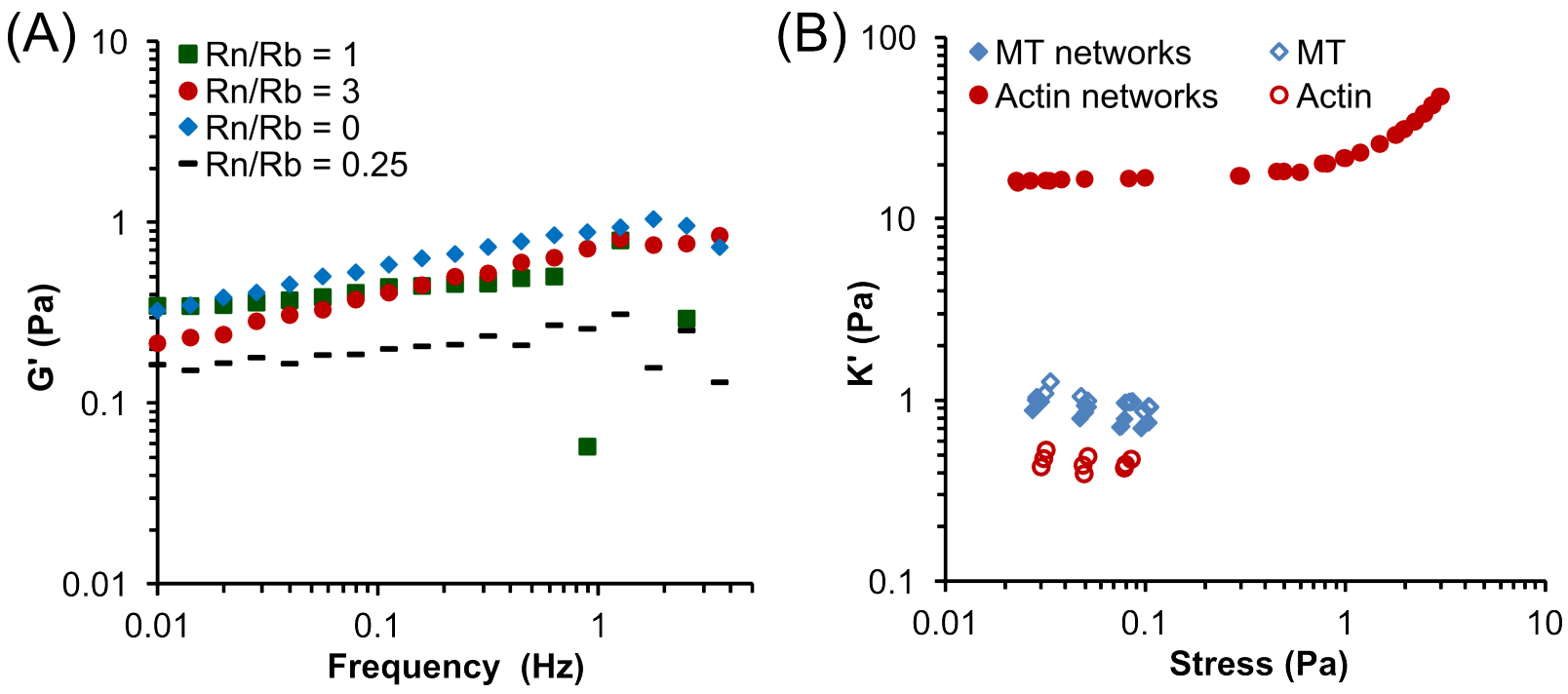

Figure 3.12: Biotin-neutravidin crosslinked networks. (A) Frequency sweeps of biotinylated MTs crosslinked with varying neutravidin concentrations do not show drastic differences in linear modulus values. (B) Comparison of differential measurements of biotinylated actin crosslinked with neutravidin to crosslinked MTs. Ratio of crosslinking for networks was $R_{N}: R_{B}=1$. Actin networks show more than a magnitude higher $G^{\prime}$ values after crosslinking with neutravidin. Networks of crosslinked actin are nonlinear and the yield stress is much larger than non-crosslinked actin. Mechanical response of MTs, on the contrary, does not change after crosslinking.

cally frustrated with a few crosslinks than short MTs. It is known that GTP polymerized MTs are longer than MTs polymerized in presence of GMPCPP. Therefore, mechanical properties of networks of long MTs in our experiments may not match the short MT network studies. A detailed study of characterization of network mechanics of varying MT lengths is required to confirm our speculation.

We conclude from our observations with biotin-neutravidin networks that our problems with crosslinking of MTs are not necessarily dependent on the crosslinking strategy. In general, it seems that mechanical response of MT networks is complicated and difficult to interpret from rheometry. Actin networks, on the contrary, show drastic differences in their mechanical properties on crosslinking. Furthermore, actin can be treated as stiff rods, depending on the degree of crosslinking. $\stackrel{46}{ }$ We can therefore generate the desired composite networks experimentally by crosslinking actin, instead of microtubules, with DNA. The next section describes actin crosslinking with DNA using Halotag fusion proteins.

\subsection{Actin crosslinking with Halotag}

As described in Section 3.4, actin networks have been commonly crosslinked using actin binding proteins (ABP). Designing a crosslinking strategy with $\mathrm{ABPs}$ has two important advantages. 

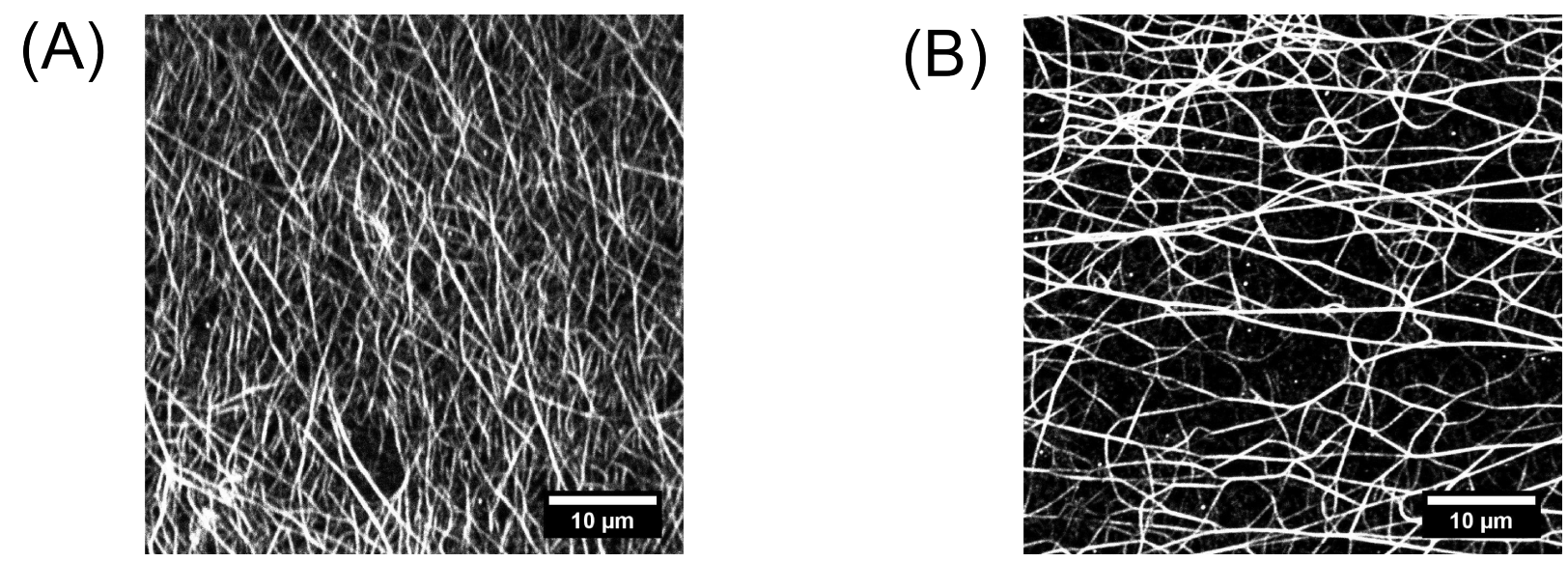

Figure 3.13: Images of entangled and crosslinked actin. Confocal images of same concentration of $(A)$ entangled actin and (B) biotinylated actin crosslinked with neutravidin. The network architecture of crosslinked actin shows cluster of filaments and a relatively larger mesh size, while entangled actin has a homogeneous, fine meshwork.

First of all, networks of actin crosslinked with ABPs are biologically more relevant than an artificial system such as biotin-neutravidin. Secondly, ABP fusion proteins can be easily generated to attach another component, in our case DNA, specifically to actin and avoid any crossreactions.

We developed a strategy utilizing a fusion protein of Lifeact with Halotag. Lifeact is a 17 amino acid long part of an ABP, $\alpha$-actinin, that is routinely used for labeling actin in live cells. $\frac{47}{4}$ Halotag binding strategy is based on the formation of a covalent bond between a protein fusion tag and a Haloligand (PROMEGA GMBH, Mannheim, Germany). Detailed preparation of the fusion protein is given in Chapter 6 . In brief, the Lifeact-Halotag fusion protein with a His tag is expressed in E. coli and purified using a Ni-NTA column. In our experiments, the Haloligand is functionalized with iodoacetamide, which reacts with thiol ends of the DNA sensor. A schematic of the binding strategy is shown in Fig. 3.14. The purified fusion protein was incubated with actin filaments in vitro, in a molar ratio of $10: 1$, to test for binding specificity. Confocal images of actin, with and without protein, are shown in Fig. 3.15. The images showed that the protein bound to actin but also resulted in aggregation of actin filaments. A gel confirming binding of Lifeact-Halotag protein is shown in Fig. 3.17A.

One of the reasons for protein aggregation could be chelation of nickel ions during purification of His tagged protein. We added different chemicals to the elution buffer, such as $1 \mathrm{mM}$ EDTA, $0.1 \%$ Triton-X 100 and $0.1 \%$ Tween 20 , to address this possibility. However, the purified protein in all cases still resulted in protein aggregation, as shown in Fig. 3.17B. Over-expression of proteins can also result in a misfolded structure, leading to aggregation. We down-regulated protein expression by inducing stress in E.coli by addition of $5 \%$ ethanol and/or lowering the incubation temperature to $25^{\circ} \mathrm{C}$. The native PAGE gel, shown in Fig. 3.17C showed reduced aggregation of protein on combining addition of $5 \%$ ethanol and incubation at $25^{\circ} \mathrm{C}$.

Thus, we now have a fusion protein that can be used for crosslinking actin with DNA. The next steps for in vitro experiments would be characterization of network mechanics with the rheometer. Also, we aim to continue with the force sensing experiments to demonstrate working 


\section{Actin}

\section{DNA sensor}

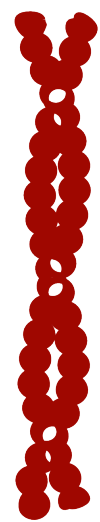

\section{Lifeact-Halotag}
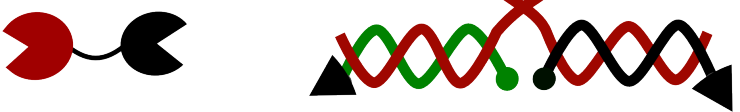

Haloligand
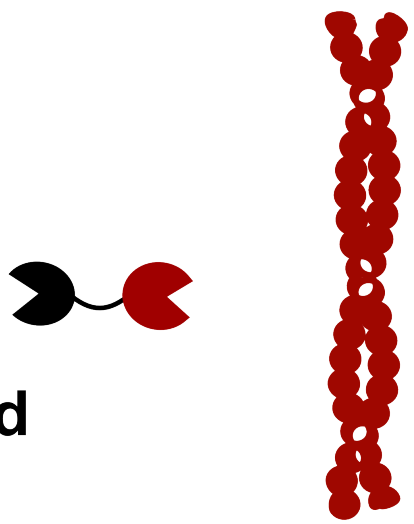

Figure 3.14: Schematic of crosslinking actin using Halotag binding strategy. Haloligand, functionalized with iodoacetamide is incubated with thiolated DNA. Lifeact-Halotag fusion protein is expressed in E.coli and purified. The Lifeact part will attach specifically to actin while Halotag forms covalent bonds with Haloligand attached to DNA, thus crosslinking actin.

(A)

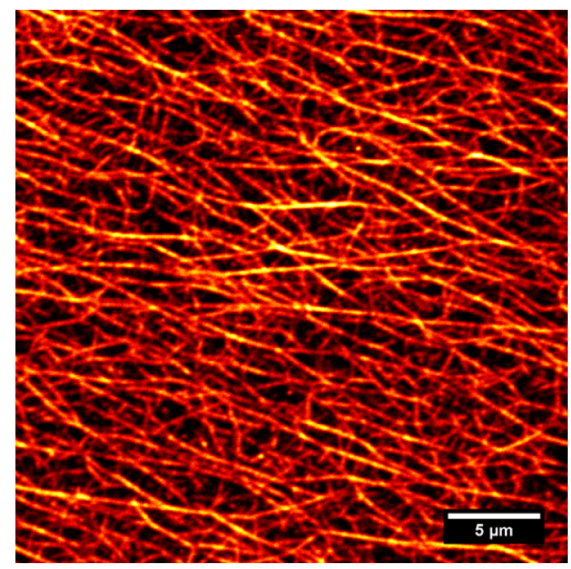

(B)

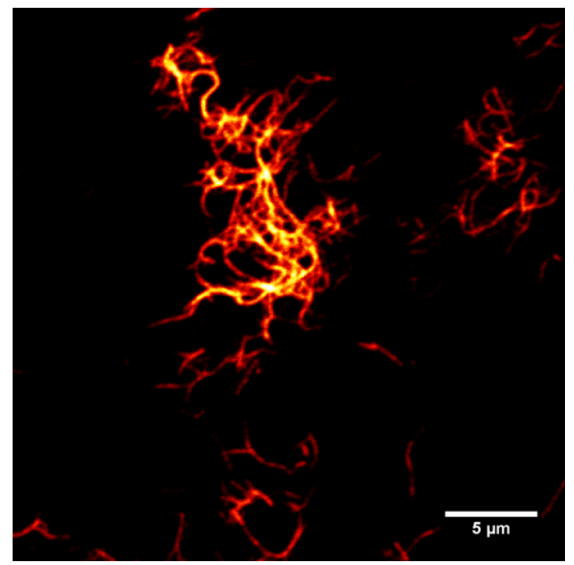

Figure 3.15: Aggregation of actin with Lifeact-Halotag protein. Confocal images of $(A)$ actin and (B) actin with Lifeact-Halotag. Actin forms a homogeneous carpet-like fine mesh, while addition of Lifeact-Halotag results in aggregated bundles of actin.

(A)

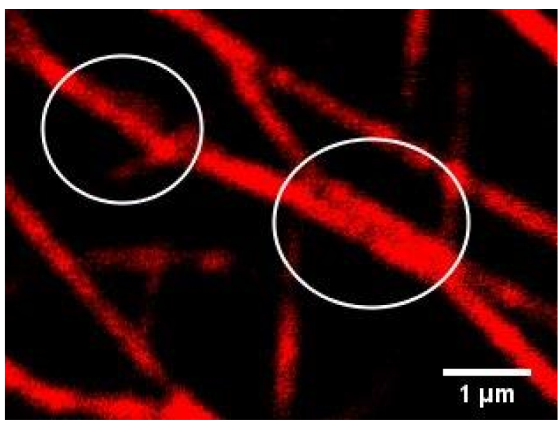

(B)

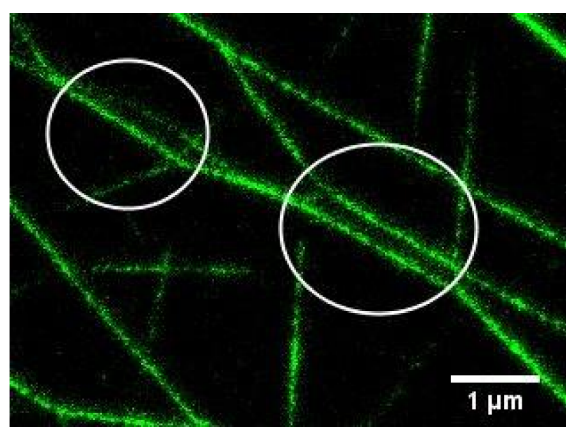

Figure 3.16: Confocal and STED imaging of actin. Actin imaged using (A) confocal and (B) STED microscopy. The branching of filaments (white circles) and general microstructure is resolved much better using STED microscopy. 


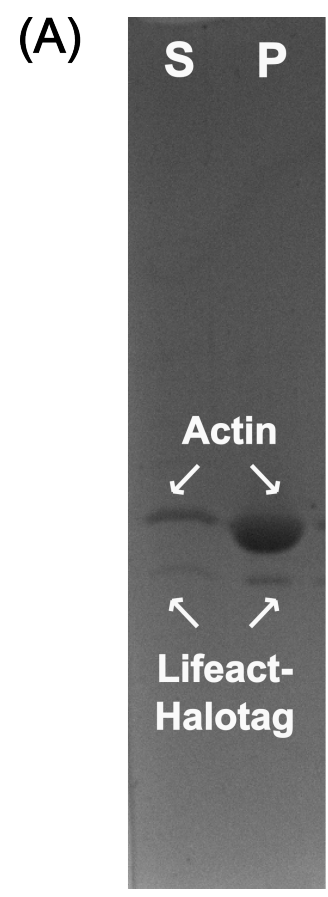

(B)

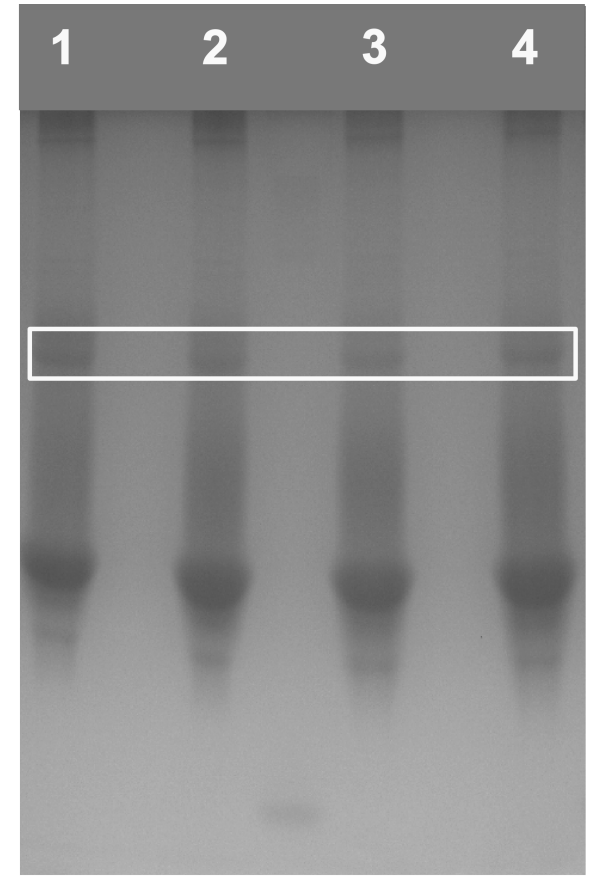

(C)

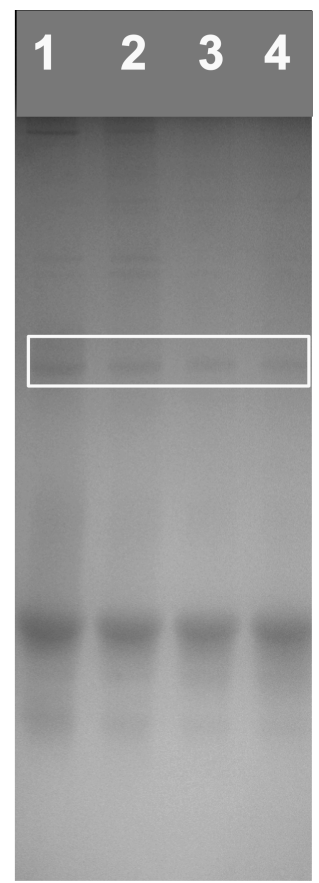

Figure 3.17: Gels showing Lifeact-Halotag purification and binding to actin. (A) Binding assay of the fusion protein Lifeact-Halotag with actin showed that the protein bound to actin and largely co-sedimented with it in the pellet fraction $(P)$. The supernatant (S) consisted of a small amount of both, actin and the protein. (B) Electrophoreses of purified protein fractions of Lifeact-Halotag using different elution conditions. The protein aggregated (white box) under all elution conditions of no addition in elution buffer (lane 1), adding $1 \mathrm{mM}$ EDTA (lane 2), 0.1\% Triton-X 100 (lane 3) and $0.1 \%$ Tween 20 (lane 4). (C) Other ways of avoiding protein aggregation was to reduce protein expression by stressing E.coli with addition of $5 \%$ ethanol (lane 2 ) or lowering incubation temperature to $25^{\circ} \mathrm{C}$ (lane 4). The aggregated protein band, marked by a white box, was especially reduced with a combination of ethanol and lowering temperature (lane 3 ) in comparison to the protein purified using normal protocol (lane 1).

of our DNA force sensors in these artificial networks using optical traps.

Imaging of networks will be done using stimulated emission depletion microscopy (STED). The advantage of STED microscopy is its high resolution to visualize fine details in network micro-structure; especially relevant at high polymer concentrations. ${ }^{48}$ An example of actin sample imaged in confocal and STED microscope is shown for comparison in Fig. 3.16 A, B, respectively. Comparison of areas marked in white circles in the images shows that branching and small clusters of filaments, which are not resolved in confocal images, are clearly visible in the high resolution STED images.

An important advantage of this strategy is that it can also be extended to in vivo experiments. We also briefly tested introduction of our DNA sensor along with in vitro purified fusion protein into cells. These experiments are described and discussed in Chapter 4. 


\subsection{Possible artifacts in rheometry}

The rheometer is a handy tool to measure the viscoelastic properties of bulk solutions. However, it is prone to certain artifacts that are important to be aware of while working with it. Some of the key points of discrepancies that we realized during our experiments are described below.

\subsubsection{Volume matters}

The motivation for the experiments explained below arose from a paper showing jamming transition based on volume fraction of an emulsion of castor oil in water. ${ }^{49}$ During our rheology experiments with microtubules, we came across an interesting observation. Shear rate sweeps on varying concentrations of MT solutions interestingly showed a behavior reminiscent of jamming transition in emulsions. The stress characterized as a function of shear rate is shown in Fig. 3.18A. The behavior at different concentrations of MTs could be categorized into 2 distinct regimes. The flow curves at $c>c_{c r}$ showed a finite yield stress at low shear rates and exhibited shear thinning at high shear rates. At lower concentrations of $c<c_{c r}$, we observed Newtonian fluid-like behavior at low shear rates and shear thinning at high shear rates. The critical concentration, $c_{c r}$, was the transition point between two regimes.

The flow curves could also be scaled onto a master curve by appropriate scaling of the axes with respect to critical concentration. The rescaled flow curves are shown in Fig. 3.18B. Since we correct for viscous response of the buffer, the subcritical branch disappears on rescaling the curves.

This result was extremely surprising and interesting, since the behavior of MTs cannot be described by jamming. The volume fraction is very low at $c_{c r}(0.001)$ in comparison to that required for athermal jamming transition (0.64). 50 However, due to problems in reproducibility of experiments, we investigated deeper into this issue with control experiments without microtubules. We observed with control experiments on PBS buffer that simply changing the volume of buffer in the rheometer resulted in different flow curve regimes. Fig 3.19A shows representative curves from 3 different sample volumes - $70 \mu \mathrm{l}$ (underfilled), $80 \mu \mathrm{l}$ (correctly filled) and $120 \mu \mathrm{l}$ (overfilled). The flow curve of an underfilled buffer volume of $70 \mu \mathrm{l}$ looks similar to the 'critical regime' in MT experiments. Fig. 3.19B shows evident resemblance of flow curve of $70 \mu \mathrm{l}$ of PBS plotted together with $10 \mu \mathrm{M}$ MTs. Thus, we concluded that different regimes observed in flow curves of varying concentration of microtubules are probably artifacts arising from volume changes in solvent.

A recent paper gives a model explaining the cause and effect of volume-based artifacts in rheometer measurements. ${ }^{51}$ In an ideal case, one would not expect torque to be generated between the rheometer plates in a rotationally symmetric geometry. However, asymmetries in contact line at sample edge, depending on wetting conditions and evaporation, result in differential surface tension along sample edge. The torque, arising due to the generated surface tension, can be misinterpreted as inaccurate shear thinning of fluids or give an apparent elastic modulus in oscillatory measurements. 

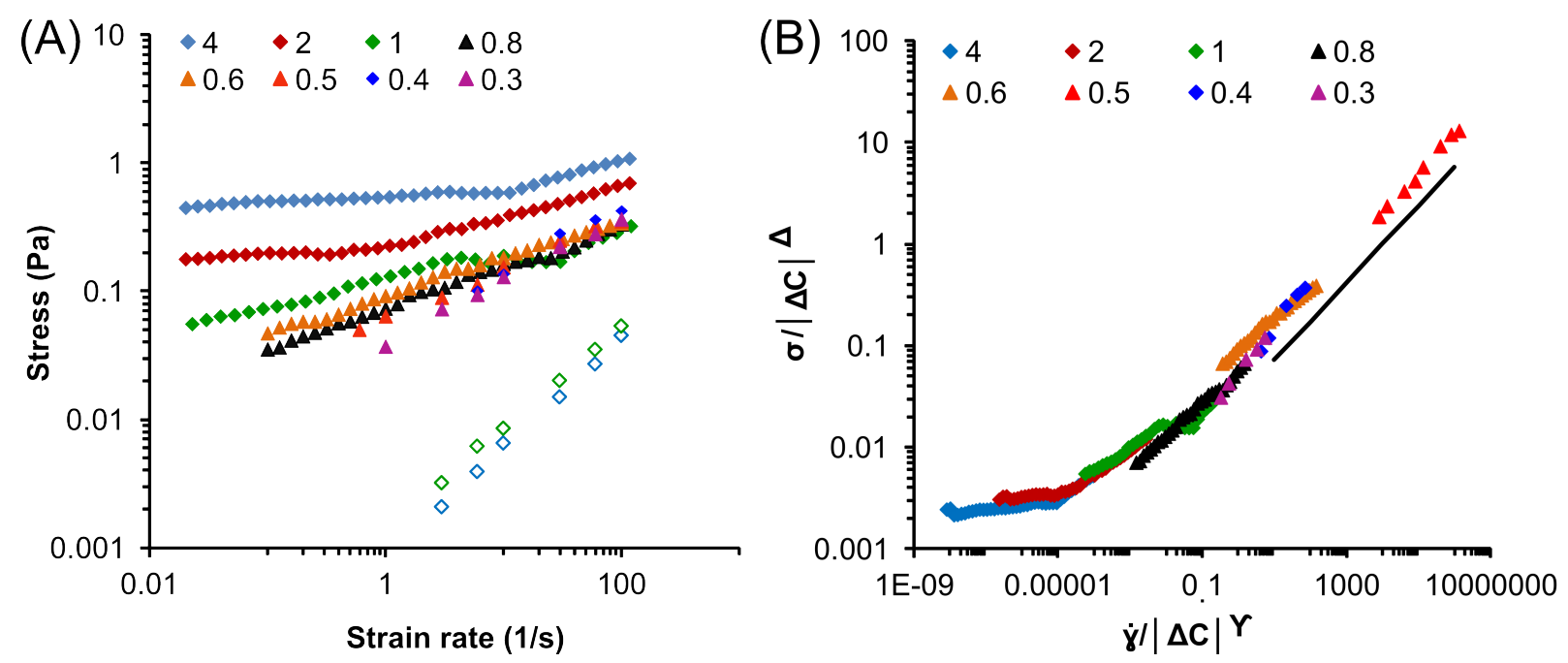

Figure 3.18: Critical gelation of microtubules. (A) Stress as a function of strain rate for different concentrations of microtubules $(\mathrm{mg} / \mathrm{ml})$. MTs showed critical behavior between concentrations of $0.3-0.5 \mathrm{mg} / \mathrm{ml}$ (corresponding to a volume fraction of approximately 0.001). (B) Plot of $\sigma /|\Delta c|^{\Delta}$ vs $\dot{\gamma} /|\Delta c|^{\gamma}$ showing collapse of flow curves of different MT concentrations onto a master curve $\left(c_{c r}=0.5 \mathrm{mg} / \mathrm{ml}\right)$.

Some studies have tried to correct for the volume-based artifacts in cone and plate rheometers. The methods include implementing modification in rheometer design itself or applying corrections based on sample radius monitored throughout the readings. $\frac{52.53}{\text { In }}$ general, the knowledge that changes in volume largely affect low torque measurements is of special importance for soft biopolymer samples (also see Section 3.10.3). It also necessitates choosing time scales of experiments such that they are devoid of these artifacts. For instance, our rheometer does not have an inbuilt solvent trap system to avoid evaporation of sample. We maintain humidity in our experiments by surrounding the sample with wet tissues. This method works for a short time course (few hours) of experiments but one must be aware of the possible issues arising due to evaporation over long time experiments.

\subsubsection{Bottom plate dilemma}

The two common materials used for bottom plate of the rheometer are stainless steel and chromium oxide. While both are chemically inert, the former is more widely used for biopolymer networks while the latter is more useful in temperature sensitive experiments due to better heat conductivity. Our rheometer is equipped with an chromium oxide bottom plate and therefore, most experiments were performed using this plate. However, in order to test whether we find any differences in our measurements based on material of bottom plate, we compared samples on both plates. 

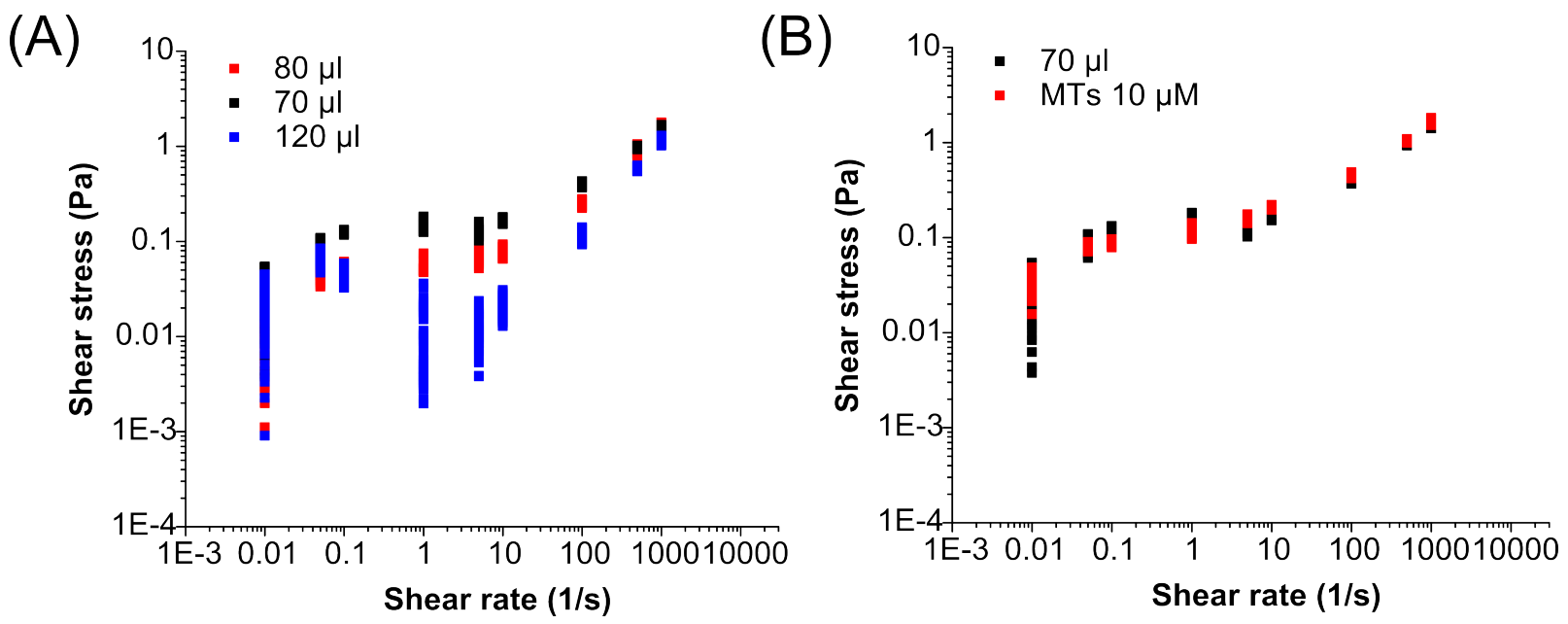

Figure 3.19: Volume based artifacts in the rheometer. (A) $70 \mu \mathrm{l}$ (underfilled), $80 \mu \mathrm{l}$ (correctly filled) and $120 \mu \mathrm{l}$ (overfilled) volume measurements of PBS buffer. Underfilling buffer volume $(70 \mu \mathrm{l})$ resembles the critical regime seen in MT experiments. (B) Flow curves of $70 \mu \mathrm{l}$ of PBS and $10 \mu \mathrm{M}$ MTs. Control sample of buffer show same behavior as critical concentration of MTs.

Fig. 3.20 shows a custom-made stainless steel plate, which could be fitted on top of the existing chromium oxide plate of rheometer. The plate was roughened using sandpaper prior to mounting, to minimize slippage of sample from the plate.

Fig. 3.21 shows frequency sweeps of actin and BRB 80 buffer on an externally attachable stainless plate in comparison to those obtained on a regular chromium oxide bottom plate. The $G^{\prime}$ and $G^{\prime \prime}$ values of actin are higher with the stainless steel plate as compared to chromium oxide plate, as shown in Fig. 3.21A. Comparison of BRB 80 buffer

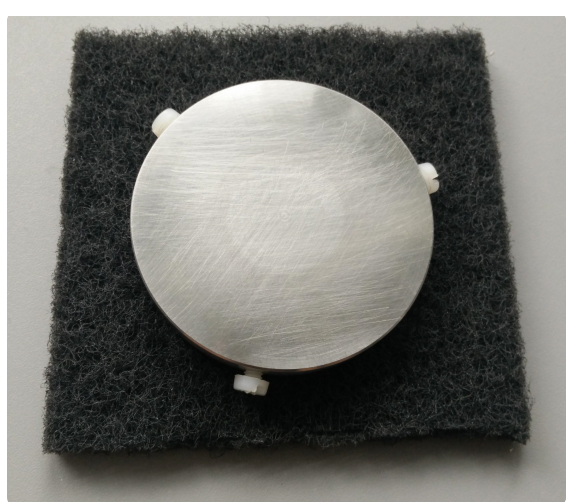

Figure 3.20: Stainless steel bottom plate of rheometer. on both plates also shows slightly higher $G^{\prime}$ values with the stainless plate, as shown in Fig. 3.21B. Similar experiments with polyacrylamide gels (data not shown) also showed consistently higher $G^{\prime}$ values on stainless plate, while those with hyaluronic acid gels (data not shown) showed an opposite trend with lower $G^{\prime}$ on stainless plate. This suggests that shear moduli measured on different plates may be influenced by material dependent differential spreading of the sample.

It is unclear whether the $G^{\prime}$ values obtained from either of the plates are universally real or if suitability of the bottom plate material is sample dependent. It is, however, important to 

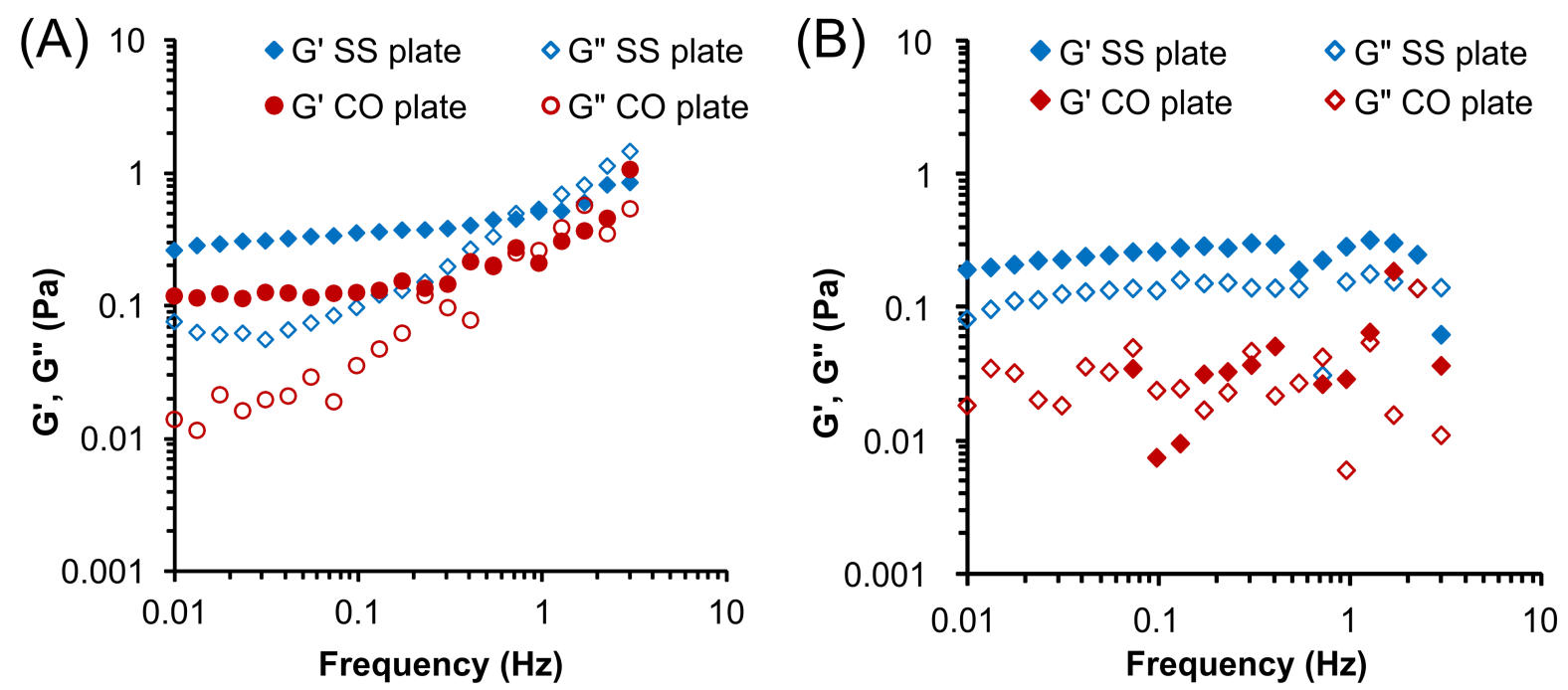

Figure 3.21: Comparison of different materials of bottom plates of the rheometer. Frequency sweeps of (A) actin and (B) BRB 80 buffer on stainless and chromium oxide bottom plates. Linear moduli $\left(G^{\prime}\right.$ and $\left.G^{\prime \prime}\right)$ are consistently higher on the stainless steel bottom plate for actin as well as buffer.

know the differences resulting from bottom plate material to selectively utilize them based on sample and experiment requirements.

\subsubsection{Case of increasing $G^{\prime}$ over time}

Time sweep experiments on microtubules that were polymerized in a tube and then pipetted onto the rheometer presented a curious problem. The linear shear moduli, $G^{\prime}$ and $G^{\prime \prime}$, of MTs kept increasing for about an hour before finally saturating to a plateau value, as shown in Fig. 3.22 A. Interestingly, placing the sample in rheometer and waiting for $1 \mathrm{hr}$ before starting the measurement directly gave saturated $G^{\prime}$ and $G^{\prime \prime}$ value. Fig. 3.22B shows experiments on actin, which also showed a similar trend of increase in shear moduli after pipetting onto the rheometer.

Control measurements were also performed on glycerol (data not shown), which showed a consistent value of loss modulus over time. Therefore, an increase in shear modulus over time is not an inherent error given by rheometer, but rather unique to the system of biopolymers. One possibility is that the filaments are randomized after pipetting and probably reorient during an hour prior to stabilization. Another possibility is that the observed increase in linear moduli may arise from line tension artifacts due to evaporation of sample (as discussed in Section 3.10.1). Unfortunately, we could not verify the exact nature of these artifacts. However, this observation once again validates the necessity to be aware of possible artifacts of rheometer, especially while working with soft samples. 

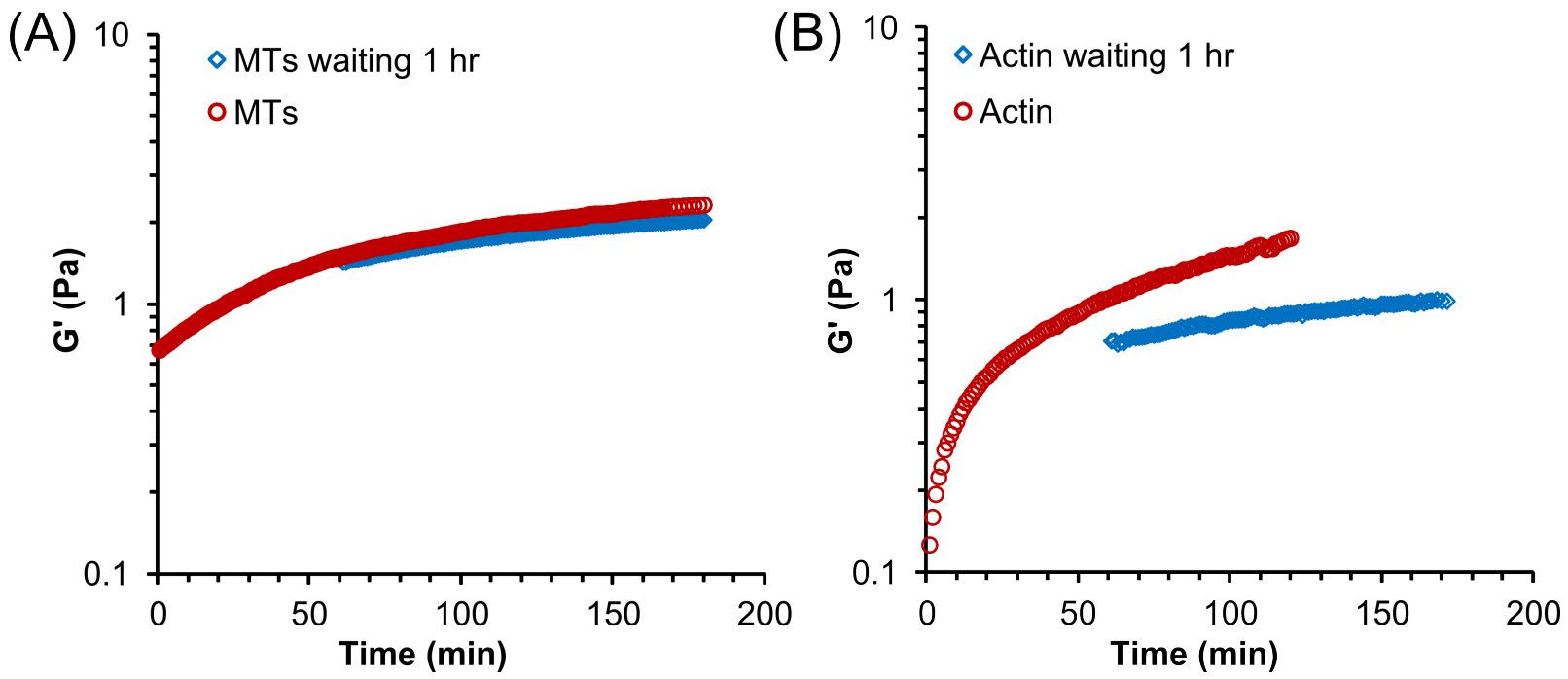

Figure 3.22: Increase in $G^{\prime}$ over time. (A) Time sweeps of microtubules, measured immediately after pipetting sample on rheometer and after $1 \mathrm{hr}$ waiting. The $G^{\prime}$ increased rapidly and reached a plateau value. Waiting for $1 \mathrm{hr}$ directly gave plateau $G^{\prime}$ values. (B) Similar behavior was observed for time sweeps of actin, with and without $1 \mathrm{hr}$ waiting.

\section{Bibliography}

[1] Malkin, A. I.; Malkin, A. Y.; Isayev, A. I. Rheology: concepts, methods, and applications; Chem Tec Publishing, 2006.

[2] Adams, N.; Lodge, A. Philosophical Transactions of the Royal Society of London. Series A, Mathematical and Physical Sciences 1964, 256, 149-184.

[3] Storm, C.; Pastore, J. J.; MacKintosh, F. C.; Lubensky, T. C.; Janmey, P. A. Nature 2005, 435, 191-194.

[4] Xu, J.; Wirtz, D.; Pollard, T. D. Journal of Biological Chemistry 1998, 273, 9570-9576.

[5] Gardel, M.; Nakamura, F.; Hartwig, J.; Crocker, J.; Stossel, T.; Weitz, D. Proceedings of the National Academy of Sciences of the United States of America 2006, 103, 1762-1767.

[6] Wang, N.; Tolić-Nørrelykke, I. M.; Chen, J.; Mijailovich, S. M.; Butler, J. P.; Fredberg, J. J.; Stamenović, D. American Journal of Physiology-Cell Physiology 2002, 282, C606-C616.

[7] Alcaraz, J.; Buscemi, L.; Grabulosa, M.; Trepat, X.; Fabry, B.; Farré, R.; Navajas, D. Biophysical journal 2003, 84, 2071-2079.

[8] Fabry, B.; Maksym, G. N.; Butler, J. P.; Glogauer, M.; Navajas, D.; Fredberg, J. J. Physical review letters 2001, 87, 148102.

[9] Mizuno, D.; Tardin, C.; Schmidt, C.; MacKintosh, F. Science 2007, 315, 370-373. 
[10] Janmey, P. A.; Weitz, D. A. Trends in biochemical sciences 2004, 29, 364-370.

[11] Discher, D. E.; Janmey, P.; Wang, Y.-I. Science 2005, 310, 1139-1143.

[12] Evans, E.; Yeung, A. Biophysical journal 1989, 56, 151.

[13] Zhelev, D. V.; Hochmuth, R. M. Biophysical journal 1995, 68, 2004.

[14] Rotsch, C.; Radmacher, M. Biophysical journal 2000, 78, 520-535.

[15] Wu, H.; Kuhn, T.; Moy, V. Scanning 1998, 20, 389-397.

[16] Janmey, P.; Peetermans, J.; Zaner, K.; Stossel, T.; Tanaka, T. Journal of Biological Chemistry 1986, 261, 8357-8362.

[17] Hinner, B.; Tempel, M.; Sackmann, E.; Kroy, K.; Frey, E. Physical Review Letters 1998, 81, 2614.

[18] Ruddies, R.; Goldmann, W.; Isenberg, G.; Sackmann, E. Biochemical Society transactions 1993, 21, 37S-37S.

[19] Shin, J.; Gardel, M.; Mahadevan, L.; Matsudaira, P.; Weitz, D. Proceedings of the National Academy of Sciences of the United States of America 2004, 101, 9636-9641.

[20] Gardel, M.; Shin, J.; MacKintosh, F.; Mahadevan, L.; Matsudaira, P.; Weitz, D. Physical review letters 2004, 93, 188102.

[21] Lieleg, O.; Claessens, M. M.; Bausch, A. R. Soft Matter 2010, 6, 218-225.

[22] Matsudalra, P. Trends in biochemical sciences 1991, 16, 87-92.

[23] Puius, Y. A.; Mahoney, N. M.; Almo, S. C. Current opinion in cell biology 1998, 10, 23-34.

[24] Lieleg, O.; Claessens, M. M.; Heussinger, C.; Frey, E.; Bausch, A. R. Physical review letters 2007, 99, 088102.

[25] Wagner, B.; Tharmann, R.; Haase, I.; Fischer, M.; Bausch, A. R. Proceedings of the National Academy of Sciences 2006, 103, 13974-13978.

[26] Lieleg, O.; Schmoller, K. M.; Cyron, C. J.; Luan, Y.; Wall, W. A.; Bausch, A. R. Soft Matter 2009, 5, 1796-1803.

[27] Tharmann, R.; Claessens, M.; Bausch, A. Physical review letters 2007, 98, 088103.

[28] Gardel, M.; Shin, J.; MacKintosh, F.; Mahadevan, L.; Matsudaira, P.; Weitz, D. Science 2004, 304, 1301-1305.

[29] Fernández, P.; Pullarkat, P. A.; Ott, A. Biophysical journal 2006, 90, 3796-3805. 
[30] Broedersz, C.; Storm, C.; MacKintosh, F. Physical review letters 2008, 101, 118103.

[31] Sharma, A.; Sheinman, M.; Heidemann, K.; MacKintosh, F. Physical Review E 2013, 88, 052705.

[32] Heidemann, K. M.; Sharma, A.; Rehfeldt, F.; Schmidt, C. F.; Wardetzky, M. Soft matter 2015, 11, 343-354.

[33] Hyman, A.; Drechsel, D.; Kellogg, D.; Salser, S.; Sawin, K.; Steffen, P.; Wordeman, L.; Mitchison, T. Methods in enzymology 1990, 196, 478-485.

[34] Hyman, A. Journal of Cell Science 1991, 1991, 125-127.

[35] Lin, Y.-C.; Koenderink, G. H.; MacKintosh, F. C.; Weitz, D. A. Macromolecules 2007, 40, 7714-7720.

[36] Yang, Y.; Bai, M.; Klug, W. S.; Levine, A. J.; Valentine, M. T. Soft matter 2013, 9, 383-393.

[37] Kuhlman, P. A. Cell motility and the cytoskeleton 2005, 61, 1-8.

[38] Kuriyama, R.; SAKAI, H. The Journal of Biochemistry 1974, 76, 651-654.

[39] Luduena, R. F.; Roach, M. C. Pharmacology \& therapeutics 1991, 49, 133-152.

[40] Mi, L.; Xiao, Z.; Hood, B. L.; Dakshanamurthy, S.; Wang, X.; Govind, S.; Conrads, T. P.; Veenstra, T. D.; Chung, F.-L. Journal of Biological Chemistry 2008, 283, 22136-22146.

[41] Phelps, K.; Walker, R. Biochemistry 2000, 39, 3877-3885.

[42] e Silva, M. S.; Depken, M.; Stuhrmann, B.; Korsten, M.; MacKintosh, F. C.; Koenderink, G. H. Proceedings of the National Academy of Sciences 2011, 108, 9408-9413.

[43] Kasza, K.; Broedersz, C.; Koenderink, G.; Lin, Y.; Messner, W.; Millman, E.; Nakamura, F.; Stossel, T.; MacKintosh, F.; Weitz, D. Biophysical journal 2010, 99, 1091-1100.

[44] Miller, R. K.; Vikstrom, K.; Goldman, R. D. The Journal of cell biology 1991, 113, 843-855.

[45] Kasza, K. E.; Broedersz, C. P.; Koenderink, G. H.; Lin, Y. C.; Messner, W.; Millman, E. A.; Nakamura, F.; Stossel, T. P.; MacKintosh, F. C.; Weitz, D. Biophysical Journal 2010, 99, 1091.

[46] Broedersz, C. P.; Storm, C.; MacKintosh, F. C. Physical Review Letters 2008, 101, 118103.

[47] Riedl, J.; Crevenna, A. H.; Kessenbrock, K.; Yu, J. H.; Neukirchen, D.; Bista, M.; Bradke, F.; Jenne, D.; Holak, T. A.; Werb, Z.; Sixt, M.; Wedlich-Soldner, R. Nature methods 2008, 5, 605-607. 
[48] Hell, S. W.; Wichmann, J. Optics letters 1994, 19, 780-782.

[49] Paredes, J.; Michels, M. A.; Bonn, D. Physical review letters 2013, 111, 015701.

[50] Ikeda, A.; Berthier, L.; Sollich, P. Physical review letters 2012, 109, 018301.

[51] Johnston, M. T.; Ewoldt, R. H. Journal of Rheology 2013, 57, 1515-1532.

[52] Schweizer, T. Applied Rheology 2004, 14, 197-201.

[53] Hellström, L. H. O.; Samaha, M. A.; Wang, K. M.; Smits, A. J.; Hultmark, M. Measurement Science and Technology 2015, 26, 015301. 



\section{Chapter 4}

\section{DNA force sensors in cells}

Reconstituted in vitro experimental systems are considered to be simple in terms of the number of components. These systems can however be complicated to interpret, as discussed in the previous chapter. It is evidently essential to walk the tight rope, seeking best of both, in vitro and in vivo systems, in experiments. We therefore, tested attachment of the DNA force sensor to actin filaments in human mesenchymal cells (hMSCs). The results of our preliminary experiments are described in this short chapter. 
As actin is the most dynamic among the cytoskeletal filaments, we chose to attach the DNA force sensor to filamentous actin in cells. Lifeact-Halotag fusion protein was purified from E.coli, as described in Section 6.5. Different methods were tested for transfection of the DNAprotein complex in cells. These included electroporation, transfection with a chemical reagents such as Viafect and Saponine. The detailed protocols for each method are given in Chapter 6. Briefly, Lifeact-Halotag fusion protein was incubated with a mixture of fluorescent DNA sensor (without the quencher strand) and Haloligand. The attachment of the fusion protein to actin in cells should result in fluorescently marked actin filaments. The cells did not survive the Saponine treatment and therefore no images are shown from this method. Fig. 4.1A, B shows fluorescent and phase contrast images of cells at 0 and $24 \mathrm{hrs}$ after electroporation and transfection with Viafect, respectively. The cells were fluorescent on imaging immediately after transfection (time 0) with both the methods; albeit with varying efficiencies. However two main issues surfaced from these experiments. Importantly, although the cells were fluorescent we could not see filamentous actin structures, implying that the fluorescence did not arise from the specific attachment of the fusion protein to its target. Another issue was concerning the fate of the protein-DNA sensor complex. Electroporated cells, when imaged after $24 \mathrm{hrs}$, showed a weak remnant fluorescence, while the fluorescent complex appeared to be localized in vesicles with passage of time in the case of cells transfected with Viafect. The localization is most likely an effect of triggering of defense mechanisms of the cells; possibly due to targeting of the protein-DNA mixture directly into the nucleus.

We therefore also tested Fuse-It-P as a transfection agent, which is specifically designed to deliver proteins into the cytoplasm of cells. A Haloligand labeled with TMR dye was used as a fluorescent marker, instead of the DNA sensor, in these experiments. Nevertheless, in these experiments as well, the fluorescence remained homogeneously distributed in the cytoplasm and weakened drastically over time. No filamentous structures were observed, as shown in Fig. 4.1.

The preliminary cell experiments leave scope for optimization of many parameters. A range of concentrations of protein-DNA mixtures must be tested to find the combination that works best for the cells. Importantly, control tests must be performed with the introduction of just a fluorescent labeled Haloligand into cells, without the fusion proteins. This would clarify if the homogeneous fluorescence in cells arises purely from the ligand. Lastly, expressing the fusion proteins in cells, followed by transfection of the DNA sensor could be tested as an alternative to the introduction of large protein-DNA complexes externally. Although, we could not do the needful, due to shortage of time, these experiments set the stage for improved future experiments. 

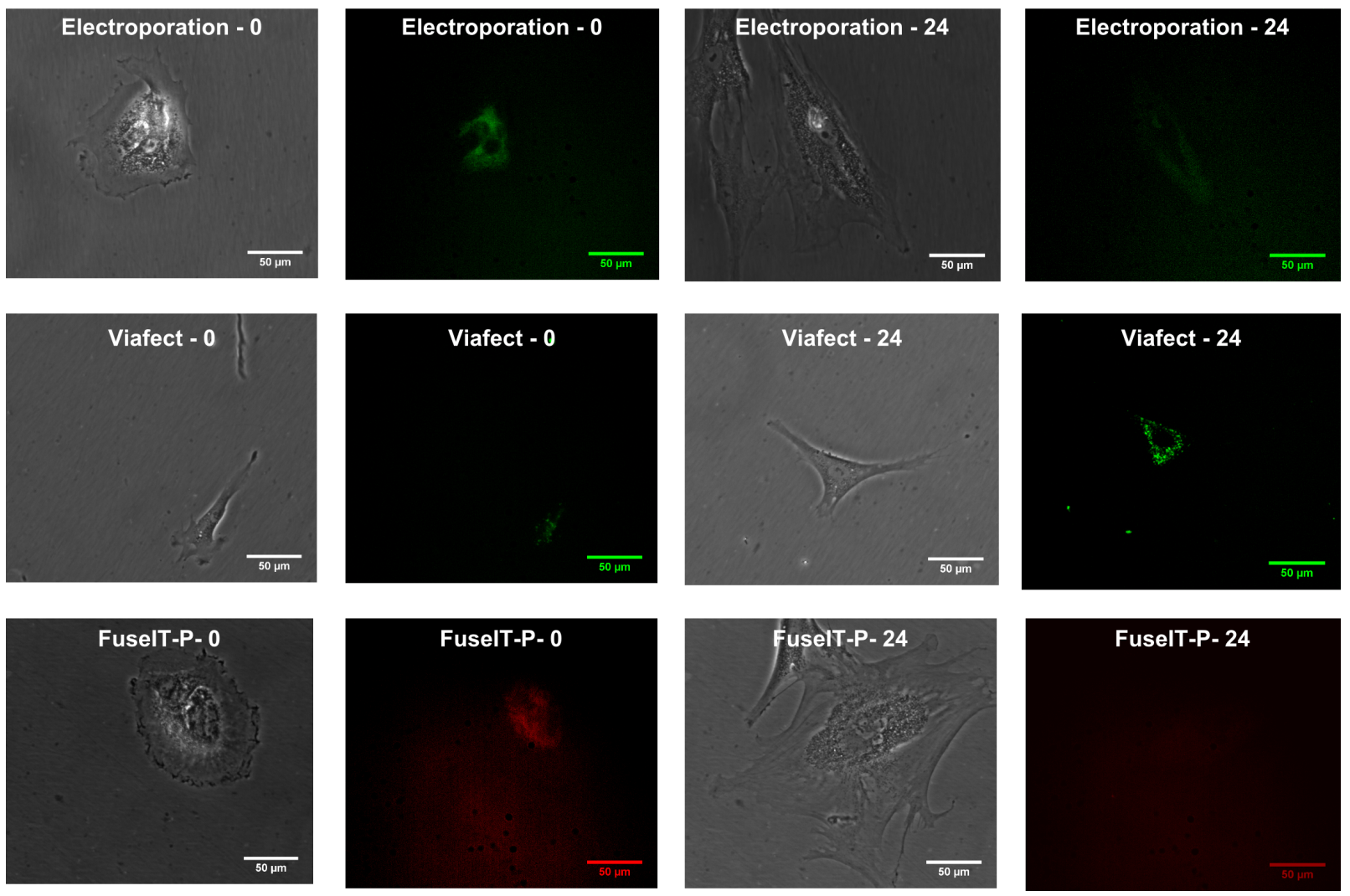

Figure 4.1: Introducing DNA force sensor in cells. Human mesenchymal cells (hMSCs) were transfected with Lifeact-Halotag fusion protein combined with fluorescent DNA sensor. Different methods of transfection were used and images were recorded for $24 \mathrm{hrs}$. Images of cells in fluorescence and phase contrast recorded at 0 and 24 hrs after (A) electroporation, (B) transfection with Viafect and (C) transfection with Fuselt-P, which specifically delivers proteins in cytoplasm. Scale bar is $50 \mu \mathrm{m}$. The cells showed homogeneous fluorescence when imaged immediately after transfection with the different methods. The fluorescence weakens for electroporated and Fuse-lt-P treated cells, while Viafect treated cells show localization of fluorescence in vesicles. Note that fluorescent DNA was not used for FuselT-P transfection; TMR dye labeled Haloligand was used as a fluorescent marker instead. 



\section{Chapter 5}

\section{Summary and Outlook}

The following chapter summarizes the experimental observations described in the thesis. $A$ short outlook regarding possible future experiments is included in this chapter. 
The presented work introduces a DNA hairpin based force sensor that is sensitive to forces of the order of $10 \mathrm{pN}$ or more. The unfolding of the hairpin directly translates to a visual fluorescence signal from an incorporated fluorescence-quencher pair, which undergoes FRET. Furthermore, the sensor design is such that it can be easily modified as per experimental requirements. This is useful in terms of modifying the DNA sequence or length to change the forces required for unfolding the hairpin. Dual color FRET experiments can also be performed by replacing the quencher with an appropriate fluorescent acceptor dye. The working of the DNA sensor was demonstrated in bulk and at a single molecule level through control experiments.

The in vitro demonstration of force sensing on immobilized DNA hairpins proved challenging due to technical issues and inadequate information regarding orientation of the sensor. Moreover, as immobilization would limit force sensing to a 2D system, we designed a 3D experimental system that is biologically more relevant. Reconstituted networks of microtubules, crosslinked by the DNA sensor, serve as a model for composite networks resembling those found in cells. Yet, such a system is not well studied and therefore, remains less understood. Our dual goal behind the choice of networks of microtubules crosslinked with DNA as an experimental system was to characterize rheology of these networks, along with force sensing experiments.

Experimental trials of perturbing these networks with a glass needle revealed some artifacts, necessitating optimization of the force application method. A possible, alternative method would be using dual bead optical trap to shear the in vitro networks. Meanwhile, classical linear and nonlinear rheology tests were conducted in parallel, to understand mechanics of the networks. A rather surprising observation was that rheology and microscopy methods proved insufficient to gather evidence of crosslinking of MTs, irrespective of the strategy used for crosslinking. Furthermore, additional problems such as deleterious effects of chemical crosslinker, sulfo-SMCC, on MT length or artifacts from instrumentation led to unforseen problems in these experiments. While the credibility for crosslinking strategies was salvaged in case of actin, it became generally clear that in vitro experiments are not exactly a 'simple' representation of the cellular network. Although these networks consist of fewer components, the system can prove to be extremely complicated at a molecular level. Moreover, as stiff rods cannot easily bend like actin, composite networks of stiff rods are especially sensitive to parameters such as filament length. This might provide an explanation regarding the difficulties in crosslinking microtubules; a non-issue in case of actin. Therefore, force sensing experiments can be combined with crosslinking studies of actin networks in future, to complete the in vitro experiments on the sensor. Actin can also be treated as stiff rods, depending on its length, thereby extending the possibility to compare experimental data with simulations of stiff rods and flexible crosslinkers.

Preliminary experimental data showed that the DNA sensor, complexed with the LifeactHalotag fusion protein, can be introduced into cells through different methods of transfection. Some control experiments and optimization of concentrations is required for achieving specific binding to actin filaments in cells. Furthermore, expressing the fusion protein in cells and transfecting only the small sized DNA-ligand mixture could ensure better uptake and prolonged survival of the DNA sensor in cells.

In conclusion, through this study, we developed a new tool for force sensing in 3D cytoskeletal networks that supports a unified approach to study multiple cellular processes together. The 
construction and analysis of in vitro networks gave an insight into the loopholes in artificial networks and also highlights basic differences in semi-flexible and rigid rod networks. Although our contribution is merely a grain in the sandbox of network mechanics, we believe that it is an important step to understand the fundamental problems while dealing with different types of polymers. This will allow us to choose the polymer type wisely, depending on the experimental system. Lastly, with the in vivo experiments, we demonstrate the possibility of using the force sensor in cells in the near future. 



\section{Chapter 6}

\section{Materials and methods}

Materials were obtained from Sigma-Aldrich Chemie Gmbh (Munich, Germany), unless specified otherwise. 


\subsection{Preparation of DNA}

\subsubsection{DNA sensors}

The sequences of DNA sensor strands were designed online on NUPACK webpage and obtained commercially (Integrated DNA Technologies, Leuven, Belgium). ${ }^{1}$ Lyophilized powders were resuspended in DNA buffer and aliquots were stored at $-20^{\circ} \mathrm{C}$. The design of sensor is explained in Section 2.5 and sequences of strands are given below.

\section{Hairpin strand, $\mathrm{H}$}

CGCCCTGAAAGCACGCAGCGGCGAACCGGAGAGTGTTAGAGACACGGTTCGCGCGCGGG CACGCGGCTTGCG

\section{Complementary arm with fluorophore, $F$}

In reversible sensor design, the strands bears the fluorophore, Alexa 488. CGCTGCGTGCTTTCAGGGCG

\section{Complementary arm with quencher, $\mathrm{Q}$}

In reversible sensor design, the strands bears the quencher, lowa black dark quencher. CGCAAGCCGCGTGCCCGCGC

\section{Fluorophore strand, F}

In the irreversible sensor the fluorophore is not incorporated in the hairpin but attached to this strand.

TGTCTCTAACACTCTCCGGTTCGC

\section{Quencher strand, Q}

In the irreversible sensor the quencher is not incorporated in the hairpin but attached to this strand.

GAGAGTGTTAGAGACA

Control strand for irreversible sensor, C

ACAGAGATTGTGAGAGGCCAAGCG

Control strand for reversible sensor, C

TGTCTCTAACACTCTCCGGTTCGC 


\section{Polyacrylamide gel electrophoreses (PAGE)}

(A)

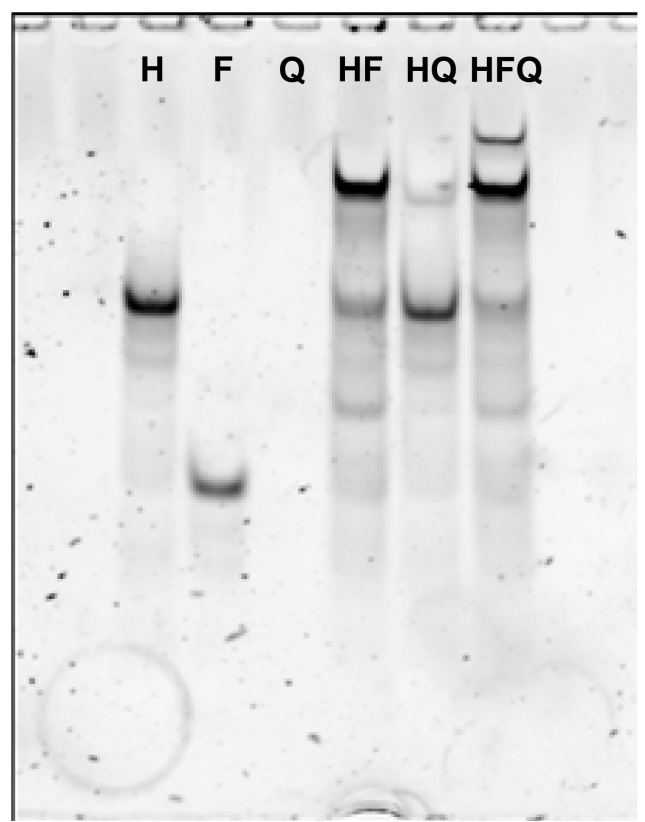

(B)

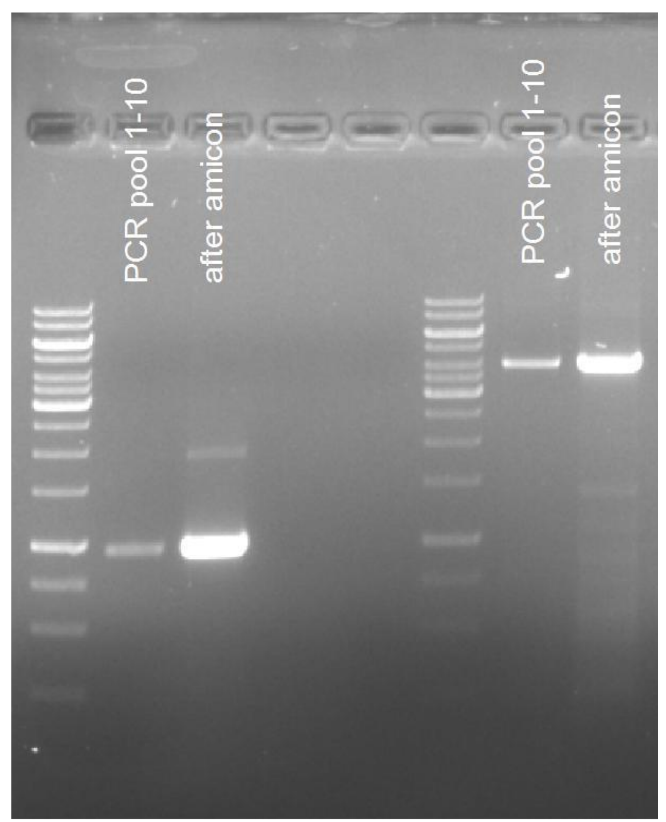

Figure 6.1: DNA strands on gels. (A) Polyacrylamide gel showing hybridization of $100 \mathrm{nM}$ strands of the reversible DNA sensor. (B) DNA fragments of $1 \mathrm{~kb}$ and $4 \mathrm{~kb}$ on an agarose gel after PCR are shown in lanes 2 and 7 , respectively. Lanes 3 and 8 show $1 \mathrm{~kb}$ and $4 \mathrm{~kb}$ fragments after purification and concentration.

Hybridization and sizes of DNA strands were tested on 20\% Novex TBE gel (Life Technologies $\mathrm{GmbH}$, Darmstadt, Germany) run at $250 \mathrm{~V}$ for $40 \mathrm{~min}$ in TBE buffer (SERVA Electrophoresis $\mathrm{GmbH}$, Heidelberg, Germany). GeneRuler ultra low range DNA ladder (Life Technologies GmbH, Darmstadt, Germany) was run as reference to estimate size of DNA. The gel was stained with 1 to 10000 diluted SYBR Gold Nucleic Acid Gel Stain (Life Technologies $\mathrm{GmbH}$, Darmstadt, Germany) for approximately 30 min and imaged using UV filter in Intas ECL Chemocam imager (INTAS Science Imaging Instruments GmbH, Sartorius, Goettingen, Germany). A gel showing hybridization of strands of the reversible DNA sensor is shown in Fig. 6.1A. The lane with HFQ shows a fraction of unhybridized HF strands; responsible for background fluorescence in the spectrophotometer.

\section{DNA purification}

DNA constructs were purified with Amicon Ultra- $0.5 \mathrm{~mL}$ Centrifugal Filters with $3 \mathrm{kDa}$ cut-off (Merck Chemicals GmbH, Hessen, Germany), following company specified protocol.

\section{Spectrophotometer experiments}


Control experiments were performed to test working of the DNA sensors, which are described in Section 2.5. Bulk experiments were done on AMINCO-Bowman Series 2 spectrophotometer (Thermo Electron Scientific Instruments Corporation, Madison, WI, USA). DNA buffer was used as negative control. DNA sensor strands ( $F, Q$ and $C)$ were added sequentially and hybridized in situ in quartz cuvettes (Hellma Analytics, Müllheim, Germany). Final DNA concentration was $25 \mathrm{nM}$ in a final volume $100 \mu \mathrm{l}$. DNA strands were labeled with fluorophore Alexa 488 and measured at an excitation wavelength of $494 \mathrm{~nm}$ and an emission wavelength of $520 \mathrm{~nm}$.

\section{Single molecule experiments}

$1 \mathrm{nM}$ of HF and HFQ strands (reversible DNA sensor) were spin coated at $8000 \mathrm{rpm}$ for $40 \mathrm{~s}$ on plasma cleaned coverslips. Confocal scans of single molecules were obtained on the commercial confocal system Microtime 200 (PicoQuant, Berlin, Germany).

Details of the instrumentation are as follows. A modified Olympus IX-71 microscope (Olympus Deutschland, Hamburg, Germany) was used and samples were scanned at a speed of $5 \mathrm{~ms}$ per $100 \mathrm{~nm}$ pixel by using a three-axis piezo stage. A pulsed diode laser $(\lambda=480 \mathrm{~nm})$ with a pulse width of $100 \mathrm{ps}$ full-width at half-maximum was operated at a pulsing frequency of $20 \mathrm{MHz}$. The excitation beam was then reflected by using a dichroic mirror to guide it into the side port of the microscope. The excitation power used was around $10-25 \mathrm{~kW} \mathrm{~cm}{ }^{2}$. A high numerical aperture objective was used to excite the samples (UAPON 100XOTIRF, 1.49 N.A., Olympus) and to collect the fluorescence emission. The emission light was then passed through the dichroic mirror and focused onto the active area (approximately $75 \mu \mathrm{m}$ ) of a single-photon counting module (SPCMAQR-13, PerkinElmer). A band-pass filter (BrightLine HC 525/45, Semrock) was used before the detector to block the back-scattered laser light. The photons detected were counted using a multichannel picosecond event timer and time-correlated single-photon counting (TCSPC) module (HydraHarp 400, PicoQuant). The collected photon data was processed by using custom written Matlab routines in order to obtain intensity and lifetime images. Further technical details are given elsewhere. ${ }^{2}$

\subsubsection{Long DNA fragments}

We used DNA of different lengths for crosslinking microtubules, in order to understand the dependence of network mechanics on crosslinker length. The results are shown in Section 3.5.2.

\section{Polymerase chain reaction (PCR)}

$1 \mathrm{~kb}$ and $4 \mathrm{~kb}$ fragments were amplified from plasmid UNC 104 using thiol modified primers and Phusion High-Fidelity DNA Polymerase (New England Biolabs GmbH, Ipswich, MA). ${ }^{3}$

PCR programs used for amplification of $1 \mathrm{~kb}$ and $4 \mathrm{~kb}$ DNA fragments are given in Table. 6.1. 


\begin{tabular}{|c|cc|cc|}
\hline Step & $\mathbf{1} \mathbf{k b}$ & $\mathbf{1} \mathbf{k b}$ & $\mathbf{4} \mathbf{k b}$ & $\mathbf{4} \mathbf{k b}$ \\
\hline 1 & $98^{\circ} \mathrm{C}$ & $30 \mathrm{~s}$ & $98^{\circ} \mathrm{C}$ & $30 \mathrm{~s}$ \\
2 & $95^{\circ} \mathrm{C}$ & $10 \mathrm{~s}$ & $95^{\circ} \mathrm{C}$ & $10 \mathrm{~s}$ \\
3 & $50^{\circ} \mathrm{C}$ & $30 \mathrm{~s}$ & $58^{\circ} \mathrm{C}$ & $30 \mathrm{~s}$ \\
4 & $72^{\circ} \mathrm{C}$ & $30 \mathrm{~s}$ & $72^{\circ} \mathrm{C}$ & $2 \mathrm{~m}$ \\
5 & $72^{\circ} \mathrm{C}$ & $10 \mathrm{~m}$ & $72^{\circ} \mathrm{C}$ & $10 \mathrm{~m}$ \\
6 & $4^{\circ} \mathrm{C}$ & Hold & $4^{\circ} \mathrm{C}$ & Hold \\
\hline
\end{tabular}

Table 6.1: PCR programs

\section{Agarose gel electrophoreses}

The DNA fragments were run on $1 \%$ agarose gel (CarlRoth $\mathrm{GmbH}+$ Co.KG) with $6 \mathrm{X}$ loading dye (Life Technologies $\mathrm{GmbH}$, Darmstadt, Germany). GeneRuler 1 kb DNA Ladder (Life Technologies GmbH, Darmstadt, Germany) was run as reference for sizes. Midori Green (Biozym Scientific GmbH, Oldendorf, Germany) was added to gels in a dilution of 1 to 10000 while casting. Images were recorded using UV filter of Intas ECL Chemocam imager (INTAS Science Imaging Instruments $\mathrm{GmbH}$, Sartorius, Göttingen, Germany). A gel showing $1 \mathrm{~kb}$ and $4 \mathrm{~kb}$ DNA fragments before and after purification is shown in Fig. 6.1B.

\section{DNA purification}

PCR amplified DNA fragments were purified using QIAquick PCR Purification Kit (QIAGEN GmbH, Hilden, Germany), as per protocol specified in product manual.

\subsection{Immobilization protocols}

The DNA sensor was immobilized on a coverslip to test the opening of hairpins on force application in a minimum component system.

\subsubsection{DNA immobilization on coverslips}

DNA with thiol ends was attached to coated coverslips using a protocol, modified according to our requirements, from Ref. 4. Main steps of the protocol are described below.

1. Glass coverslips were cleaned in plasma cleaner (Harrick Plasma, Ithaca, NY, USA) for $15 \mathrm{~min}$.

2. Coverslips were immersed in absolute ethanol $(250 \mu \mathrm{l})$ and ultrasonicated for $5 \mathrm{~min}$. 
3. Silanization was done by incubating coverslips in a $2 \%$ solution of 3 -aminopropyl dimethyl ethoxysilane (APTES) in ethanol for $30 \mathrm{~min}$.

4. Silanized coverslips were washed twice with ethanol, followed by ultrapure water and air dried with stream of nitrogen.

5. Bake at $80^{\circ} \mathrm{C}$ for $1 \mathrm{hr}$.

6. Coverslips were wetted with HEPES buffer $(\mathrm{pH} 8)$ for $1 \mathrm{hr}$ followed by incubation with 10 mM sulfo-SMCC (Life Technologies GmbH, Darmstadt, Germany) sandwiched between two coverslips.

7. The coverslips were separated and washed in ultrapure water and air dried with a stream of nitrogen.

8. Thiol modified DNA strands (Integrated DNA Technologies, Leuven, Belgium) were reduced with 20X Bond-Breaker TCEP solution (Life Technologies GmbH, Darmstadt, Germany) for 30 min at ambient temperature.

9. 2-5 $\mu$ l of DNA was spotted on coated coverslip and incubated for $1 \mathrm{hr}$ in water saturated environment. The concentration of DNA used depended on experiment requirements and ranged from $10 \mathrm{nM}-1 \mu \mathrm{M}$.

10. Controls included spotting with L-cysteine to block maleimide groups from sulfo-SMCC and then adding thiol DNA to same spot and/or using DNA with biotin end to check for unspecific binding.

11. Coverslips were washed carefully in PBS buffer and air dried in stream of nitrogen.

\subsubsection{Coating AFM cantilever with neutravidin}

AFM experiments to unfold DNA hairpins are described in Section 2.6.2. Cantilevers were coated using a modified protocol from Ref. 5,6. Olympus Biolever RC150VB cantilevers (Olympus Europa SE \& Co. KG, Hamburg, Germany), made of silicon nitride were used with a nominal resonance frequency of $37 \mathrm{kHz}$ (in air) and a nominal spring constant $30 \mathrm{pN} / \mathrm{nm}$. Cantilevers were immersed in acetone (CHROMASOLV grade) for $5 \mathrm{~min}$ followed by UV irradiation for $30 \mathrm{~min}$. Subsequently, cantilevers were incubated overnight in $0.5 \mathrm{mg} / \mathrm{ml}$ biotinylated BSA at room temperature. Cantilevers were washed the following day 6 times with PBS ( $\mathrm{pH} 7)$ and incubated with $0.5 \mathrm{mg} / \mathrm{ml}$ neutravidin (Life Technologies GmbH, Darmstadt, Germany) for 1 $\mathrm{hr}$. The cantilevers were ready to use after rinsing with PBS to wash off excess neutravidin. 


\subsubsection{Coating beads with neutravidin}

Flow experiments to unfold DNA hairpins are described in Section 2.6.3. Beads were attached to the DNA strands using a protocol described below.

Biotin used: 6-((6-((biotinoyl)amino)hexanoyl)amino)hexanoic acid, succinimidyl ester) 50 $\mu \mathrm{l}$ carboxylated silica beads $(1.5 \mu \mathrm{m}$; Kisker Biotech $\mathrm{GmbH} \&$ Co. KG, Steinfurt, Germany) solution was diluted in $950 \mu \mathrm{l}$ phosphate buffer. Beads were centrifuged at $7800 \mathrm{~g}$ for $10 \mathrm{~min}$ to remove any impurities and pellet resuspended in $800 \mu \mathrm{l}$ phosphate buffer. $100 \mu \mathrm{l} \mathrm{N}$-(3Dimethylaminopropyl)-N-ethylcarbodiimide hydrochloride (EDC) and $100 \mu \mathrm{l}$ Biotin-XX, SE (1 $\mathrm{mg} / \mathrm{ml}$ ) were incubated for $1 \mathrm{hr}$ while shaking. The beads were centrifuged at $7800 \mathrm{~g}$ for 10 min followed by resuspension in $1 \mathrm{ml}$ buffer twice and stored at $40^{\circ} \mathrm{C}$.

$25 \mu \mathrm{l}$ of biotinylated beads were diluted in $55 \mu \mathrm{l}$ phosphate buffer, along with addition of $10 \mu \mathrm{l}$ glycine and $10 \mu \mathrm{l}$ neutravidin. After $1 \mathrm{hr}$ incubation (while shaking) at $7800 \mathrm{~g}$ for $10 \mathrm{~min}$, the pellet was resuspended in $100 \mu \mathrm{l}$ phosphate buffer. The centrifugation step was repeated and beads stored at $40^{\circ} \mathrm{C}$ until further use.

\subsection{Preparation of microtubules}

Microtubules were polymerized in vitro for assembling artificial networks, as described in Chapter 3. The protocols for preparation and handling of MTs are given below.

\subsubsection{Reconstitution and storage of tubulin}

Porcine brain tubulin $(10 \mathrm{mg})$ was obtained commercially as lyophilized powder (Cytoskeleton, Inc., Denver, CO, USA). Tubulin was reconstituted to $20 \mathrm{mg} / \mathrm{ml}$ stock with General Tubulin Buffer (80 mM PIPES, pH 6.9, 2 mM MgCl $2,0.5$ mM EGTA), also obtained from Cytoskeleton, Inc., Denver, CO, USA. Aliquots of reconstituted tubulin were snap frozen in liquid nitrogen and stored at $-80^{\circ} \mathrm{C}$.

\subsubsection{Polymerization of MTs}

Microtubules were polymerized in BRB 80 buffer with $1 \mathrm{mM} \mathrm{GTP}, 2 \mathrm{mM} \mathrm{MgCl}, 10 \mu \mathrm{M}$ taxol and porcine brain tubulin at $37^{\circ} \mathrm{C}$ for 30 minutes. Final concentrations of tubulin used for rheology experiments was $2 \mathrm{mg} / \mathrm{ml}(20 \mu \mathrm{M})$, unless specified otherwise. Dilutions of polymerized MTs for microscopy were made in BRB 80 buffer containing $10 \mu \mathrm{M}$ taxol.

Some variations in protocol have been used in certain experiments as per requirements. Polymerization of labeled microtubules was done by mixing tetramethylrhodamine (TMR) labeled tubulin with unlabeled tubulin in a ratio of 1 to 5 . Similarly, biotinylated microtubules were polymerized by adding biotin labeled tubulin to unlabeled tubulin during polymerization. 
Short microtubules were polymerized by incubating a mixture of unlabeled tubulin with Nethylmaleimide (NEM) labeled tubulin (3:2) and 1 mM GMPCPP (Jena Bioscience GmbH, Jena, Germany) for 30 minutes at $37^{\circ} \mathrm{C}$.

\subsubsection{Cleaning MTs}

A glycerol cushion was prepared by mixing $60 \%$ glycerol in BRB 80 buffer and $10 \mu \mathrm{M}$ taxol. MTs were loaded gently on top without mixing and spun-down in air driven ultracentrifuge (Beckman Coulter, Inc., Palo Alto, CA) for $5 \mathrm{~min}$ at $10 \mathrm{psi}$. The pellet was resuspended in taxol containing BRB 80 buffer.

\subsubsection{DETA coating of coverslips}

Protocol for coating coverslips with a positively charged silane to attach negatively charged microtubules is described below.

1. Coverslips were cleaned for $15 \mathrm{~min}$ in a plasma cleaner.

2. Plasma cleaned coverslips were then silanized with $0.1 \%$ solution of 3-[2-(2-Aminoethylamino)ethylamino]propyl-trimethoxysilane (DETA) in ultra-pure water for $5 \mathrm{~min}$ and sonicated.

3. The next step was washing twice with Millipore water and sonication for 5 minutes.

4. Coverslips were dried at $70^{\circ} \mathrm{C}$ for approximately $1 \mathrm{hr}$.

\subsubsection{Microscopy of MTs}

Chambers of approximately $10 \mu \mathrm{l}$ volume were prepared by using double-sided tape of 100 $\mu \mathrm{m}$ thickness between glass slide and DETA coated coverslips. Polymerized MTs were diluted in taxol buffer as described above and flushed into the chambers. After incubation of $5 \mathrm{~min}$, antibleach solution was flushed in through one end and filter paper placed at other end to replace unattached MT solution by capillary action. Antibleach solution consists of BRB 80 buffer containing $10 \mathrm{mM}$ dithiotreitol (DTT), $0.08 \mathrm{mg} / \mathrm{ml}$ catalase $40,0.1 \mathrm{mg} / \mathrm{ml}$ glucose oxidase and $10 \mathrm{mM}$ glucose. Samples were imaged on an epifluorescence microscope, Axiovert 200 (Carl Zeiss Microlmaging GmbH, Jena, Germany) using an oil immersion objective (EC PlanNeofluar 100x/1.3, Carl Zeiss Microlmaging GmbH, Jena, Germany). Images were recorded with a digital CCD camera (CoolSnap ES, Roper Scientific, Martinsried, Germany) using a software Winspec (Roper Scientific, Martinsried, Germany). 
(A)

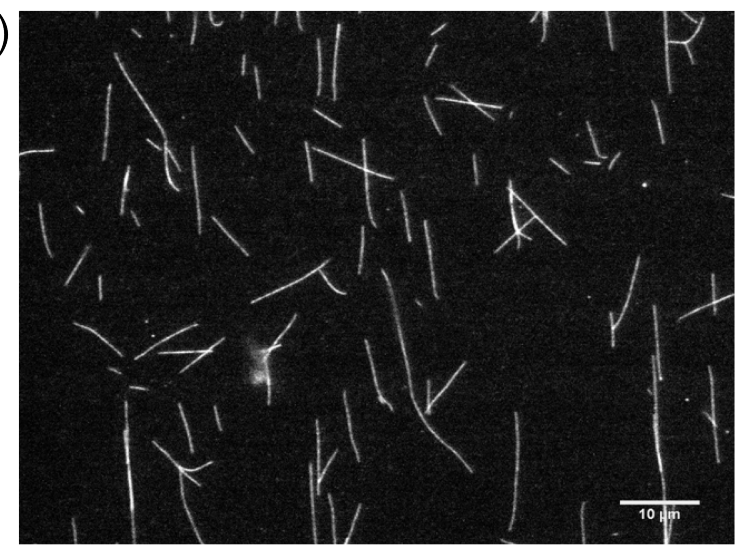

(B)

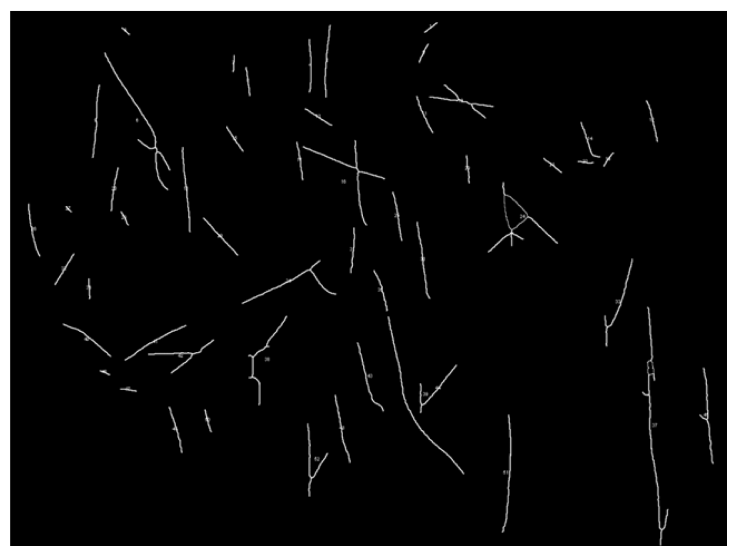

Figure 6.2: Image analysis routine. Raw image of MTs is shown in (A). Image processing was done in Fiji by thresholding with MaxEntropy, followed by skeletonization and selection of particles above a minimum length of $645 \mathrm{~nm}$ and circularity between 0 and 0.1 . MTs at edges were excluded as they went out of the field of view. The image after analysis routine is shown in $(B)$.

\subsubsection{Image analysis for length distribution of MTs}

Images of MTs treated with sufo-SMCC and maleimide dye were analyzed for their length distribution using Fiji software. The raw image of MTs is shown in Fig. 6.2 A. Image processing was done by thresholding using 'MaxEntropy' followed by 'skeletonization'. Using the Analyze particles' tool, a minimum length threshold of $0.645 \mathrm{~m}$ and circularity between $0-0.1$ were selected to avoid measuring tubulin aggregates. The image after analysis routine is shown in Fig. 6.2B. As the skeletonization step reduces objects to single pixel width, fitting an ellipse to straight MT will result in minor axis value of 1 . Individual MTs were sorted from clusters of MTs by neglecting those with minor axis values greater than 4 . The lengths for long and looped MTs were measured manually.

\subsection{Preparation of actin}

Actin filaments were polymerized in vitro for assembling artificial networks, as described in Chapter 3. The protocols for preparation and handling of actin are given below.

\subsubsection{Reconstitution and storage of actin}

G-actin (1 mg) from rabbit skeletal muscle was purchased as lyophilized powder (Hypermol EK, Bielefeld, Germany). A stock of $4 \mathrm{mg} / \mathrm{ml}$ actin was prepared by reconstituting lyophilized actin in ultra-pure water. The stock solution contains $95.2 \mu \mathrm{M}$ actin, $8 \mathrm{mM}$ Tris- $\mathrm{Cl}(\mathrm{pH}$ 8.2), 1.6 
mM ATP, $2 \mathrm{mM}$ (DTT), $0.4 \mathrm{mM} \mathrm{CaCl} 2$ and $0.8 \%$ disaccharides. Aliquots were snap frozen in liquid nitrogen and stored at $-80^{\circ} \mathrm{C}$.

Monomix (Hypermol EK, Bielefeld, Germany) is used as a dilution buffer to obtain the required actin concentration. It consists of $2 \mathrm{mM}$ Tris- $\mathrm{Cl}(\mathrm{pH} 8.2), 0.4 \mathrm{mM} \mathrm{ATP}, 0.1 \mathrm{mM}$ $\mathrm{CaCl}_{2}$ and $0.5 \mathrm{mM} \mathrm{DTT}$. Small aliquots of $50 \mathrm{X}$ Monomix were stored at $80^{\circ} \mathrm{C}$ and diluted to $1 \mathrm{X}$ with millipore water, prior to use.

Polymix (Hypermol EK, Bielefeld, Germany) is used as a polymerization buffer to induce actin polymerization. It consists of $1 \mathrm{M} \mathrm{KCl}, 0.1 \mathrm{M}$ imidazole $(\mathrm{pH} 7.4), 10 \mathrm{mM}$ ATP and 20 $\mathrm{mM} \mathrm{MgCl}$. Aliquots of $10 \mathrm{X}$ Polymix were stored at $80^{\circ} \mathrm{C}$ and thawed on ice, prior to use.

\subsubsection{Polymerization of actin}

Actin was diluted to a final concentration of $1 \mathrm{mg} / \mathrm{ml} \mathrm{G}$-actin (stock $4 \mathrm{mg} / \mathrm{ml}$ ) with Monomix buffer. 10X Polymix was added in 1 to 9 ratio to the mix to get a final concentration of $1 \mathrm{X}$ Polymix. The mixture was incubated at $23^{\circ} \mathrm{C}$ for $1 \mathrm{hr}$ for polymerization of F-actin.

\subsubsection{Microscopy of actin}

Actin was labeled by incubating a mixture of $10 \mu \mathrm{l}$ actin, $89 \mu \mathrm{l} 1 \mathrm{X}$ Polymix buffer and $1 \mu \mathrm{l}$ Atto 647 with phalloidin (ATTO-TEC GmbH, Siegen, Germany) for $1 \mathrm{hr}$.

Labeled actin was further diluted as per requirement and pipetted onto DETA coated coverslip (preparation described in Section 6.3). After incubating for $5 \mathrm{~min}$, the fluid was gently washed with $1 X$ Polymix buffer. The coverslip was inverted onto a slide with $3 \mu \mathrm{l}$ mixture of Mowiol and Dabco as embedding medium. The medium consists of glycerin $(6 \mathrm{~g})$, polyvinilalcohol 4-88 $(2.4 \mathrm{~g})$, Tris Buffer $200 \mathrm{mM} \mathrm{pH} 8.5(12 \mathrm{ml}$ with $\mathrm{HCl})$, water $(6 \mathrm{ml})$, 1,4-diazobicyclo[2.2.2]octane $2.5 \% \mathrm{w} / \mathrm{v}(0.6 \mathrm{~g})$.

Actin filaments were imaged with confocal and stimulated emission depletion microscopy (STED) setup. In STED microscopy, the diffraction-limited excitation focal spot is overlapped with a donut-shaped laser on the default laser. This laser has the ability to switch off the fluorescent molecules in the periphery of the excitation spot, confining the fluorescent area to donut center. In our experiments, the excitation laser originated in a Chameleon Compact OPO-Vis unit (Coherent Inc., Santa Clara, CA USA), converting the IR light of a Ti:Sa laser in the visible $590 \mathrm{~nm}$ light. The excitation laser, pulsed at $80 \mathrm{MHz}$, triggers the $775 \mathrm{~nm}$ STED laser (Onefive $\mathrm{GmbH}$, Zurich, Switzerland). The delay between excitation and STED pulses was set by an home-made electronic delay unit. The donut-shaped depletion spot was rendered by imprinting an helical phase delay on the STED laser wavefront (Vortex Phase Plate VPP-1, RPC photonics, Rochester NY USA). Excitation was typically performed at $10 \mu \mathrm{W}$, with a dwell time of $20 \mu \mathrm{s}$; STED depletion was performed at $150 \mathrm{~mW}$, allowing a resolution of less than $35 \mathrm{~nm}$ (calibration not shown).

Laser focusing and collection of fluorescence was performed by the same oil immersion objective (UPlanSApo 100x 1.4 Oil, Olympus Corporation, Tokyo, Japan). Fluorescence emission 
was detected by a Single Photon Counting module (SPCM-AQRH-13-FC, Excelitas Technologies Corp., Waltham MA USA). An appropriate series of dichroic mirrors and optical filters separate the excitation and STED light from fluorescent emission. Experiments were run with the software ImSpector (MPI fr biophysikalische Chemie, Göttingen, Germany); data analysis was performed with $\mathrm{Fiji}^{7}{ }^{7}$

\subsection{Preparation of Halotag-fusion proteins}

The chemical crosslinking strategy was replaced by Halotag protein binding, described in Section 3.9. Lifeact-Halotag sequence was cloned in plasmid pET17b with a His tag at C-terminus. The engineering of the construct was done from individual protein sequences. Lifeact sequence was obtained from company (Ibidi GmbH, Martinsried, Germany) and oligos were purchased commercially (Sigma-Aldrich Chemie Gmbh, Munich, Germany). Halotag sequence was cut-out from plasmid, pFC14A (PROMEGA GMBH, Mannheim, Germany) by using suitable restriction enzymes. Sequence of His tag was added at the C-terminus of the protein sequence using suitable primers in PCR.

Post cloning, E. coli BL21(DE3) (Invitrogen, Carlsbad, CA, USA) were transformed with the pET17b plasmid and grown to an optical density of about 0.8 at $37^{\circ} \mathrm{C}$. TPM medium was used, supplemented with ampicillin $(0.1 \mathrm{mg} / \mathrm{ml})$. The medium consists of $2 \%$ tryptone, $1.5 \%$ yeast, $0.8 \% \mathrm{NaCl}, 0.2 \% \mathrm{Na}_{2} \mathrm{HPO}_{4}$ and $0.1 \% \mathrm{KH}_{2} \mathrm{PO}_{4}$.

Protein expression was induced using $0.1 \mathrm{mM}$ IPTG for $3 \mathrm{hrs}$ at $37^{\circ} \mathrm{C}$. Variations in this step were tested by adding $5 \%$ ethanol and reducing incubation temperature to $25^{\circ} \mathrm{C}$. This was done to reduce the protein expression and thereby reduce any protein aggregation due to misfolding of proteins. After induction, the cells were harvested by centrifugation and resuspended in a $20 \mathrm{mM}$ imidazole buffer, $\mathrm{pH} 7.4$, supplemented with $1 \mathrm{mM}$ each of DTT, $\mathrm{MgCl}_{2}, \mathrm{EGTA}, \mathrm{BME}$ and $150 \mathrm{mM} \mathrm{NaCl}$ before lysis using ultrasound in the presence of lysozyme and DNAsel. After separation from cell debris by centrifugation, the cytosol was incubated for $1 \mathrm{hr}$ at $4^{\circ} \mathrm{C}$ with Ni-NTA-column material (Qiagen, Hilden, Germany) and then transferred to a syringe column. After washing the column with $80 \mathrm{mM}$ imidazole and $300 \mathrm{mM} \mathrm{NaCl}$, bound protein was eluted using a $300 \mathrm{mM}$ imidazole buffer containing $10 \mathrm{mM}$ ATP and $1 \mathrm{mM}$ DTT.

\subsubsection{Protein binding assay}

A binding assay was done to verify binding of the fusion protein to actin filaments. $1 \mathrm{mg} / \mathrm{ml}$ actin was polymerized using a protocol described previously. Actin was incubated with LifeactHalotag in a molar ratio of 1:10 for $1 \mathrm{hr}$. The samples were then spun-down in an air driven ultracentrifuge (Beckman Coulter, Inc., Palo Alto, CA) at $100000 \mathrm{~g}$ for $1 \mathrm{hr}$. The supernantant was decanted into another tube and the pellet resuspended in an equivalent amount of $1 \mathrm{X}$ Polymix buffer. The samples were run on a SDS PAGE gel to check for co-sedimentation of the protein with actin. 


\subsubsection{SDS PAGE protocol}

The supernatant and pellet, obtained after centrifugation from binding assay, were run on a SDS PAGE gel. The samples were pre-treated as follows. $10 \mu \mathrm{l}$ of each sample was mixed with $10 \mu \mathrm{l}$ of $2 \mathrm{X}$ sample buffer and heated to $95^{\circ} \mathrm{C}$ for $5 \mathrm{~min}$. The sample buffer consists of 2-Mercaptoethanol, $0.1 \%$ bromophenol blue, $0.0005 \%$ glycerol, $10 \%$ SDS (electrophoresisgrade), $2 \%$ Tris- $\mathrm{HCl}, 63 \mathrm{mM}$ ( $\mathrm{pH} 6.8)$. The treated samples were run on a $10 \%$ polyacrylamide gel at $150 \mathrm{~V}$ for 60-70 min. The gel was stained with PageBlue Protein Staining Solution (Life Technologies $\mathrm{GmbH}$, Darmstadt, Germany) for approximately 30 minutes and imaged with Intas ECL Chemocam imager (INTAS Science Imaging Instruments GmbH, Sartorius, Göttingen, Germany).

\subsection{Cell culture}

The DNA force sensors were introduced into human mesenchymal stem cells using different methods, as described in Chapter 4. The maintenance of cells and the various transfection protocols are elaborated below.

Adult human mesenchymal stem cells from bone marrow [hMSCs] (Lonza Group Ltd, Basel, Switzerland, PT-2501, Lot\#2F3478) were used in all cell experiments. We seeded cells at a density of 100000 to 200000 per flask in T75 Corning cell culture flasks (Corning Incorporated Life Sciences, Tewksbury, MA, USA). The cells are kept in Dulbeccos Modified Eagle Medium, DMEM (Life Technologies GmbH, Darmstadt, Germany) $+10 \%$ FBS and $+1 \%$ penicillin/ streptomysin (Life Technologies $\mathrm{GmbH}$, Darmstadt, Germany). The cells were split every 2-3 days by detaching from substrate using $0.05 \%$ trypsin-EDTA (Life Technologies GmbH, Darmstadt, Germany) following the company specified usage instructions for human mesenchymal stem cells.

\subsubsection{Preparation of Lifeact-Halotag and DNA mixture}

Lifeact-Halo protein was purified from E.coli, as described above. The concentration of the purified protein was $0.6 \mathrm{mg} / \mathrm{ml} .1 \mu \mathrm{g}$ DNA strands, $\mathrm{H}$ and $\mathrm{F}$ with thiol ends, were annealed

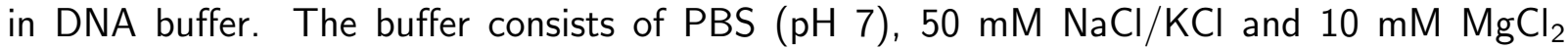
The annealed DNA was reduced with TCEP for $1 \mathrm{hr}$ and incubated with $10 \mu \mathrm{M}$ iodoacetamide functionalized Haloligand (PROMEGA GMBH, Mannheim, Germany). The DNA-ligand mixture was incubated with $10 \mu \mathrm{M}$ Lifeact-Halotag for 15 minutes before further treatments. This solution is referred to as 'Lifeact-Halotag and DNA mixture' in following subsections. 


\subsubsection{Viafect treatment}

Viafect transfection reagent was obtained commercially (PROMEGA GMBH, Mannheim, Germany). We followed company specified protocol for introducing Lifeact-Halotag and DNA mixture using Viafect into the cells. Briefly, approximately 2500 cells were plated out in a Petri dish, one day before transfection. The medium was removed and cells were washed with sterile PBS, prior to transfection. Lifeact-Halotag and DNA $(60 \mu \mathrm{l})$ was mixed with Viafect $(12 \mu \mathrm{l})$ and incubated for 15 minutes at room temperature. DMEM was then added to the mixture to make up the volume to $300 \mu \mathrm{l}$. The mixture was added to adhered cells, followed by incubation for $1 \mathrm{hr}$ at $37^{\circ} \mathrm{C}$ and $5 \% \mathrm{CO}_{2}$, prior to imaging over a period of $24 \mathrm{hrs}$.

\subsubsection{Electroporation}

The Nucleofection mixture was prepared by carefully mixing $82 \mu$ l Nucleofector Solution P1, $18 \mu \mathrm{l}$ Supplement 1 and Lifeact-Halotag and DNA mix (contained approximately $1 \mu \mathrm{g}$ DNA). Trypsinized cells were centrifuged at $1000 \mathrm{rpm}$ for 5 minutes. The cell pellet was resuspended in the prepared solution and put in Nucleocuvette. A1, P1 slot in Xunit of the 4D Nucleofector (Lonza Group Ltd, Basel, Switzerland) was used for electroporation. Pulse code FF 104 was used on the cells followed by incubation for 3-5 min at RT. Warm $1 \mathrm{ml}$ DMEM medium was added to a Petri-dish and electroporated cells were plated out (approximately 2500 cells in one dish). The plates were incubated at $37^{\circ} \mathrm{C}$ and $5 \% \mathrm{CO}_{2}$ for $1 \mathrm{hr}$ to allow recovery, followed by imaging over a period of $24 \mathrm{hrs}$.

\subsubsection{Saponine treatment}

$0.05 \mathrm{~g} / \mathrm{I}$ Saponin was prepared in a hypotonic buffer consisting of $130 \mathrm{mM}$ sucrose, $50 \mathrm{mM}$ $\mathrm{KCl}, 50 \mathrm{mM}$ potassium acetate and $20 \mathrm{mM}$ HEPES. Approximately 2500 cells were trypsinized and treated with Saponin and Lifeact-Halotag and DNA mix using the following protocol.

1. Wash cells $1 \times$ with PBS.

2. Add Saponin and Lifeact-Halotag and DNA mix and shake for $5 \mathrm{~min}$.

3. Wash with PBS.

4. Incubate in medium for at least 30-45 min.

The treated cells were allowed to recover for $1 \mathrm{hr} 37^{\circ} \mathrm{C}$ and $5 \% \mathrm{CO}_{2}$ and imaged over a period of $24 \mathrm{hrs}$. However, all the cells died after treatment with Saponin. Therefore, this method of transfection cannot be used in our experiments. 


\subsubsection{Fuse-It-P method}

Fuse-It-P (Ibidi GmbH, Martinsried, Germany) was obtained commercially as a lyophilized powder. Company specified protocol was followed thereafter, with minor modifications. The protocol used is given below.

1. Add $40 \mu \mathrm{l}$ of the Lifeact-Halotag and TMR Haloligand (no DNA) to a Fuse-It-P vial and mix thoroughly by vortexing until complete resuspension.

2. Sonicate the mixture in a standard ultrasonic bath for 5-10 minutes at room temperature or lower.

3. Fill up the fusogenic mixture with $20 \mathrm{mM}$ HEPES buffer $(\mathrm{pH} \mathrm{7.4)}$ or sterile water, to make a total volume of $100 \mu \mathrm{l}$, then vortex for 30 seconds.

4. Make 2 batches of $10 \mu \mathrm{l}$ and $25 \mu \mathrm{l}$ of the fusogenic mixture in $500 \mu \mathrm{l} 1 \mathrm{X}$ PBS by vortexing for 30 seconds.

5. Replace the culture medium of the cells (approximately 2500) with the diluted fusogenic mixture.

6. Incubate for 5 minutes at $37^{\circ} \mathrm{C}$.

7. Replace the fusogenic mixture with fresh culture medium to stop fusion.

8. After fusion, the cells were immediately transferred to microscope chamber and imaged over a period of $24 \mathrm{hrs}$.

\subsubsection{Imaging of cells}

Cells were imaged for $24 \mathrm{hrs}$, using a $32 \mathrm{X}$ objective, on a regular epifluorescence microscope, Axio Observer.Z1 (Carl Zeiss Microlmaging GmbH, Jena, Germany). The microscope is fitted with a heating System (Ibidi $\mathrm{GmbH}$, Martinsried, Germany) for maintaining temperature at $37^{\circ} \mathrm{C}$. A gas incubation system for $\mathrm{CO}_{2}$ with a humidifying column (Ibidi $\mathrm{GmbH}$, Martinsried, Germany) is also included to maintain $5 \% \mathrm{CO}_{2}$ in the chamber. Images were recorded in phase contrast as well as in fluorescence ( $\lambda_{\text {excitation }}$ and $\lambda_{\text {emission }}$ was $494 \mathrm{~nm}$ and $520 \mathrm{~nm}$ ). 


\section{Bibliography}

[1] Zadeh, J. N.; Steenberg, C. D.; Bois, J. S.; Wolfe, B. R.; Pierce, M. B.; Khan, A. R.; Dirks, R. M.; Pierce, N. A. Journal of computational chemistry 2011, 32, 170-173.

[2] Karedla, N.; Chizhik, A. I.; Gregor, I.; Chizhik, A. M.; Schulz, O.; Enderlein, J. ChemPhysChem 2014, 15, 705-711.

[3] Zhou, H. M.; Brust-Mascher, I.; Scholey, J. M. The Journal of Neuroscience 2001, 21, 3749-3755.

[4] Zimmermann, J. L.; Nicolaus, T.; Neuert, G.; Blank, K. nature protocols 2010, 5, 975-985.

[5] Yuan, C.; Chen, A.; Kolb, P.; Moy, V. T. Biochemistry 2000, 39, 10219-10223.

[6] Moy, V. T.; Florin, E.-L.; Gaub, H. E. Colloids and Surfaces A: Physicochemical and Engineering Aspects 1994, 93, 343-348.

[7] Schindelin, J. et al. Nature methods 2012, 9, 676-682. 




\section{Glossary}

MT Microtubule

DNA Deoxyribonucleic acid

ATP Adenosine triphosphate

GTP Guanosine triphosphate

PCR Polymerase chain reaction

NHS N-Hydroxysuccinimide

TMR Tetramethylrhodamine

TCEP Tris(2-carboxyethyl)phosphine

$\mathrm{SH} \quad$ Sulfhydryl

APTES (3-Aminopropyl)triethoxysilane

DETA Diethylenetriamine

NA Numerical aperture

LVE Linear viscoelastic

LAOS Large amplitude oscillatory shear

STED Stimulated emission depletion

FRET Frster/fluorescence resonance energy transfer

NEM N-Ethylmaleimide

DTT Dithiothreitol

EDTA Ethylenediaminetetraacetic acid

PCMPS p chloromercuribenzenesulfoate

DTNB 5, 5' - dithiobis(2-nitrobenzoic acid)

GMPCPP Guanosine-5-[( $\alpha, \beta)$-methyleno]triphosphate

$\mathrm{ABP} \quad$ Actin binding protein 


$\begin{array}{ll}\text { PBS } & \text { Phosphate buffered saline } \\ \text { HEPES } & \text { 4-(2-hydroxyethyl)-1-piperazineethanesulfonic acid } \\ \text { SDS } & \text { Sodium dodecyl sulfate } \\ \text { PAGE } & \text { Polyacrylamide gel electrophoresis } \\ \text { Sulfo-SMCC } & \text { Sulfosuccinimidyl 4-(N-maleimidomethyl)cyclohexane-1-carboxylate } \\ \text { IPTG } & \text { Isopropyl -D-1-thiogalactopyranoside } \\ \text { TBE } & \text { Tris-borate-EDTA } \\ \text { DMEM } & \text { Dulbeccos Modified Eagle Medium } \\ \text { hMSC } & \text { Human mesenchymal stem cells } \\ \text { IF } & \text { Intermediate filament }\end{array}$





\section{Acknowledgements}

This is definitely one of my most favorite chapters in the thesis. Although it was hard to keep it between too dry and over-the-top emotional, I enjoyed writing and reminiscing some good times. I would like to begin by thanking Prof. Christoph Schmidt and Dr. Florian Rehfeldt for supporting and guiding me in this project. I am especially thankful for the opportunities to attend numerous conferences that gave me the necessary exposure to the field of biophysics. I would also like to thank Prof. Sarah Köster for taking the role of the second reviewer and for the kind words of encouragement through the years. I would also like to thank Prof. Fred Wouters for being a part of my thesis committee and for the helpful suggestions during the committee meetings.

Next are our technicians- Kerstin von Roden, Charotte Willms, Ulrike Schulz and Tanja Gall. My work in the lab was so much easier, thanks to your efficient help and support. I am particularly grateful to Tanja for the endless gels and Ulrike for her help with cloning. I also want to thank Dr. Dieter Klopfenstein and Dr. Eugenia Butkevich for the helpful discussions and advice in troubleshooting my experiments. I have been lucky to have some great collaborations during these years. I would like to thank Dr. Jonathan Bath and Prof. Andrew Turberfield for their help in designing the DNA sensor. A special thanks to Jon, also for helping me so much during my visit to Oxford. I would like to thank Prof. Fred MacKintosh for helpful discussions regarding the rheology experiments. Misha Sheinman, it was a learning experience working with you. Your sharp mind and blunt comments shaped my perspective towards science. I also want to thank Fransesco Rocca for help with STED imaging and for his kind words of moral support.

Before going on to thank everyone with whom I have personally bonded here, I want to begin with my family and friends back home. First and foremost is my mother, Pooja Prabhune, who single-handedly got me where I am. Mom, you are my inspiration of an independent woman and I dedicate this thesis to you. Secondly, my sister, Meghana (a.k.a Guddi), is the one I resonate the most with. You always make me smile with your stories even from thousands of miles apart. Gita and Siddharth, who have always being my respite from work. You will be the first people I run to when I finish; I cherish the security of having family so close, thanks to you! Special thanks to Payal, Jui, Maithili, Rugved, who keep my school and college memories fresh with the fun conversations.

Its been a great journey in Göttingen. It is difficult to summarize the contribution of all the people who touched my life and even more difficult to pen down about those who touched my heart. Nevertheless, here is a modest attempt. I have to begin with Christina a.k.a CJ; my first friend in Göttingen and definitely one of the most naive person that I know of! I appreciate you 
babysitting me in the first few boring months that made my transition in a new lab so much easier. I would like to thank Andre Düselder for all the entertaining conversations in the lab. Benchwork definitely lost its charm after you left. Papa Chris, I enjoy your sarcastic perspective on work and life in general. It convinces me in a bad situation that if you dont care with your wise experience, then its probably not worth it! Carina, Miss dependable, it is absolutely true that the lab would fall apart without you! You are the synonym to 'help' and recently working closely with you has more than confirmed that. Next, I want to thank Miquel and Paula, who took me in the sort-of Spanish speaking clan and never treated me as an outsider. The routine weekend cooking sessions and conversations are memorable and special to me! Galina, my almost-Asian friend! Thank you for lightening my mood by being my partner in crime, for coffee and gossip sessions that make our eyes sparkle. Narain, thank you for always giving me company in the lab on weekends or late nights to reassure me that I am not the only workaholic around. Many eateries have benefited so far, thanks to our appetite! Alok, you have been a true friend, always by my side. I deeply appreciate your consistent support through the years. Abhi, I am falling short of words to express gratitude for your solidarity in the past few months. Thank you for playing the good cop-bad cop routine as needed and watching as I write, like a dedicated parent. I owe completing this thesis with a sane mind only to you!

There have been many others whom I shall definitely miss including Flo, Samane, Ulrich, Alice, Freddie, Suzanne, Daniel, Philipp, Gabrielle, Nardin, Achintya, Renata, Mitja, Jannes, Nikta, Nuria, Aishwarya.

Last, but not the least, I want to thank Hrishikesh, for never second guessing my decisions. You have been a loving boyfriend, a caring fiance, a supportive husband and most importantly, my best friend throughout this journey! I look forward to beginning a new chapter in my life as this one ends.

Thank you! 


\section{Curriculum Vitae}

\section{Personal information}

Name: Meenakshi Prabhune

Affiliation: Third Institute of Physics - Biophysics,

Georg August University, Göttingen

Email: meenakshiprabhune@gmail.com

\section{Research experience}

November 2011 - July 2015: PhD in biophysics

Supervisor: Prof. Christoph Schmidt and Dr.Florian Rehfeldt

Thesis title: High resolution stress field mapping in fiber networks and cells

Affiliation: Third Institute of Physics-Biophysics, Göttingen, Germany

\section{Project 1- Design of molecular force sensor using DNA}

\section{Skills}

Nupack software for designing DNA sensor sequence

Spectrophotometer bulk experiments

Fluorescence experiments - bulk/ single molecule

Molecular biology techniques (PCR, DNA purification, PAGE)

\section{Project 2: Rheology of in vitro cytoskeletal networks}

\section{Skills}

In vitro polymerization and handling of microtubules and actin

Crosslinking chemistry

Rheology - linear and nonlinear tests

Fluorescence microscopy 
Image analysis software (Fiji)

Molecular biology techniques (plasmid cloning, protein purification techniques, protein binding assays)

\section{Soft skills}

Troubleshooting, writing proficiency, networking, team work

\section{May 2010 - October 2011: Master thesis and internship}

Supervisor: Prof. Manfred Radmacher

Thesis title: Comparison of mechanical properties of cancer cells and normal cells using AFM Affiliation: Institute of Biophysics, Bremen, Germany

\section{Skills}

Atomic force microscopy

Software- Asylum research and IgorPro

Cell culture techniques

Writing - Master thesis, 1 review, 1 paper

\section{Undergraduate education}

Oct 2008 Nov 2010: Masters in Biochemistry and Molecular Biology Affiliation: University of Bremen, Germany Grade: 1.5

April 2008: Bachelor of Industrial Microbiology Affiliation: Abasaheb Garware College (AGC), Pune, India Grade: $77.5 \%$ with distinction

\section{Publications}

Prabhune M, Belge G, Dotzauer A, Bullerdiek J, Radmacher M: Comparison of mechanical properties of normal and malignant thyroid cells. Micron; 2012 Dec;43(12):1267-72

F. Heinemann, M. Prabhune, A. Kaufmann, M. Radmacher, Cellular and Nuclear Mechanics Studied by Atomic Force Microscopy, Imaging \& Microscopy (2010) 4, 22-24.

\section{Publications in preparation}

Review on 'Molecular force sensors in cells' for Journal of Physics D: Applied Physics. 
Technical paper titled 'Sulfo-SMCC prevents end to end annealing of taxol stabilized microtubules in vitro.'

\section{Conferences}

APS meeting, March 2015, San Antonio, Texas, USA (talk)

DPG meeting, March 2014, Dresden, Germany (talk)

Biophysical Society meeting, Feb 2014, San Fransisco, California, USA (poster)

Cell mechanics meeting, September 2013, Obergurgl, Austria (talk)

APS meeting, March 2013, Baltimore, Maryland, USA (talk)

Physics of cells meeting, September 2012, Hyeres, France (poster)

DPG meeting, March 2012, Berlin, Germany (poster)

Cell mechanics meeting, October 2011, Amsterdam, Netherlands (poster)

\section{Workshops}

Active Matter: Cytoskeleton, Cells, Tissues and Flocks, April/May 2014, KITP, Santa Barbara, California, USA Physics of cells - Advanced School on Cellular Biophysics, September 2012, Hyeres, France

\section{References}

Prof. Christoph Schmidt, University of Goettingen

christoph.schmidt@phys.uni-goettingen.de

Dr. Florian Rehfeldt, University of Goettingen

florian.rehfeldt@phys.uni-goettingen.de 
